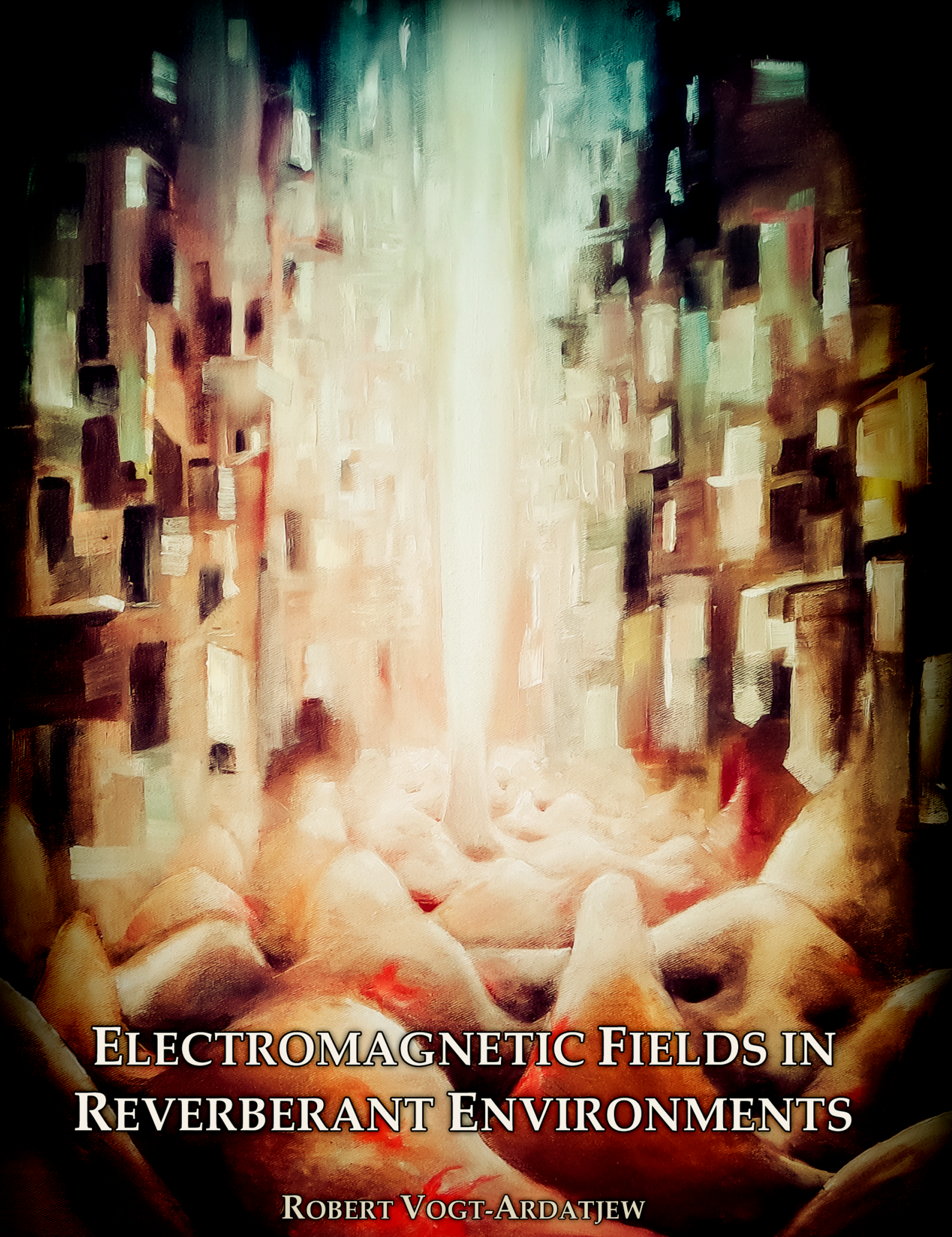




\title{
Electromagnetic Fields IN REVERBERANT ENVIRONMENTS
}

\author{
Robert Vogt-Ardatjew
}


Composition of the graduation committee:

Chairman/secretary:

Prof.dr. P.M.G. Apers

Supervisor:

Prof.dr.ir. F.B.J. Leferink

Referee:

Dr.ir. G.S. van de Beek

Members:

Prof.dr.ir. G.J.M. Krijnen

Prof.dr.ir. M.J. Bentum

Dr. R. Serra

Prof.dr. R. Zieliński

Prof.dr. C. Christopoulos

The research described in this thesis was carried out in the Telecommunication Engineering Group, which is part of the Faculty of Electrical Engineering, Mathematics and Computer Science at the University of Twente, Enschede, the Netherlands.

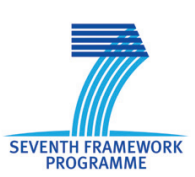

This research has received funding from the European Union's Seventh Framework Programme for research, technological development and demonstration under grant agreement No. FP7-AAT-2007-RTD-1.

CTIT

CTIT Ph.D. Thesis Series No. 17-447

Centre for Telematics and Information Technology

P.O. Box 217, 7500 AE Enschede, the Netherlands

Copyright (C) 2017 by Robert Vogt-Ardatjew

All rights reserved. No part of this book may be reproduced or transmitted, in any form or by any means, electronic or mechanical, including photocopying, microfilming, and recording, or by any information storage or retrieval system, without the prior written permission of the author.

ISBN: 978-90-365-4424-5

ISSN: $1381-3617$ (CTIT Ph.D. thesis Series No. 17-447)

DOI: $10.3990 / 1.9789036544245$

Printed by Gildeprint, The Netherlands

Typeset in $\mathrm{LAT}_{\mathrm{E}} \mathrm{X}{ }_{2} \varepsilon$ 


\title{
Electromagnetic Fields in REVERBERANT ENVIRONMENTS
}

\author{
DisSERTATION
}

to obtain

the degree of doctor at the University of Twente, on the authority of the rector magnificus, prof.dr. T.T.M. Palstra on account of the decision of the graduation committee, to be publicly defended on Wednesday 22 ${ }^{\text {nd }}$ November 2017 at 16:45h

by

Robert Andrzej Vogt-Ardatjew

born on $18^{\text {th }}$ February 1987

in Kowary, Poland 
This dissertation has been approved by: Supervisor prof.dr.ir. F.B.J. Leferink 


\section{Summary}

The phenomenon of resonating electromagnetic (EM) fields has been commonly and successfully exploited in reverberation chambers (RC) for the purpose of electromagnetic compatibility (EMC) testing, as well as modeling multipath environments. An RC is designed to maximize the amount of reflections while minimizing the losses, thus creating a statistically uniform, isotropic, and randomly polarized field. Due to the desired very high complexity of such a field, its description is best done statistically. Although largely successful, the currently used statistical models allow for a certain degree of freedom, especially with regard to defining the extreme field strengths, which are also the main cause of electromagnetic interference (EMI). Furthermore, some actual multipath, enclosed environments such as airplane fuselages, ships, or even building interiors, can unintentionally possess the ability to create enough strong reflections so that the resulting resonating EM field within them resembles the field inside a dedicated RC. Those reverberant environments (RE) can therefore utilize the methods and techniques similar to the ones used in laboratory conditions. Due to the very high complexity and diversity, resulting in increased difficulty of both numerical or analytical description of the REs, the analysis performed throughout this work is mainly experimental. Such an approach allows to bypass the often unreasonable or too generalized assumptions of the available models while utilizing purely empirical data.

The initial step made in this thesis focuses on introducing REs as environments sharing multiple aspects of EM field shaping with RCs. The experimental analysis has been performed on two example REs by means of quality factor (Q-factor), insertion loss (IL), as well as goodness of fit (GOF) tests. The results, although highly variable with frequency, indicate a possible similarity to a referential RC analyzed alongside. Furthermore, due to the difficulty of collecting large amounts of data and parameter isolation in the REs, two dedicated chambers: a classical RC and the vibrating intrinsic reverberation chamber (VIRC), have been analyzed as candidates allowing to simulate an $\mathrm{RE}$ in laboratory conditions. By the means of Q-factor, k-factor, GOF, as well as number of samples, related to field repeatability, it has been shown that the VIRC provides substantially better conditions for the given purpose. 
The search of maximum field strengths in reverberant environments has been performed by exploiting the capability of the VIRC to efficiently generate large amounts of independent samples. Performing 1-hour long measurements in the VIRC allows to obtain the desired results with very high repeatability, while giving space for parameter isolation. The first hypothesis states that the maximum-to-average (max/avg) electric field ratio depends on the losses of the environment. The measurement campaign performed to test it consisted of multiple setups with highly spread Q-factors, additionally creating a link between the laboratory and external conditions. The consistency of results indicates the rejection of the hypothesis, thus allowing to apply the same max/avg models in environments of very different $\mathrm{Q}$-factors. The second hypothesis states that the max/avg ratio depends on the properties of the receiving antenna, namely its physical size. A thorough measurement campaign using receiving monopole antennas of various lengths allowed to observe a dependency of the antenna on the distribution obtained as well as on the $\mathrm{max} / \mathrm{avg}$ ratio.

The measurement results obtained outside the laboratory conditions allowed to formulate a method of performing on-site emissions testing. Although performed in imperfect REs, a modified RC standard technique has been successfully applied, concluding that such an approach is possible and recommended. The uncertainty analysis as well as definition of requirements leads to creating a guideline of performing similar tests.

The final topic of the thesis discusses a creation of a simplified macroparameter model of field coupling to cables when neither the exact cable geometry, nor the coupling field is known. Instead of focusing on precision, this investigation aims at finding any similarities, possibly allowing to avoid the difficulties related to the very sensitive description of a non-uniform transmission line, exploiting the mixed randomness of the line geometry and multipath field excitation, as expected in real life situations. An experimental analysis performed in many configurations of the line geometry and the field excitation indicates a promising possibility of model simplification while maintaining its usability. 


\section{Samenvatting}

Het fenomeen van resonerende elektromagnetische (EM) velden is algemeen en met succes toegepast in reverberatie kamers (RC) om elektromagnetische compatibiliteit (EMC) testen uit te voeren alsook om multi-pad omgevingen te modelleren. Een RC is ontworpen om het aantal reflecties te maximaliseren terwijl de verliezen worden geminimaliseerd waardoor een statistisch uniform, isotroop en willekeurig gepolariseerd veld wordt opgewekt. Omdat het gewenste veld erg complex is, kan dit het beste statistisch worden beschreven. Hoewel grotendeels succesvol, laten de op dit moment toegepaste modellen een zekere graad van vrijheid toe, met name ten aanzien van de definitie van de extreme veldsterkten, die tevens de belangrijkste oorzaak zijn van elektromagnetische interferentie (EMI). Verder kunnen sommige feitelijke multi-pad, gesloten omgevingen zoals rompen van vliegtuigen, scheepsruimen of zelfs ruimtes in gebouwen onbedoeld zulke sterke reflecties opleveren dat de EM velden daarbinnen op de velden in een echt RC laboratorium gaan lijken. In die reverbererende omgevingen (RE) kunnen daarom methodes en technieken worden gebruikt die lijken op die in de laboratorium omgeving.

Door de zeer hoge complexiteit en diversiteit is het onmogelijk randvoorwaarden te definiëren voor zowel numerieke als analytische beschrijvingen van die RE's. Daarom worden de analyses in dit gehele werk voornamelijk experimenteel uitgevoerd. Die aanpak laat toe de de vaak onredelijke aannames van de beschikbare modellen kunnen worden omzeild terwijl puur empirische gegevens worden gebruikt.

De eerste stap in deze thesis is de introductie van RE's als omgevingen die een veelvoud van veld-opwekkings aspecten delen met $R C^{\prime}$ s. De experimentele analyse is uitgevoerd op twee voorbeeld RE's op basis van kwaliteits-factor (Q-factor), insertie-verliezen (IL) als ook "goodness of fit" (GOF) testen. De resultaten, alhoewel erg variabel over de frequenties, geven aan dat er een mogelijke overeenstemming is met een tevens onderzochte referentie-RC. Verder zijn twee specifieke testruimtes: een klassieke RC en de Vibrating Intrinsic Reverberation Chamber (VIRC) geanalyseerd als kandidaten voor de simulatie van RE's in een laboratorium omgeving. Dit omdat het erg moeilijk is om grote hoeveelheden data te verzamelen voor het isoleren van parameters 
in RE's. Door middel van Q-factor, k-factor, GOF alsook aantallen meetpunten gerelateerd aan de herhaalbaarheid van veldsituaties, is aangetoond dat de VIRC beduidend betere condities verschaft voor dit doel.

De zoektocht naar maximale veldsterktes in RE's is uitgevoerd met de VIRC omdat deze in staat is efficiënt grote hoeveelheden onafhankelijke meetpunten te genereren. Meetsessies van een uur in de VIRC laten toe de gewenste resultaten met hoge reproduceerbaarheid te verkrijgen. Tevens is er ruimte voor parameter isolatie. De eerste hypothese luidt dat de verhouding tussen de maximale en de gemiddelde elektrische veld waarden (max/avg) afhangt van de hoeveelheid verliezen in de omgeving. De meet-campagne om dit te testen bestond uit een groot aantal opstellingen met een zeer verschillende Q-factor. Dit verschafte tevens een link tussen de laboratorium en de externe condities. De consistentie van de resultaten duidt op een afwijzing van deze hypothese. Dat betekent dat de zelfde max/avg modellen kunnen worden toegepast in omgevingen met grote verschillen in Q-factor. De tweede hypothese luidt dat de max/avg verhouding afhangt van de eigenschappen van de ontvanger, met name zijn fysieke afmetingen. Een gedegen meet-campagne gebruikmakend van ontvang staaf-antennes met variërende lengte gaven aan dat de afmetingen van de antenne van invloed zijn op zowel de gemeten veldverdeling als ook op de max/avg verhouding.

De meetresultaten die buiten de laboratorium condities zijn verkregen, laten toe een test methode te definiëren om in-situ emissie metingen te doen. Alhoewel uitgevoerd in onvolkomen RE's, kan een aangepaste standaard RC techniek met succes worden toegepast. Dat geeft aan dat zo'n benadering mogelijk is en aan te bevelen. De onzekerheids-analyse alsmede de definitie van eisen leidt tot een richtlijn om vergelijkbare testen uit te voeren.

Als laatste onderwerp behandelt de thesis de opzet van een vereenvoudigd macro-parameter model van veld inkoppeling op kabels wanneer noch de exacte kabel geometrie noch de koppeling bekend is. In plaats van te focussen op nauwkeurigheid, mikt dit onderzoek op het vinden van welke overeenkomsten dan ook die mogelijk kunnen voorkomen dat een zeer nauwkeurige beschrijving moet worden gemaakt van niet-uniforme transmissie-lijnen door gebruik te maken van de statistische eigenschappen in de geometrie van deze verbindingslijnen en de multi-pad belichting door het veld zoals die in praktijksituaties kan worden verwacht. Een experimentele analyse, uitgevoerd op veel verschillende combinaties van lijn geometrie en veld-inkoppeling, wijst een veelbelovende mogelijkheid om het model te vereenvoudigen terwijl het toch goed bruikbaar blijft. 


\section{Contents}

Summary i

Samenvatting $\quad$ iii

$\begin{array}{ll}\text { Contents } & \text { v }\end{array}$

$\begin{array}{ll}\text { Acronyms } & \text { ix }\end{array}$

$\begin{array}{lll}\text { I Introduction } & \text { I }\end{array}$

1.1 Motivation ................... I

1.2 Project description ...................... 2

1.3 Thesis structure ...................... 3

2 Reverberant environments 5

2.1 Introduction ....................... 5

2.2 Random fields . . . . . . . . . . . . . . 7

2.3 Environment analysis ................... 10

2.3.1 Setup descriptions ................ 10

Laboratory: reverberation chambers . . . . . . . 10

External sites: reverberant environments . . . . . . 13

2.3.2 Critical parameters and methods . . . . . . . . . . 14

Quality factor .................. 14

Rician k-factor . . . . . . . . . . . 16

Repeatability and number of samples . . . . . . 16

Goodness of fit . . . . . . . . . . . . 19

Insertion loss ................ . . 20 
2.3.3 Reverberation chamber analysis .......... 21

2.3.4 Reverberant environment analysis . . . . . . . . 28

2.4 Summary and discussion ............... 31

3 Maximum field strengths $\quad 35$

3.1 Introduction . . . . . . . . . . . . . . 35

3.2 Maximum field measurements . . . . . . . . . . . 37

3.2.1 Time dependency .................. 38

3.2.2 Q-factor dependency ................ 40

3.2.3 Antenna dependency ... . . . . . . . . . 47

Initial tests . . . . . . . . . . . . . 48

Extended tests . . . . . . . . . . . . . 48

3.3 Summary and discussion ................... 54

4 Radiated emissions in REs $\quad 57$

4.1 Introduction . . . . . . . . . . . . 57

4.2 Site classification . . . . . . . . . . . . . 58

4.2.1 AC and ATS ..................... 58

4.2 .2 RC and STS .................. 61

4.3 Experimental STS evaluation .............. 62

4.3.1 Setup description ............... 62

4.3 .2 Results ..................... 63

4.3 .3 Error and uncertainty estimation . . . . . . . . . 64

Field uniformity . . . . . . . . . . . 66

Noise and nonlinearities . . . . . . . . . . . . . . 69

Corrected results ............. . 70

4.3 .4 Insertion loss threshold . . . . . . . . 70

4.4 Summary and discussion ............... 74

5 Field-to-cable coupling in REs $\quad 77$

5.1 Introduction . . . . . . . . . . . . . 77

5.2 Measurement setups . . . . . . . . . . . . . . . 79

5.2.1 Line geometry . . . . . . . . . . . . 79 
5.2 .2 Line excitation . . . . . . . . . . . . 79

Plane wave ................... 81

Random field .............. . 81

5.3 Experimental results ............... 8 I

5.4 Simplifications towards macro-parameters . . . . . . . . 84

5.5 Summary and discussion . . . . . . . . . . . . 87

6 Conclusions and future work $\quad 89$

$\begin{array}{ll}\text { Bibliography } & 93\end{array}$

$\begin{array}{ll}\text { List of Publications } & 101\end{array}$ 


\section{Acronyms}

A-D Anderson-Darling

AC anechoic chamber

ATS anechoic test site

CDF cumulative distribution function

CI confidence interval

CLT central limit theorem

DAQ data acquisition

DC direct current

DRG double ridge guide

EM electromagnetic

EMC electromagnetic compatibility

EMI electromagnetic interference

EMRP European metrology research programme

EUT equipment under test

GEV generalized extreme value

GOF goodness of fit

GTEM gigahertz transverse electromagnetic

HIRF-SE high intensity radiated fields - synthetic environment

i.i.d. indepedent and identically distributed

IFFT inverse fast Fourier transform

IL insertion loss 
K-S Kolmogorov-Smirnov

LOS line of sight

LPDA log-periodic dipole array

max/avg maximum-to-average ratio

OATS open area test site

PDF probability distribution function

PDP power delay profile

PEM prediction of electromagnetic fields

PI prediction interval

PIC programmable interface controller

PICA planar inverted cone antenna

PWIR plane wave integral representation

Q-factor quality factor

RBW resolution bandwidth

RC reverberation chamber

RE reverberant environment

SA spectrum analyzer

SAC semi-anechoic chamber

SNR signal-to-noise ratio

STS scattering test site

TRP total radiated power

VIRC vibrating intrinsic reverberation chamber

VNA vector network analyzer 


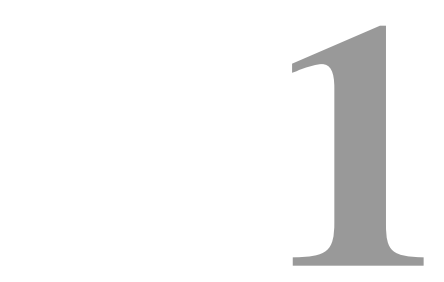

Introduction

\subsection{Motivation}

Reverberation chambers (RC) have been an object of study since their usability for the modeling of random fields was discovered [1]. They found applications in electromagnetic compatibility (EMC) for the purpose of both emissions and immunity testing of the given equipment under test (EUT). Typical RCs used in EMC are generally built in a way to mimic perfect reverberant conditions while maintaining the ability to perform tests more efficiently than the classical free space methods. A link between the theoretical models and practical results obtained in a typical $\mathrm{RC}$ has been successfully established, allowing to create a set of requirements, listed in e.g. [2]. These requirements are generally met in commercial chambers, allowing to conveniently perform EMC testing with reasonable results, as an alternative to other methods. The accepted rules and techniques are, however, optimized for commercial efficiency by simplifying the procedure and using average values or ignoring the outliers. In case of resonating random fields, this can lead to serious deviations, especially of the maximum values, which actually are the greatest electromagnetic interference (EMI) threat. RCs are also used in telecommunication engineering to create controlled multipath environments. This allowed to perform repeatable measurements with incorporated channel effects. 
The objective of the work presented in this thesis is to improve the existing models by incorporating the overlooked but critical parameters related to the extreme field strengths in reverberant environments (RE), and their influence on the fundamental EMC principles leading to EMI. As a fundamental EMC issue, this has been a topic of many studies adopting different approaches. Analytical models are very sensitive to the variation of input parameters, often based on unreasonable assumptions, or remove the crucial outliers to force a better fit. Similarly to numerical-analytical models such as those using Monte Carlo techniques. Numerical, full-wave simulations, although recently very advanced and allowing to model the setup almost perfectly, are very resource heavy and time-consuming. On the other hand, the empirical analysis, based on experiments performed in RCs is only as good as the setup used. Experiments are usually performed in setups with too limited capabilities for extensive statistical analyses, especially with regard to the amount of collected data. This method corresponds to the reality and incorporates all the possible physical parameters, even those overlooked in the other solutions. The difficulty arises from the fact that when dealing with statistics, ideally an infinite amount of samples is required to fully correspond to the mathematics, which is impossible in physical setups. However, the availability of tools such as the vibrating intrinsic reverberation chamber (VIRC) allows to push the capabilities of the empirical analysis a bit farther than previously, which became the reason to select experimental method as the primary analysis tool in this thesis.

\subsection{Project description}

The thesis focuses on the connection between the two cases mentioned in the previous section: analyzing the behavior of electromagnetic (EM) fields in conditions commonly found in reality, like airplanes, ships, even buildings, and their EMC aspects. This issue has been addressed in the High Intensity Radiated Fields - Synthetic Environment (HIRF-SE) project ${ }^{1}$ [3], which focused on creating a platform for performing numerical simulations of airplanes as a substitute for pre-testing. Therefore, such model has to be very detailed and incorporate many EMC-specific issues, e.g. shielding or coupling to cables, also in closed cavities. The latter issue belongs to the Prediction of the Electromagnetic Fields (PEM) project ${ }^{2}$ [4]. The issue of field-to-cable coupling is addressed as a simplified model to predict the worst case scenarios when

1 HIRF-SE research project has the goal to provide to the aeronautic industry a numerical modeling computer framework which can be used during the development phase, in order to ensure adequate EM performance, but also in addition and in a considerable reduction to certification/qualification testing phase.

2 The main research topic within the PEM project concerns the statistical approach on cables. 
cable geometry is unknown, usable in numerical simulations, where detailed cable tracing is impossible. It is attempted to create an extension to the models of a perfect cavity incorporating the critical parameters affecting extreme field strengths and their relations with easier measurable or calculated values, presented as simplified general rules, usable in the HIRF-SE platform. Furthermore, the imperfect environment analysis is extended towards reverberant industrial sites and the ability to perform EMC testing on-site, as a task of the European Metrology Research Programme (EMRP) ${ }^{3}$ [5].

\subsection{Thesis structure}

In Chapter 2, the principle of REs is introduced as environments possessing certain reverberant properties, e.g. capabilities of storing EM energy in form of resonances. Based on previous work, e.g. [6], it is investigated whether those environments can possibly act similarly to RCs in terms of creating statistically predictable fields or causing serious EMI threats, overly exceeding the ones expected in free space environments. This chapter also serves as the base and reasoning for the next chapters. Furthermore, two chambers are compared with the goal of simulating an imperfect RE in laboratory conditions that would allow for greater parameter isolation and manipulation, as well as an easier access to larger amounts of data.

Chapter 3 is focused on the analysis of the behavior of maximum field values in an RE under different conditions. The main objective of creating a better understanding of extreme field strength can be split into two separate, but related paths. Firstly, the creation of the extreme intensity field is analyzed for the purposes of EMC testing. Using averages requires less data and leads to results with lower uncertainty. Maximums allow for higher measurement sensitivity, although their spread is usually higher, and outliers are often ignored. In this work, the relation between maximum and average values is investigated as a function of diverse factors, allowing for simpler and more effective testing of both emissions and immunity in a laboratory environment. Secondly, the used RC is adjusted to mimic a real RE, and similar analysis is performed. Such knowledge allows to estimate the risk of an EMI threat in a given environment based only on easily measurable average values. Both approaches focus on defining a general rule linking the maximum field strength to the average, as a function of exposure time, properties of the environment or the equipment itself.

3 Improved EMC Test Methods in Industrial Environments project within EMRP focuses on the performance, characterization and improvement of the already existing alternative test methods, and then extensive correlation will be established between the existing alternative methods used in industry and the standard test methods together with the uncertainty calculations. 
The objective of Chapter 4 is to address the issues of performing on-site radiated emissions testing. The available methods are analyzed and alternative solutions are suggested. The best suited method for use in an environment exhibiting certain reverberant properties is selected and evaluated experimentally by performing measurements outside of the laboratory.

The goal discussed in Chapter 5 is to create a simplified macro-parameter model of field coupling to transmission lines when neither the exact line geometry, nor the coupling field is known. Instead of focusing on precision, the investigation aims at finding any similarities, possibly allowing to avoid the difficulties related to the very sensitive description of a non-uniform line, exploiting the mixed randomness of the line geometry and multipath field excitation, as expected in real life situations. 


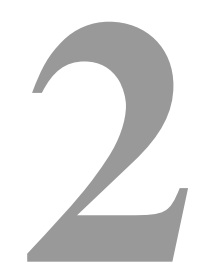

\section{Reverberant environments}

\subsection{Introduction}

In free space, only a single propagation path exists between two points, therefore the field can be analyzed in a deterministic way. If a reflection exists, the two waves interfere with each other depending on the phase, amplitude, polarization, and direction of propagation. The analytical description of such a field is only possible if all the parameters are known. However, with increasing amount of reflections, the prediction of the total field becomes very complex, even impossible if not all the critical parameters of the environment are known, which is usually the case in reality. The deterministic models become unreasonable, and statistical multipath models are used instead. Generalizing the infinite amount of critically influential solutions, such as phase or amount of superimposed rays, by using random variables allows to create a statistical model. While not able to determine the exact field properties at a given point, such a model defines a distribution of possible outcomes, depending on more general macro parameters.

As opposed to deterministic free space methods such as anechoic chambers (AC), reverberation chambers utilize the multipath behavior of the field. They are designed and built to maximize the efficiency and effectivity of EMC testing in terms of measurement time, sensitivity, repeatability, and also costs. To satisfy those requirements and provide optimal results, RCs exploit the 
property to create strong resonances, which significantly increase the amplitude of the measured field, almost entirely overpowering the non-resonating components. The isotropy, statistical uniformity, and random polarization of the bouncing waves inside a perfect RC allow the measurements of statistics, such as the average, to be performed with reasonably high repeatability. The resonances can only occur under specific conditions depending on the frequency, chamber size, and their strength varies inverse proportionally to the losses. However, if the amount of similarly excited resonances in a chamber is high enough, the statistical model becomes similar to the classical multipath model, inheriting the same distributions.

Typical ACs or RCs are validated against strict sets of requirements before they can be utilized as standardized EMC measurement facilities. As seen in Table 2.1, they usually occupy the opposite ends of the scale in terms of reflections. The exact category boundaries are defined by the adequate standards. Real environments such as an airplane fuselage, a storage hall, or a building interior do not belong to any of the aforementioned categories even though they possibly also are highly reflective. In [6], such spaces can be defined as REs if they satisfy the following requirements:

1. The space must be large in terms of the wavelength of the EM waves being considered.

2. The space must be suitably reflective.

Then, depending on the amount of reflections, in [6] generally referred to as reverberation index, they can move closer to a perfect, strongly resonating $\mathrm{RC}$, but also towards free space conditions. Moreover, opposite to validated RCs, the fields in real REs can be very unpredictable, depending on many factors. From the EMC perspective, the multipath behavior inside closed spaces might cause dynamic changes of the field strength in a resonance, possibly causing significant EMI issues. What is desired in RCs, now becomes the greatest threat. Because resonating fields are dominant over non-resonating ones even in a mildly reverberant environment, they are the main focus of this work.

In this chapter, the REs are introduced and analyzed by performing a comparison with two dedicated RCs. The critical differences and similarities are discussed through the analysis of the selected tools and methods commonly used in RC techniques. Furthermore, a side to side comparison of the two RCs is performed in order to find the optimal setup used in later chapters for modeling an imperfect RE, finding the extreme field strengths, and performing on-site EMC testing. 
Table 2.1: Simplified site classification.

\begin{tabular}{|c|c|c|}
\hline \multicolumn{2}{|c|}{$\leftarrow$ Lower $\mathbf{Q}$} & Higher $Q \rightarrow$ \\
\hline \multicolumn{2}{|c|}{ Q threshold } & Q threshold \\
\hline $\begin{array}{c}\text { Anechoic Chamber } \\
\text { (AC) }\end{array}$ & & $\begin{array}{l}\text { Reverberation } \\
\text { Chamber (RC) }\end{array}$ \\
\hline $\begin{array}{l}\text { - Fulfills the } \\
\text { requirements from } \\
\text { an AC standard. } \\
\text { - Measurement } \\
\text { procedure } \\
\text { according to the AC } \\
\text { standard. }\end{array}$ & Grey area & $\begin{array}{l}\text { - Fulfills the } \\
\text { requirements from } \\
\text { an RC standard. } \\
\text { - Measurement } \\
\text { procedure according } \\
\text { to the RC standard. }\end{array}$ \\
\hline
\end{tabular}

\subsection{Random fields}

It is said that electromagnetics are all about boundary conditions and Maxwell's equations. Using those tools, it is theoretically possible to describe the field in any given point as long as every single parameter of the environment is known. This is commonly used in numerical methods, which, with some careful modeling, can output accurate results [7]. However, without having the adequately high amount of detailed information about the environment where many reflections from undefined boundaries occur, it becomes almost impossible to have knowledge about every single wave, and a statistical approach has been proven to be more reasonable. Many multipath models exist and have found applications in telecommunication engineering for the purpose of channel modeling with fading effects [8]. It is possible to successfully apply those models in imperfectly, generally described, changing environments, with the necessity to provide only some basic, empirically obtained information, e.g. distribution scaling parameters such as the average received power.

The principle of most statistical models is the central limit theorem (CLT). It implies that a sum of many independent and identically distributed (i.i.d.) random variables tends to become a normal distribution [8]. The electric/magnetic field can be described with six parameters: complex numbers having a real and imaginary component in three orthogonal directions. In terms of perfectly random EM fields, it can be shown that [9]:

- the total electric field magnitude $\left|E_{T}\right|$ comes from a Chi distribution with 6 degrees of freedom

$$
f\left(\left|E_{T}\right|\right)=\frac{\left|E_{T}\right|^{5}}{8 \sigma^{6}} e^{-\frac{\left|E_{T}\right|^{2}}{2 \sigma^{2}}}
$$


- the electric field magnitude in a single direction $\left|E_{x}\right|$ comes from a Chi distribution with 2 degrees of freedom, known as Rayleigh distribution

$$
f\left(\left|E_{x}\right|\right)=\frac{\left|E_{x}\right|}{\sigma^{2}} e^{-\frac{\left|E_{x}\right|^{2}}{2 \sigma^{2}}}
$$

- the total power, proportional to $\left|E_{T}\right|^{2}$, comes from a Chi-square distribution with 6 degrees of freedom

$$
f\left(\left|E_{T}\right|^{2}\right)=\frac{\left|E_{T}\right|^{4}}{16 \sigma^{6}} e^{-\frac{\left|E_{T}\right|^{2}}{2 \sigma^{2}}}
$$

- the power in a single direction, proportional to $\left|E_{x}\right|^{2}$, comes from a Chisquare distribution with 2 degrees of freedom, known as exponential distribution

$$
f\left(\left|E_{x}\right|^{2}\right)=\frac{1}{2 \sigma^{2}} e^{-\frac{\left|E_{x}\right|^{2}}{2 \sigma^{2}}}
$$

In all cases, $\sigma^{2}$ is the variance of each of the six field components.

In electrically large, closed environments where many reflections are present, resonating fields are created. A continuous stream of EM energy trapped between reflectors adds to itself, creating a standing wave [10]. The field strength in anti-nodes is generally significantly higher than the amplitude of a non-resonating field. Therefore, to satisfy the CLT requirements regarding the identical distributions of added random variables, only the resonating fields are taken into account, implying a strictly modal analysis. Depending on the geometrical properties, i.e. boundary conditions, a chamber with many reflectors allows different possible standing wave solutions, i.e. modes. Some of these modes can be excited and play a dominant role in the creation of the total field. The amount of excited modes and their spacing in frequency is governed by the properties of the chamber. Additionally, each mode, being a resonance, has a given quality factor (Q-factor), therefore occupies a certain bandwidth [10]. In a large chamber, multiple modes of higher order can overlap in a single point in space [11], satisfying the fundamental requirement of the CLT, and inheriting the statistical properties of the classical multipath models, which results in similar distribution functions.

RCs are designed to create random resonating fields, satisfying the CLT in a large and continuous range of frequencies, while maximizing the obtainable field strengths. An ideal RC environment is statistically uniform, isotropic, and randomly polarized [2]. A typical RC is a highly conductive enclosure, a Faraday cage, which ensures high composite Q-factor, therefore strong resonances. Being electrically large, it allows the creation of many high order 
modes. Additionally, the complexity of the boundary conditions enforces asymmetry and diverse scattering, further increasing the randomness of both angle and polarization, and minimizing the mode degeneracy [12]. In such an environment, a statistical analysis is preferred to extract any information about the measured fields in terms of emissions or immunity. A single measurement is then merely a sample coming from a whole distribution. Therefore, collection of many uncorrelated samples is necessary, and their amount directly influences the accuracy of the statistical analysis of the measured values, i.e. the uncertainties of the estimations, e.g. estimation of the mean electric field.

The collection of independent samples is performed by introducing a change in the setup that is large enough to make separate measurements uncorrelated. The most common techniques are:

- Mechanical stirring - changing the modal structure of the field by altering the boundary conditions, e.g. using a mechanical stirrer or moving walls.

- Source stirring - placing the EM source in a different point in space, therefore exciting different modes.

- Volume sampling - collecting measurements from various points in space.

- Frequency stirring - exciting different modes by altering the input signal frequency.

The RC techniques have been studied for a very long time now and the combination of analytical and experimental approaches led to the creation of a standardized and simplified set of requirements, ensuring a reasonably proper operation of a typical RC for industrial EMC purposes [2]. Due to strong isolation of the field inside a Faraday cage, along with full control over the RC interior design (volume, materials, stirrer), the overall performance is rather predictable, and easily validated. However, generally, not much is known about REs that are not RCs. REs cannot be expected to create statistically uniform, isotropic, and randomly polarized fields. Strong resonances can occur but the Q-factors of independent modes can vary due to presence of reflectors as well as apertures or concentrated losses. The CLT criterium regarding identical distributions of added random variables is under question. On the other hand, as opposed to RCs clearly undermoded due to geometrical constraints, REs such as buildings, halls, ships are usually electrically large. The amount of excited modes could be high enough to allow comparison with RCs. The influence of strong resonances could be offset by the presence of the weak ones when considering the average values. However, the prediction of the field maximums and their relation with the easily measured averages is the key interest for EMI threat analysis. 


\subsection{Environment analysis}

The initial goal necessary to fulfill the objectives addressed in this thesis is to find a link between a dedicated RC and an imperfect RE. Such a connection, if possible, would allow to apply the well established RC techniques in external environments, as well as enable modeling an imperfect environment in laboratory conditions. Both outcomes are potentially very beneficial in terms of understanding and dealing with unknown environments, as well as on-site EMC and EMI analysis. However, even more importantly, the difficulty of creating and validating empirical models in difficult external conditions could be overcome by working with more general RC models created in an isolated laboratory environment.

In this section, the environments of imperfect REs and dedicated RCs are analyzed in search of similarities, as well as potentially influential differences. Moreover, two different RCs are confronted for the purpose of finding the optimal laboratory setup used for simulating an imperfect RE, as well as creating empirical models.

The following environment type sets are analyzed:

1. Laboratory: a classical RC with a single mechanical mode stirrer compared with the VIRC.

2. External sites: two examples of different REs, an office and an industrial workshop, compared additionally with a referential, standard RC (other than the previous).

\subsubsection{Setup descriptions}

The analyzed environment types consist of two RCs and two examples of an $\mathrm{RE}$ (plus an RC reference). Because the RC and the VIRC utilize different stirring techniques, they excel in different applications. On the other hand, the two selected REs, an office and an industrial workshop, are considered as two examples of the same general environment type, and the same methods are applied in both. Additionally, the latter REs are studied along a standard $\mathrm{RC}$, also using the same tools, allowing for an even more direct comparison.

\section{Laboratory: reverberation chambers}

The first analyzed chamber is a classical RC with a single mechanical mode stirrer. These kind of chambers are commercially available and commonly used for EMC testing [2]. The analyzed chamber is shown in Figure 2.1. It is a $150 \mathrm{~cm} \times 130 \mathrm{~cm} \times 100 \mathrm{~cm}$ Faraday cage with a $60 \mathrm{~cm} \times 60 \mathrm{~cm}$ frontal 
opening sealable with a hatch. As seen in Figure 2.2, inside the chamber there is a typical vertical Z-folded mode stirrer with $40 \mathrm{~cm}$ in diameter located in the back, outside of the symmetry planes of the box. It is rotated using a stepper motor controlled with a computer, therefore allowing to use both mode stirring and mode tuning techniques with very high resolution $\left(0.007^{\circ}\right.$ with microstepping). A horizontally polarized, directional log-periodic dipole array (LPDA) antenna was used for transmitting the energy directly at the stirrer, ensuring the maximum stirring efficiency by minimizing the unstirred components [13]. A receiving discone antenna [14] had a vertical polarization, minimizing the line of sight (LOS) component.

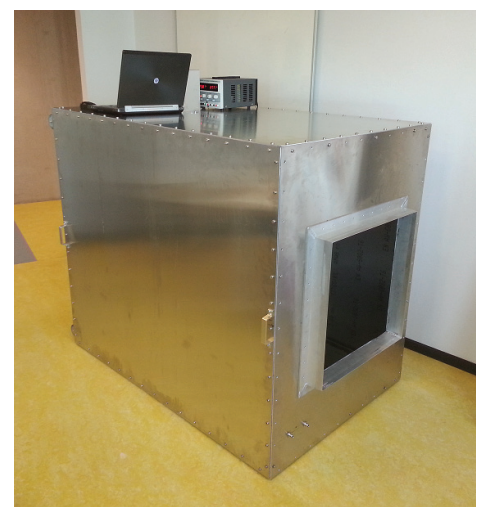

Figure 2.1: External view of the classical RC.

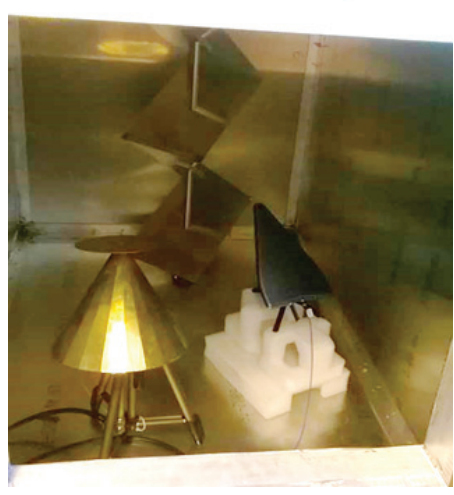

Figure 2.2: Equipment layout inside the RC.

The second analyzed chamber, the VIRC, similar to the one presented in [15], is shown in Figure 2.3. Its dimensions are $150 \mathrm{~cm} \times 120 \mathrm{~cm} \times 100 \mathrm{~cm}$, making it only slightly smaller than the used RC. This difference is compensated by the lack of a mode stirrer inside. The mode stirring is performed by introducing local changes on the surface of the chamber walls, which are made of a flexible, but highly conductive fabric. Such a motion is implemented by two DC motors, which pull the fabric from two sides. Additionally, apart from having slightly different frequencies, one of the motors changes its direction of rotation in a pseudo-random manner. Because of these factors combined with the very complex and unpredictable behavior of the flexible material, the VIRC is expected to provide a significantly higher amount of independent positions than the classical RC. However, because the shaking motion is a continuous process, the VIRC can only operate in mode stirring mode. The same antennas were used as in the RC case. As seen in Figure 2.4, the directional transmitting LPDA antenna was aimed at the shaking wall, maintaining the same optimization regarding the stirring efficiency and amount of LOS components, as in the RC case. 


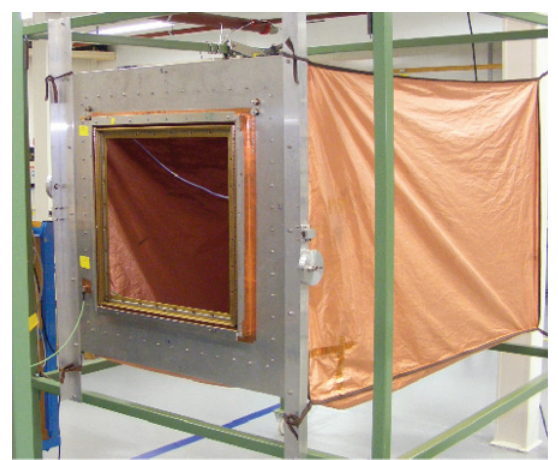

Figure 2.3: External view of the VIRC.

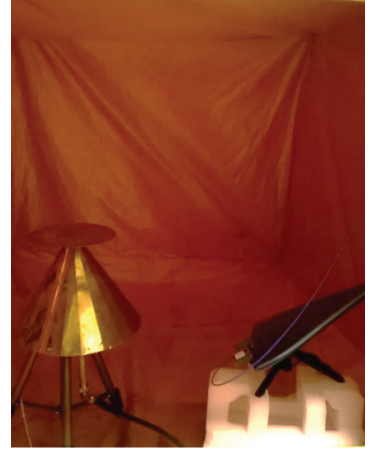

Figure 2.4: Equipment layout inside the VIRC.

For the best, direct comparison, it is desired that the same methods are used in both chambers. Because the VIRC can only operate in mode stirring mode, it became the selected technique for the RC as well. To maximize the accuracy of this analysis, it is important to record subsequent samples over time with high sampling rate. Unlike in $\mathrm{RC}$, where the rotation speed of the stirrer can be freely controlled, the stirring efficiency of the VIRC is optimal for fast and dynamic shaking of the flexible material. At the same time, it is desired to measure both magnitude and phase of the received signal. Therefore, for complex data measurements in the time domain, a USRP $-2920^{1}$ was used. The data streams were recorded using LabVIEW software in the nine main frequency steps: $400 \mathrm{MHz}, 500 \mathrm{MHz}, 600 \mathrm{MHz}, 700 \mathrm{MHz}, 800 \mathrm{MHz}, 900$ $\mathrm{MHz}, 1 \mathrm{GHz}, 1.5 \mathrm{GHz}, 2 \mathrm{GHz}$. The selected range covers the frequencies between the lowest usable frequency (LUF) of both the RC [16] and the VIRC [17], and the highly overmoded region, where both chambers operate very effectively. The continuous streams of data were recorded for each frequency step independently, sampling every $1 \mathrm{~ms}$. To maximize the amount of usable data, three stirring techniques were applied:

1. Mechanical stirring

- in RC: collecting $2.5 \mathrm{k}$ samples over $360^{\circ}$ stirrer rotation.

- in VIRC: collecting $120 \mathrm{k}$ samples over 120 seconds.

2. Volume sampling: placing the receiving antenna in eight different spatial positions inside each chamber.

3. Frequency stirring: collecting samples $-10 \mathrm{MHz},-5 \mathrm{MHz}, \mathrm{o} \mathrm{MHz}, 5 \mathrm{MHz}$, and $10 \mathrm{MHz}$ from the main frequency point.

1 The National Instruments USRP-2920 is a tunable RF transceiver with a high-speed analog-todigital converter and digital-to-analog converter for streaming baseband I and Q signals. 
Additionally, all of the aforementioned measurements were carried out in loaded conditions by inserting a single pyramid of a pyramidal carbon foam absorber to create an overlap between the two chambers in terms of possible differences in volume and inherent wall losses, as well as to address the PDF skew issue addressed in Chapter 3.

\section{External sites: reverberant environments}

The analyzed REs represent two examples of environments commonly seen in the industrial setting. They were purposely selected as two cases nearing the outliers of expected spectrum of REs, with respect to their electromagnetic properties. The office environment is shown in Figure 2.5. It is a wide corridor in a semi-open area with many typical rooms with windows and doors, therefore it is expected to have poor reverberant properties. On the other hand, the workshop environment, shown in Figure 2.6, is a closed space with many metallic objects, such as cabinets, tools, machines, located on site, allowing a higher amount of reflections. Due to the high amount of apertures (e.g. windows, doors), variable wall materials (e.g. concrete, glass), hidden reflectors (e.g. pipes, cables), the total volumes are not known.

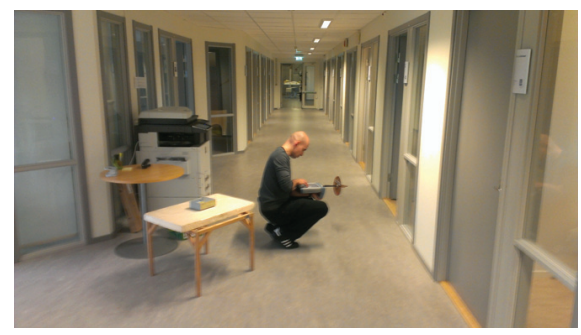

Figure 2.5: Office environment.

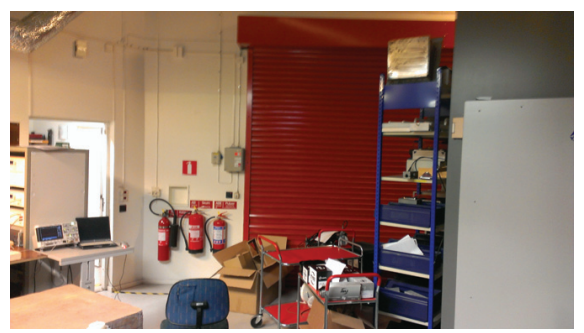

Figure 2.6: Workshop environment.

In both cases, the same setup and measurement techniques were used. Due to the lack of a mechanical stirrer available on the two sites (although portable stirrers could be a reasonable solution), the volume sampling technique was applied. The transmitting discone antenna was placed in a single position and not moved throughout the measurement process. A planar inverted cone antenna (PICA) was used for sampling the volume in the radius of circa $3 \mathrm{~m}$ around the transmitter, in 50 spatial locations. Both antennas were connected to a vector network analyzer (VNA), which was performing frequency sweeps between $300 \mathrm{MHz}$ and 1.3 GHz, giving 20001 frequency points per location. The direct coupling between the antennas was minimized by using perpendicular polarizations, as well as exploiting the minimum in the radiation pattern of the PICA by orienting it away from the discone. The measurements were also performed in a classical $2.5 \mathrm{~m} \times 2.5 \mathrm{~m} \times 3.1 \mathrm{~m}$ 
RC with the LUF around $300 \mathrm{MHz}$. The same equipment and techniques were used, with the exception of combining volume sampling (three spatial positions) with mechanical stirring.

\subsubsection{Critical parameters and methods}

In order to analyze the differences and similarities between imperfect REs and dedicated RCs, they would ideally have to be compared side by side, using the very same tools. However, due to exclusive availability of some of the methods to the laboratory RCs, as well as preferences of techniques in external REs, different approaches have to be used. In this subsection, the applied methods and critical parameters are discussed. The differences between the applied methods are shown, and reasoning for their selection under the given conditions is explained.

The discussed topics include:

- Q-factor,

- Rician k-factor,

- Number of samples,

- Goodness of fit test,

- Insertion loss.

\section{Quality factor}

The Q-factor relates the energy stored in a resonance to the energy lost per cycle of the damped oscillation [10]. A resonance with a high Q-factor allows to store more energy, thus increasing the amplitude of the resonance at the cost of limited bandwidth of excited frequencies. In an RC, every mode has its own Q-factor. The chamber can be overmoded if the bands of many neighboring modes overlap [1I]. In a properly operating $R C$, such phenomenon is desired over a continuous range of frequencies. Therefore, both upper and lower limit of Q-factors exist to ensure a proper reverberation. RCs are generally designed to operate with strong but narrow resonances to maximally increase measurement sensitivity. This is only possible above the LUF, where the mode density becomes high enough. In case of imperfect REs, where the losses are expected to be high, the bands of resonances are wide, therefore the overlap of even sparsely distanced modes is still possible, satisfying the aforementioned requirement. However, too low Q-factor can limit the reverberant properties of the environment and thus lower the ability to create high field strength resonances. Further issues related to the Q-factor non-uniformity are addressed in the next section. 
It is possible to measure the Q-factor of a single, non-overlapping mode by finding its bandwidth [10], e.g. from the transfer function measured over a frequency sweep. In case of modal overlap, it is more practical to work with average composite Q-factor, defined as the harmonic sum of individual Q-factors. In [18], the composite Q-factor (from here referred to as the Q-factor of the environment unless stated otherwise) can be calculated using

$$
Q=\frac{16 \pi^{2} V\left\langle P_{r}\right\rangle}{\lambda^{3} P_{t}}
$$

where:

$Q$ - quality factor

$V$ - environment volume

$\left\langle P_{r}\right\rangle$ - power received averaged over many field states

$P_{t}$ - power transmitted.

In case of poorly described REs, the volume is usually unknown, therefore alternative methods are preferred. In [19] it is shown that the Q-factor can be extracted from the power delay profile (PDP) without the necessity of providing information about the environment. The relation is given by

$$
\operatorname{PDP}(t)=P_{0} e^{-\omega t Q}
$$

where $P_{0}$ is the initial power level and $\omega$ is the angular frequency. The PDP is calculated from the impulse response averaged over many stirrer positions or spatial locations. It can be measured directly in time domain [20], or by performing the inverse fast Fourier transform (IFFT) on the transfer function measured in a given frequency band. The former method requires an appropriately fast equipment (RF pulse generator and detector) but is usable in any conditions, including a continuously shaking VIRC, where the measurement is much faster than the rate of change of the field. Performing measurements in the frequency domain, e.g. by using a VNA, is more easily implementable but requires the steadiness of the environment for the duration of the sweep.

In the isotropic field of an $\mathrm{RC}$, many strong resonances occur and there are many modes with similarly high Q-factors, although even in a perfect chamber the distribution of the individual Q-factors is not uniform [21]. Most significant modes are affected by the present loss mechanism, which is generally distributed uniformly on the chamber walls. Therefore, the composite Q-factor is a reasonable representation of the overall losses. In imperfect REs, the losses are concentrated around apertures or other objects. Creation of stronger and weaker resonances is hypothetically possible. As much as the average losses can be compared using the composite Q-factor, higher variation 
of individual Q-factors is to be expected, possibly leading to higher variations in the outlying statistics such as unexpectedly high maximums.

\section{Rician k-factor}

The theory behind the field distribution models inside a perfectly reverberant environment assumes that all of the summed values are i.i.d. random variables [9]. In such a case, both real and imaginary parts of the complex number describing the total field in the point of summation, according to the CLT, belong to Gaussian distributions with zero mean, i.e. they occupy the area around the origin of a scatter plot. In case of imperfections such as coupling between antennas or improper stirring, another component is added as well. It is deterministic due to being unaffected by the mechanisms introducing randomness, e.g. not reflected from the mode stirrer. When unchanged during the process of collecting the data, it causes a shift of the means of the Gaussian distribution away from the origin. Therefore, the magnitude of the created field no longer belongs to the Rayleigh, but to the Rice distribution [22], described as

$$
f\left(\left|E_{x}\right|\right)=\frac{\left|E_{x}\right|}{\sigma^{2}} I_{0}\left(\frac{\left|E_{x}\right|\left|E_{d}\right|}{\sigma^{2}}\right) e^{\left(-\frac{\left|E_{x}\right|^{2}+\left|E_{d}\right|^{2}}{2 \sigma^{2}}\right)}
$$

where $I_{0}$ is the modified Bessel function of the first kind with order zero, $\left|E_{x}\right|$ is the stirred field component, and $\left|E_{d}\right|$ is the constant, deterministic field component.

The ratio between $\left|E_{d}\right|$ and variance of the stirred field $\sigma^{2}$ is conventionally described as the Rician k-factor, defined as

$$
k=\frac{\left|E_{d}\right|^{2}}{2 \sigma^{2}}
$$

If $k=\infty$, the field becomes entirely deterministic like in typical LOS setups such as a perfect anechoic chamber. On the other hand, if $k=0$, distribution 2.7 becomes Rayleigh. For EMC testing in RCs, it is generally desired to minimize the k-factor as its dependence on the usually unknown EUT directivity impairs the measurement accuracy. However, in other applications, such as telecommunications, the ability to control the balance between the LOS component and scattered components causing fading is highly beneficial [22].

\section{Repeatability and number of samples}

Repeatability is a crucial aspect in all kinds of measurements. By having control over every significantly influential parameter of the setup, it should 
be possible to reproduce the previously obtained values. This is generally possible in systems depending mostly on deterministic relations. In such cases, the uncontrolled parameters, usually modeled with random variables, are considered as measurement uncertainties. However, in systems such as RCs, which are purely based on the statistical methods, two kinds of repeatability have to be considered.

1. The sample repeatability defines the ability to obtain the same results at every stage of data collection, e.g. for every stirrer position, even if later used for statistical analysis; a scenario when the correlation of two sequences equals 1 . It enables the analysis of each recorded sample independently, which becomes necessary for comparisons with numerical models or tracing down artifacts or outliers.

2. The statistical repeatability is defined here as the ability to obtain similar results that are calculated from statistics of the obtained data, i.e. the accuracy of the estimations. Even if the data are not entirely correlated to the previous iteration of the experiment, the estimations of their statistics such as the average or variance can be the same. If there is a reasonable cause for this to happen, the measurement is statistically repeatable. The statistical repeatability is directly related to the number of i.i.d. samples.

Reverberant environments posses the capabilities to modify the injected signal to become a random variable at the receiver level. However, due to physical constraints commonly met in a typical transmitter-receiver setup, the field can be monitored only in a single position, under the given conditions, at the given time. As such, a single measurement sample is created. For the purpose of statistical processing, larger amounts of data are necessary. Furthermore, their amount directly affects the measurement accuracy. The quality of the data is also of a huge concern - to resemble the perfect, theoretical models, the samples would ideally have to be i.i.d., although in reality, lack of correlation is a sufficient demand. In order to obtain an uncorrelated sample, an appropriately significant change in the setup has to be introduced by the means described previously in Section 2.2: mechanical stirring, source stirring, volume sampling, or frequency stirring. The applications of the 3 latter techniques are not entirely dependent on the environment itself, but are rather governed by their inherent rules regarding spatial [23] or frequency distancing [18]. Although they allow to multiply the amount of samples under specific conditions, they all bear a common flaw of not always being usable in practical applications with respect to measurement time, setup complexity, and generality. Therefore, they are often utilized as secondary methods for data multiplication. On the other hand, the former, mechanical stirring method, is 
directly related to the performance of the chamber. It also allows to obtain uncorrelated data within a more reasonable time and simpler setup (versus volume sampling and source stirring), independently of the tested equipment (unlike frequency stirring). Therefore, the capabilities of this method are analyzed in this section as performance indicators of the RC and the VIRC in the laboratory conditions. A similar analysis could not be performed in the REs because no stirrer was present at the external sites, where only volume sampling and frequency stirring were applied.

The fundamental difference between the RC and the VIRC lies in the way the mechanical stirring is applied. The RC utilizes a fully controllable mode stirrer, enabling the use of two operation techniques. Mode tuning is a technique of moving the stirrer in discrete steps, and performing the measurement, usually a frequency sweep, while it is stopped. Then, the stirrer is moved to the next defined position and the measurement is repeated. During mode stirring, the stirrer is rotated continuously while the measurement is performed at a given, single frequency point. In both cases, the samples can only be collected within a single stirrer rotation, and their correlation is dependent on the rotation angle between the subsequent positions. In the VIRC, only mode stirring is possible. Similarly to the $\mathrm{RC}$, the movement is introduced by a rotating motor. In this case however, the motor is connected to an arm pulling the flexible material. Due to the very unpredictable movement of the flexible walls, and extremely high sensitivity to any movement, new, uncorrelated positions are created even after the initial rotation. For the same reasons, mode tuning is difficult to apply.

Sample uncorrelation is generally necessary to match empirical data to their theoretical models because in the latter ones, sample independence is assumed. Using only uncorrelated data ensures maximum predictability of the measured statistics, such as the commonly used average. Therefore, in order to optimize the measurement for time efficiency, repeatability, and accuracy of estimations, only the i.i.d. samples are recorded. However, if the data consist of many samples and the model estimations are reasonably high, sample dependence does not necessary impair the matching [24]. For example, in an extreme case when even the whole dataset is repeated, therefore entirely correlated, the overall average value does not change. All of the samples come from the same distribution. On the other hand, it might even be the case that a presumingly correlated sample might be the recorded maximum, yielding additional information, that could be otherwise missed. Oversampling, although more time-consuming and resource-demanding, might be desired in certain cases. The amount of uncorrelated samples is still a very good indicator of the overall performance of an RC. It has been a subject of many studies and, as such, plenty of interesting methods and approaches are available in the literature: 
- Autocorrelation Function (ACF) method [2].

The principle of the ACF method lies in the analysis of the autocorrelation coefficient as a function of the introduced change, e.g. stirrer rotation angle.

- Stirrer Volume method [25].

This method allows to find the amount of uncorrelated samples from the volume of the stirrer inside an RC. Although it provides reasonable and easy to obtain results, it serves more as a rule of the thumb for classical RCs rather than a reliable tool due to the empirically obtained coefficients, as well as unusability in the VIRC, where no classical stirrer is present.

- Sample Difference method [26].

The total amount of uncorrelated samples can be extracted from the relative changes of the oversampled sequence of data. The strong asset of this method is that it can be used in any conditions, as it requires only the raw set of samples without any specific requirements.

- Central Limit Theorem method [27].

According to the CLT, the standard deviation of the distribution of the means measured in many positions throughout the chamber is proportional to the amount of independent samples.

- General Method [28].

Pairs of uncorrelated positions can be found by analyzing the correlation coefficients between two matrices of data measured in many different spatial positions. Because inter-sample relations are analyzed, sample repeatability is necessary.

\section{Goodness of fit}

A goodness of fit (GOF) test is a tool for performing a check whether the data comes from the tested, presumed distribution, i.e. whether the theoretical models are valid for the given setup. Because the data distributions can be affected by many, often untraceable factors, the GOF test is usually considered to be a top level performance indicator, although used as a tool for the analysis of individual parameters as well [29][30]. 
The most common tests applied in RC techniques are [24]:

1. Kolmogorov-Smirnov (K-S) test. A rather simple and robust test focusing mostly on the around-average region of the distribution. The K-S statistic is the maximum difference between an empirical and theoretical cumulative distribution functions (CDFs). The statistic is independent of the amount of samples, although it is compared to the critical value, which depends on it. According to [24], this test is rather sensitive to data correlation. The parameters of the tested distribution cannot be extracted from the data itself, but have to be known beforehand or obtained from simulations.

2. Anderson-Darling (A-D) test is based on and therefore very similar to K-S, modified by an added weighting function, which increases its power around the tails of the distribution.

3. Chi-squared $\left(\chi^{2}\right)$ test is performed on binned distributions of both theoretical and empirical data. Therefore, the impact of variable sample sizes is theoretically minimized. Although sensitive to the arbitrary selection of the bin sizes, this test is less sensitive to correlation [24], therefore potentially more usable for oversampled data. The parameters of the tested distribution can be extracted from the analyzed data, making this test particularly useful and easy to implement. Due to the mentioned advantages, this test was used throughout the thesis.

\section{Insertion loss}

The Insertion loss (IL), being the simplest of the mentioned tools, quantifies the total site losses, therefore also carries information about the site reflections. The IL is simply the amount of attenuation introduced to the channel after placing the measurement setup in a given environment. Therefore, the minimum $\mathrm{IL}_{\min }$ and average $\mathrm{IL}_{\mathrm{avg}}$ insertion losses can be calculated directly from the $S_{21}$ data, corrected for both antennas, according to equations 2.9 and 2.10, depending on whether maximum or averaged values are used, respectively. The values $\eta_{T x}$ and $\eta_{R x}$ represent total efficiencies of the transmitting and receiving antennas. These values were also used later for the calculation of the total radiated power (TRP) in Chapter 4.

$$
\begin{gathered}
\mathrm{IL}_{\min }=\frac{\eta_{T x} \eta_{R x}}{\max \left(\left|S_{21}\right|^{2}\right)} \\
\mathrm{IL}_{\mathrm{avg}}=\frac{\eta_{T x} \eta_{R x}}{\left\langle\left|S_{21}\right|^{2}\right\rangle}
\end{gathered}
$$




\subsubsection{Reverberation chamber analysis}

In this subsection, the properties of dedicated RCs are analyzed in laboratory conditions. Such information provides a reference for the RE analysis presented in the next subsection. A comparison allows to better understand the behavior of the latter, as well as decide whether the dedicated RCs could serve as a laboratory setup to mimic them. Furthermore, it is decided which of the two chambers, the classical rigid RC or the VIRC, is more suitable for this purpose, as well as other objectives of this work, e.g. extreme field strength investigation in Chapter 3 or field-to-cable coupling in Chapter 5 .

In both classical RC and the VIRC, the data were collected in a similar manner, according to the description in Section 2.3.1, with the sole difference lying in the mechanical stirring technique. The combined application of frequency stirring (five points) and volume sampling (eight position) results in 40 datasets per main frequency point. Unless stated otherwise, the results shown in this subsection represent the averages of the 40 calculated results. Such representation of the data, although neglects possible outliers e.g. due to field uniformity issues, gives a general impression of the behavior of each of the given setups in a straightforward and easy to read manner.

The assumption of similarity regarding the volumes of the classical RC and the VIRC can be validated by looking at the corresponding average, composite Q-factors, calculated from the PDP, as described in [20]. As seen in Figure 2.7, the results of both chambers are similar up to $1 \mathrm{GHz}$, and the consistency of Q-factors, also when lowered due to the loading, is maintained, implying that both chambers are indeed comparable. Beyond $1 \mathrm{GHz}$, an increasing deviation is visible, especially in the case of empty chambers where the effect of wall losses is dominant, due to higher shielding effectiveness of the thick walls of the RC as opposed to the thin flexible material of the VIRC.

Even though the layout of the antennas during the measurement was optimized to minimize the LOS component by utilizing minimums in the antenna patterns as well as cross-polarization, the complete elimination of the deterministic component is rarely possible. The Rician k-factor has been analyzed under the hypothesis that the VIRC allows for a lower k-factor due to the minimization of the deterministic reflections from the moving walls, as opposed to immovable walls of the RC. However, the results of the k-factor calculated according to [22], shown in Figure 2.8 imply that both chambers offer similar capabilities of eliminating the LOS component. The highly increased k-factor below $600 \mathrm{MHz}$ was likely caused by using a presumably directional LPDA slightly below its nominal frequency range. This is proven by the consistency between empty and loaded chamber cases, where only the scattered energy is affected by the introduced losses [13]. 


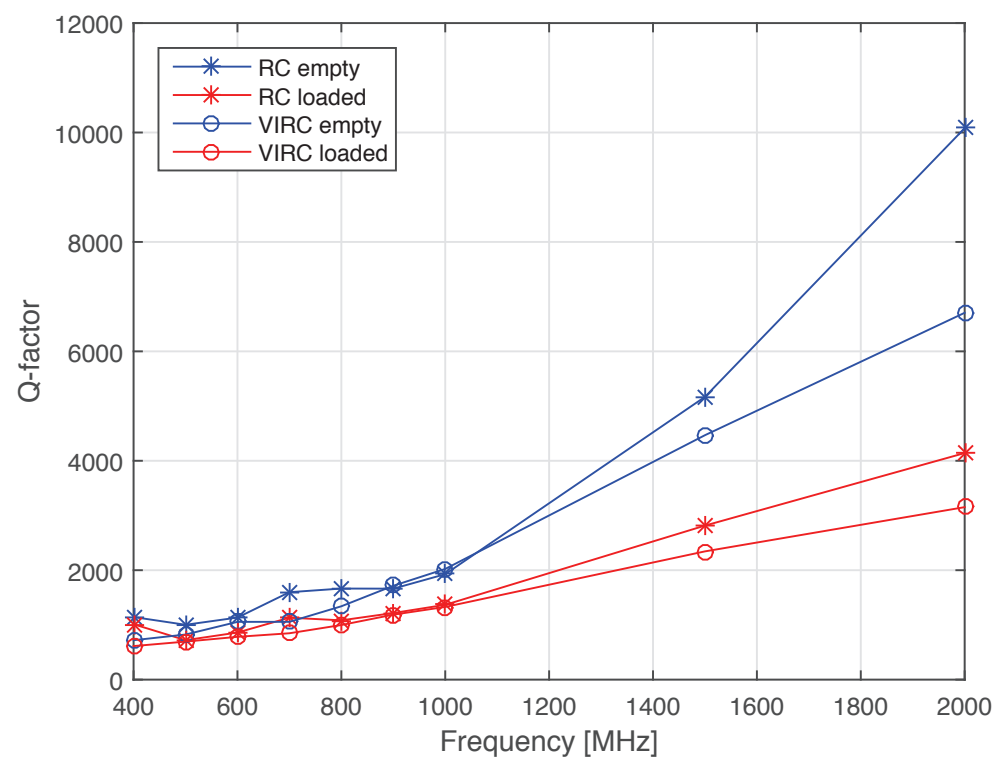

Figure 2.7: Average Q-factors of the RC and VIRC.

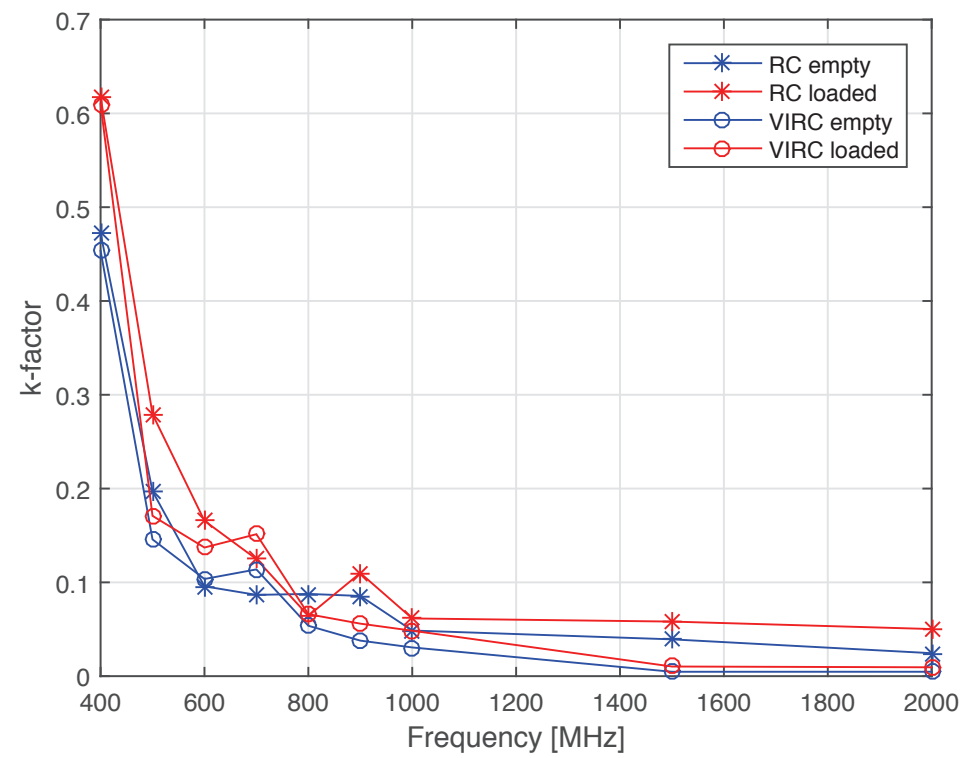

Figure 2.8: Average k-factors of the RC and VIRC. 
The topic of repeatability can be split into separate analyses of sample repeatability by means of autocorrelation function behavior, and statistical repeatability by means of spread of average and maximum values, i.e. field uniformity, directly related to the number of uncorrelated samples.

The autocorrelation function shown in Figure 2.9 returns to 1 after a $360^{\circ}$ rotation of the stirrer because the sequence of the samples is repeated in the very same order. The field strength values are also bound to the stirrer position, i.e. can easily be reacquired after moving the stirrer to an adequate position. In practice, this sample repeatability can be exploited for performing multipoint measurements by moving a single, assumingly transparent antenna in each point after the whole stirrer rotation.

According to [2], an uncorrelated position occurs after the stirrer rotation large enough to cause the autocorrelation function to drop below the defined threshold, and is then uniformly distributed within the whole revolution. It is assumed that each subsequent position is uncorrelated, even though strong correlation might occur within the rotation, e.g. due to the symmetry of the stirrer [16]. The autocorrelation function calculated from the VIRC data, shown in Figure 2.10, drops quickly and remains on a very low level (before returning to 1 at the end of the dataset due to the periodicity introduced by the calculation method). The number of samples calculated using the ACF method for the RC is shown in Figure 2.11. The unpredictability of the flexible walls combined with the random motor movement creates a sequence so unordered that any periodicities are removed, and correlations are minimized, even if sample repetitions are still present in the data. For this reason, the application of the ACF method is not applicable, and other methods are investigated.

The Sample Difference method has been applied according to [26]. The results are shown in Figure 2.12. The results obtained in the RC are only a bit lower than using the ACF method (from Figure 2.11), proving that this method is in fact usable in this setup. The amount of presumably uncorrelated samples obtained in the VIRC is significantly higher, stretching from 1000 up to even 6000 in the upper frequency range. However, upon closer analysis of the rate of growth of the calculated number over time, shown in Figure 2.13, a linear trend is observed. Apparently, the number of samples grows unboundedly. The number of samples obtainable within a single stirrer rotation in the $\mathrm{RC}$ is reached in the first seconds using the VIRC. Although such a linear behavior is not impossible, it is physically unexpected, and results only from the imperfection of the discussed method. At least a small variation, e.g. in form of curving or saturation, is anticipated.

To apply the CLT method, the standard deviation of 40 mean values was calculated from combined eight spatial positions and five frequency stirring steps. The results are shown in Figure 2.14. A large underestimation of the 


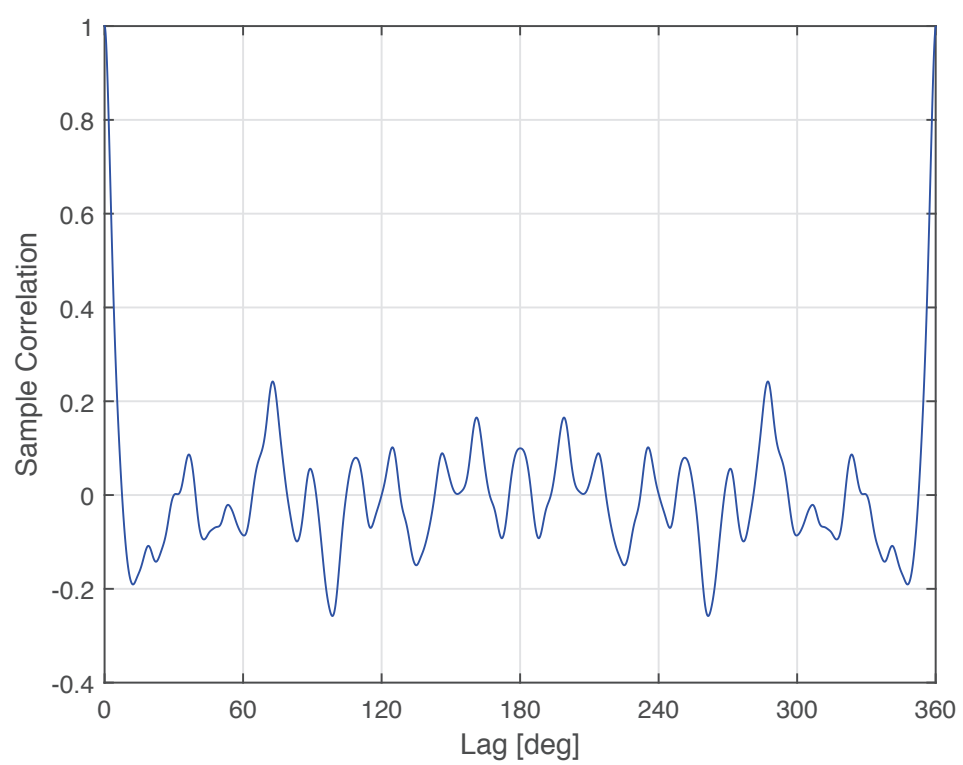

Figure 2.9: Example autocorrelation function behavior in the RC, $1 \mathrm{GHz}$.

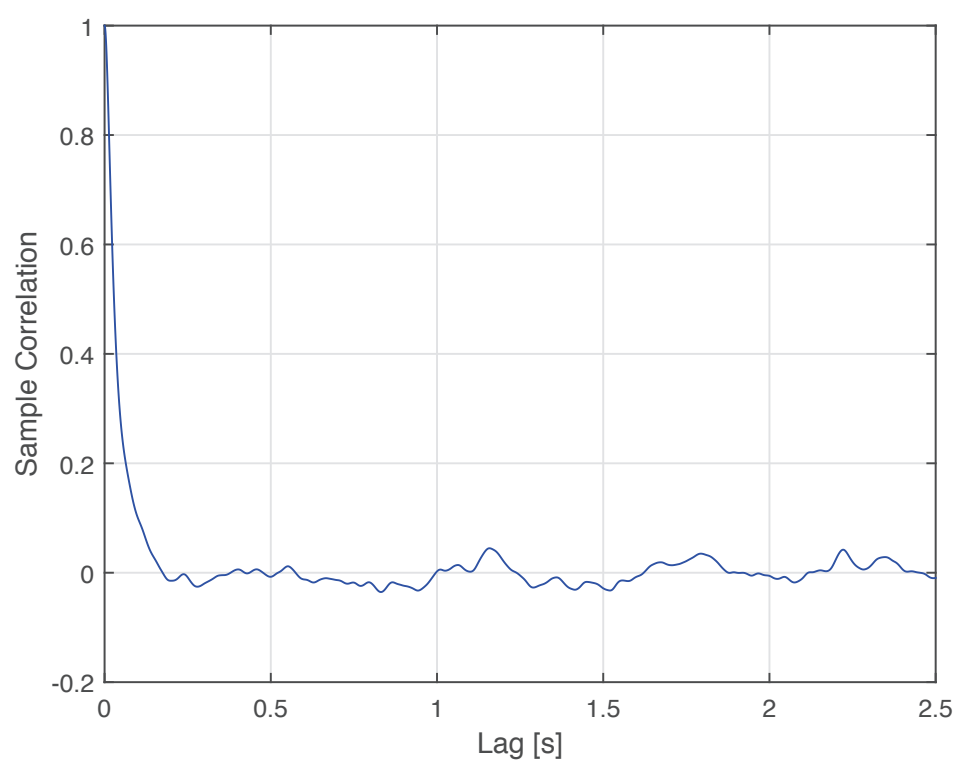

Figure 2.10: Example autocorrelation function behavior in the VIRC, 1 GHz. 


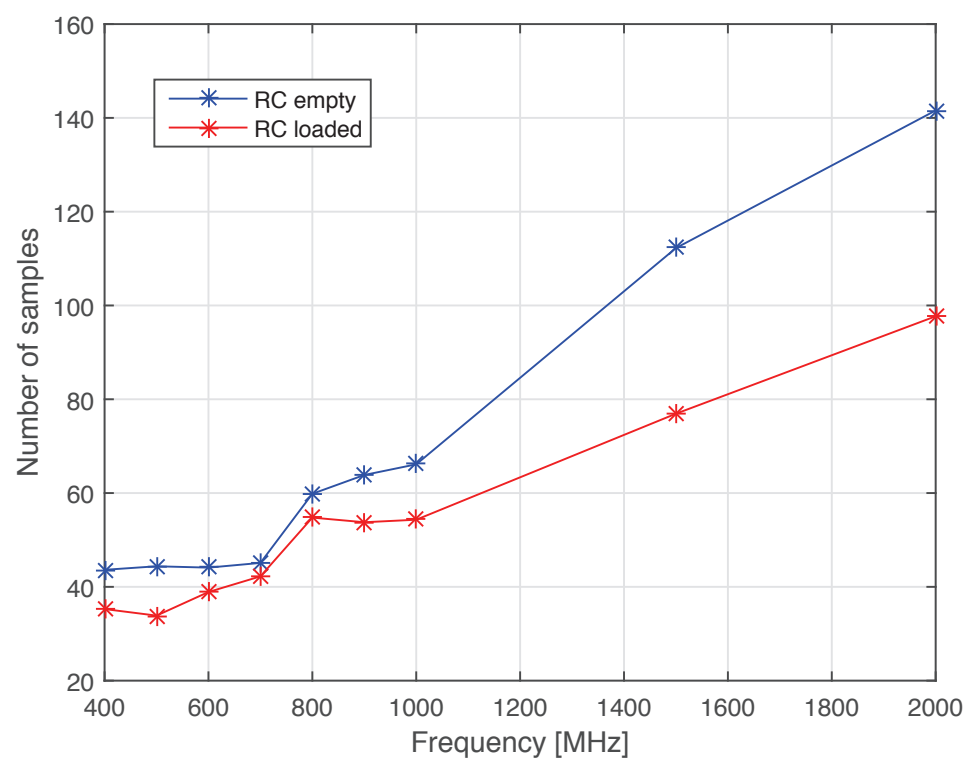

Figure 2.11: Number of uncorrelated samples using the ACF method.

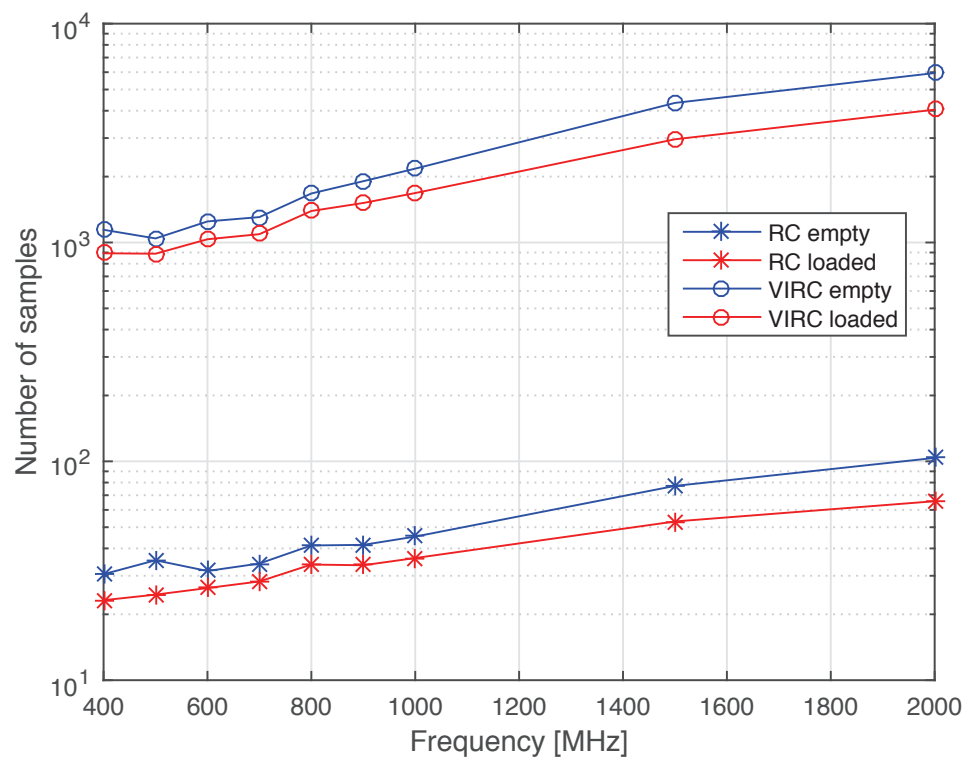

Figure 2.12: Number of uncorrelated samples calculated using the Sample Difference method. 


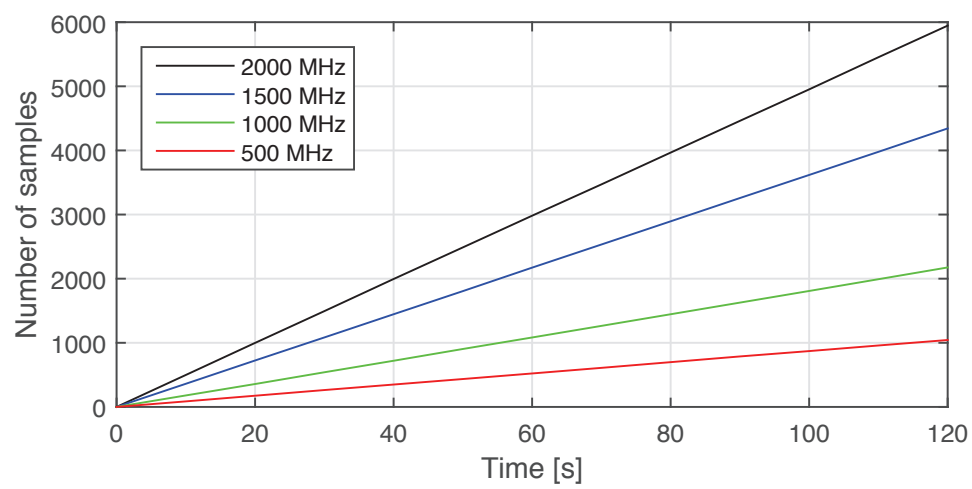

Figure 2.13: Number of uncorrelated samples calculated using the Sample Difference method as a function of time in the empty VIRC.

amount of samples with respect to the RC ACF method is observed, especially visible in the low frequency range, where the results are close to zero.

It is very important to point out that the CLT method is based on the estimation of the mean field strength throughout the chamber, which is very sensitive to the number of uncorrelated samples, as well as field uniformity, isotropy, and especially the LOS component. It is dependent not only on the theoretical accuracy of an estimation, but on the physical properties of the chamber as well. Additionally, a perfect case of the Rayleigh distribution is assumed for the calculation. Therefore, it is reasonable to consider those results as a qualitative way of evaluating the differences between the two analyzed chambers. Referring to the ACF results, which are around six times lower at $1 \mathrm{GHz}$ than the CLT ones, hypothetically multiplying the VIRC results by the same factor would imply that the latter chamber offers from 300 up to 1000 uncorrelated samples in the higher frequency range.

Closer analysis of the uncorrelated sample cumulation over time shows that the vast majority of the usable data is collected within the first 20 seconds of the measurement, and quickly saturates after reaching that point. However, it is shown in the later chapters that single but critical observations, e.g. very strong field values, can occur even an hour (and likely more) later, but being rare outliers, they do not significantly affect the estimation of the mean.

The $\chi^{2}$ test was selected for the RC and VIRC comparison because of its low sensitivity to data correlation, which is possible even in the sampled data. Although this test is presumed to provide reasonable results for differently sized datasets of correlated samples, like in the case of comparing $2.5 \mathrm{k} \mathrm{sam}$ ples from the RC to 12ok samples from the VIRC, initial tests have proven that such a great spread of sizes, as well as extreme oversampling leads to 


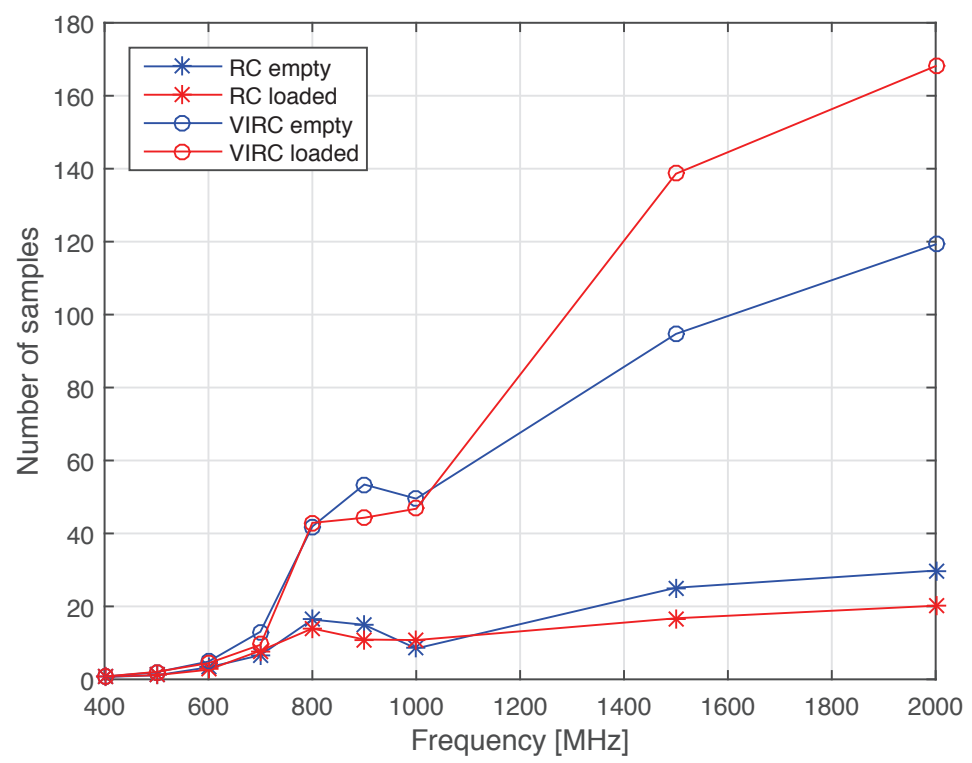

Figure 2.14: Number of uncorrelated samples calculated using the CLT method.

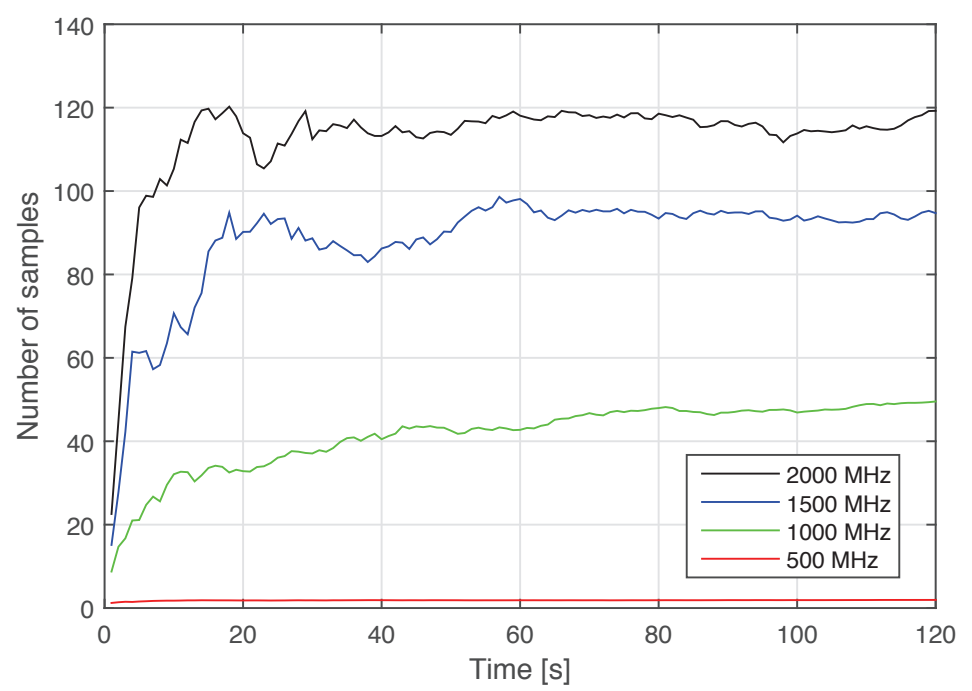

Figure 2.15: Number of uncorrelated samples calculated using the CLT method as a function of time in the empty VIRC. 
overestimated rejection. Therefore, 50 samples (around the expected amount of i.i.d. samples in the RC) were uniformly picked from both datasets for the analysis. Figure 2.16 shows the rejection rates calculated from the 40 datasets per frequency point. It can be seen that in the whole frequency range, the VIRC generally performs better. At low frequencies, the sample correlation might have a major impact on the test, however, it is not the case in the high frequency band, implying that the field mixing capabilities are a dominant factor. Repeating the test with similarly selected 100 samples further increased the difference in favor of the VIRC. On the other hand, the test performed on 25 sample sets significantly lowered the rejection rates in both cases.

\subsubsection{Reverberant environment analysis}

This subsection focuses on the analysis of two REs, a workshop and an office, as spaces potentially possessing significant reverberant properties. Under the hypothesis that these external environments can be comparable in behavior with the RCs available in laboratory conditions, similar tools have been applied as in the previous subsection. Although identical methods have not been used as before, the general behavior of the fields can be understood and compared by performing a simplified measurement set. Moreover, to create the missing link connecting the imperfect REs and the RCs optimized for laboratory use from the previous subsection, the simplified measurements have also been performed in a referential RC.

For the evaluation of the REs, the IL, the Q-factor, and the $\chi^{2}$ GOF test were selected as measures yielding a significant amount of information about the general behavior of the tested environments. These three commonly used measures can be calculated from the $S_{21}$ parameters without the necessity of having any other information about the environment. The ILs of the two REs along with the referential RC, calculated using equations 2.9 and 2.10, are shown in Figure 2.17. Two important observations can immediately be made. Firstly, the ILs of the referential RC are gradually lower than those of the workshop and the office. The minimum IL is lower than the average IL by around 8 to $10 \mathrm{~dB}$ in the whole frequency range. Secondly, the rate of change of the data in frequency is significantly higher in RC than in the other two sites due to a high Q-factor, although their variances are comparable. Also, the normalized variances of the average ILs are significantly lower than of the minimum ILs.

The Q-factors of the three analyzed test sites are presented in Figure 2.18. Although significantly lower than the empty RC, the composite Q-factors of the workshop and the office are comparable to those of a heavily loaded RC [31], indicating that reverberation can still be possible. This result also shows that the office or workshop are very far from free space behavior. 


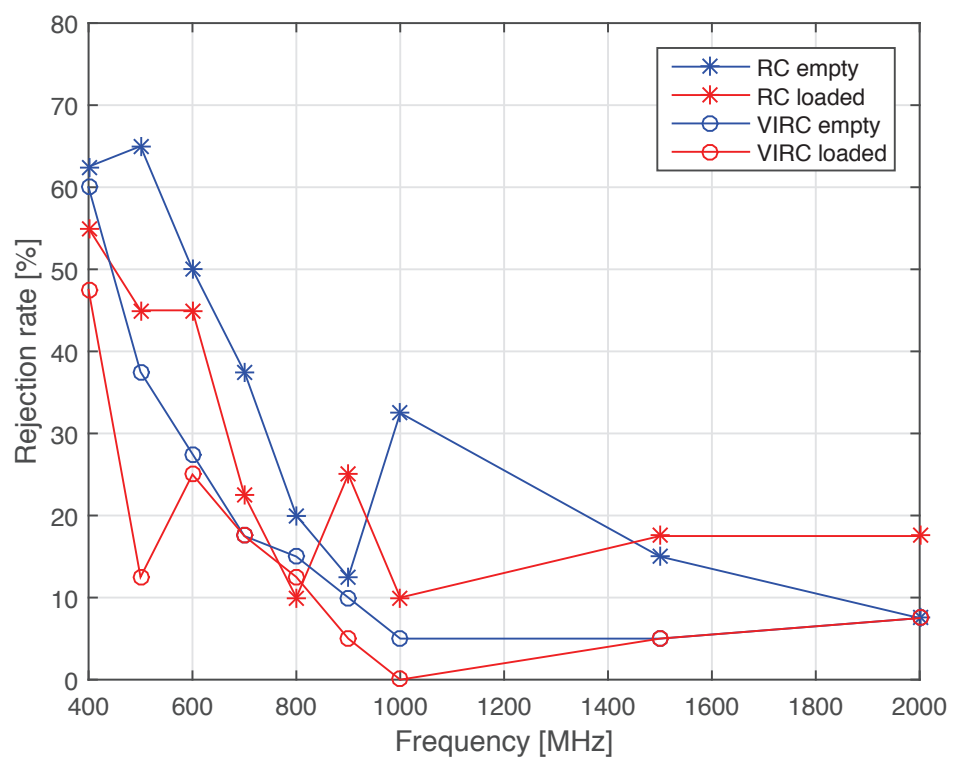

Figure 2.16: Results of the $\chi^{2}$ test performed on 50 samples selected from each dataset.

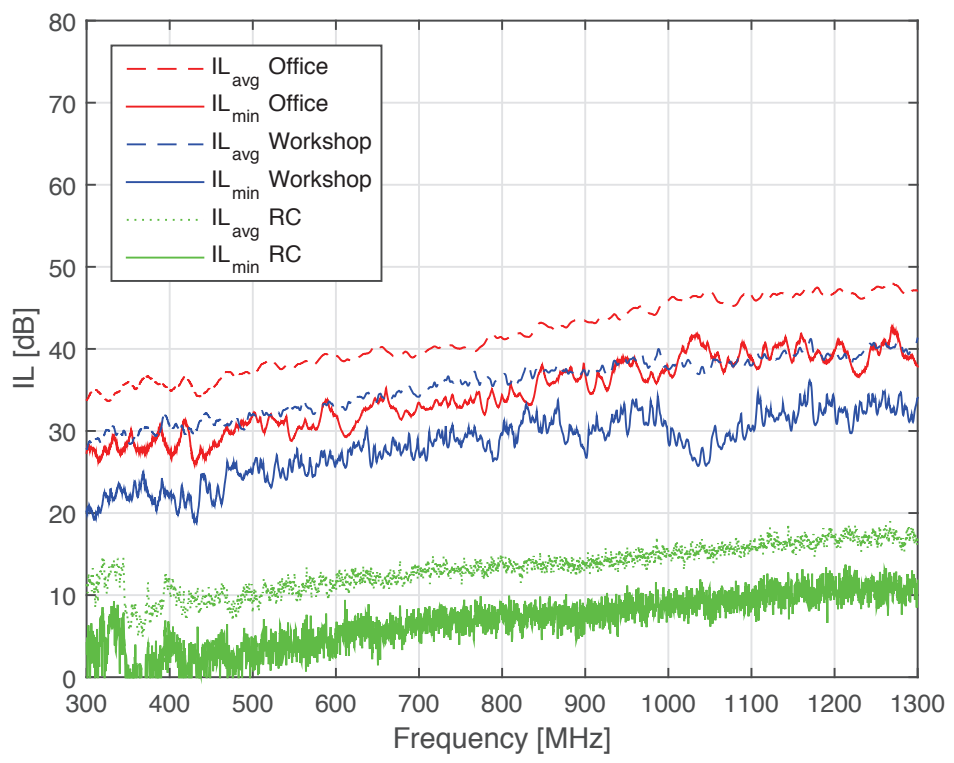

Figure 2.17: Minimum and average insertion losses for the three environments. 


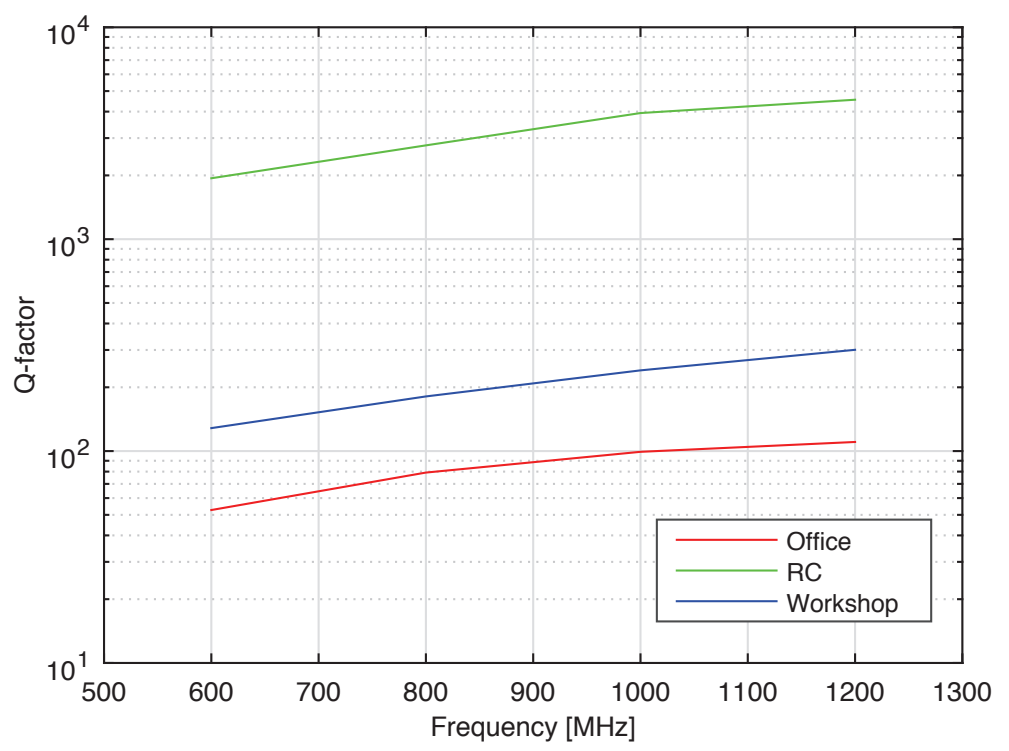

Figure 2.18: Composite Q-factors of the three environments.

Figure 2.19 shows the results of the $\chi^{2}$ test for the Rayleigh distribution, calculated from the 50 spatial positions. The rejection rates were calculated from the results of the tests performed for each frequency point within a $50 \mathrm{MHz}$ band. As expected, the test results from the RC are consistent over the whole frequency range, except when approaching the LUF. On the other hand, the workshop and office test results indicate serious absolute deviations from the theoretical Rayleigh model, especially apparent in the higher frequency range. Both environments exhibit significant periodic variations in frequency due to inconsistencies of the distributions. The latter observation is typical for semi-reverberant spaces, where single, high $\mathrm{Q}$-factor resonances or large apertures exist, which become dominant over the average diffused fields. Those cases can be compared to undermoded chambers [11], in which also only a limited number of significant modes is excited [32]. In such a case, the requirements regarding consistent statistical field uniformity and isotropy are less likely to be met. On the other hand, the test results in the REs often drop to values even lower than in the $\mathrm{RC}$, indicating a very good fit of the collected data to the theoretical model, thus express significantly high reverberation properties. Selected histograms representing each case are shown in Figure 2.20. 


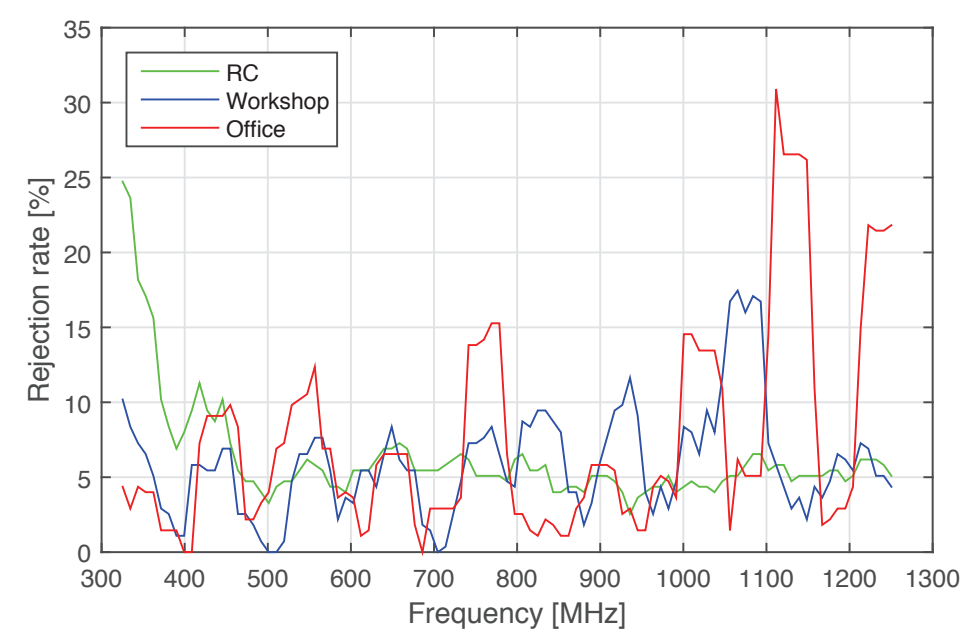

Figure 2.19: Rejection rates of the $\chi^{2}$ test within a $50 \mathrm{MHz}$ band.

\subsection{Summary and discussion}

The comparison of the classical RC and the VIRC has been performed in order to find the most suitable setup for modeling a reverberant environment, as well as better understanding of the behavior of EM fields therein. The similarity of the Q-factors indicates that the two chambers show high resemblance in terms of reverberation properties. The k-factor analysis performed in those chambers indicated a strong presence of the LOS component in the low frequency range, presumably due to the low directivity of the LPDA operating below its nominal band, rather than poor stirring efficiency. The differences of the k-factors in the two chambers are small, with a minor advantage of the VIRC. It has been proven that although the RC gives the possibility of reobtaining the samples via sample repeatability, its capabilities of generating new, uncorrelated samples by means of mechanical stirring are limited. On the other hand, the VIRC minimizes the possible correlations by maximizing the randomness through the unpredictable behavior of the flexible walls. Such a stirring mechanism allows to obtain very long sequences of unrepeated data, therefore possibly a very high amount of uncorrelated samples. Although the used Sample Difference and CLT methods do not give consistent results, they express a substantial advantage of the VIRC. This allows a much deeper analysis, as is described in the next chapter where the extreme values are sought. Finally, the performed $\chi^{2}$ test indicates that the distribution of the data collected in the VIRC is generally closer to the theoretical Rayleigh distribution, implying that the overall performance of this chamber is also better than the RC. The combined results indicate that the VIRC is a more 
suitable solution for further work, having better and more efficient stirring capabilities, but more importantly, providing a much higher amount of usable data, which plays a crucial role in the empirical analysis of the outliers, such as maximum field strengths in Chapter 3 .

The analysis of the reverberant environments, the office and the workshop, indicated that they in fact act more like poor reverberation chambers rather than free space environments as assumed e.g. in [33]. The existence of resonating fields has been proven by measuring the Q-factors, which are similar to the ones measured in a heavily loaded RC [31], allowing for a reasonable reverberation [34]. The average resonance strength is rather weak due to the high amount of losses present on site, e.g. windows, doors. Therefore, the transmitted power is highly attenuated, by 20 up to $50 \mathrm{~dB}$ depending whether average or maximum readings are considered. However, the distribution of the samples collected using volume sampling method can be compared to the theoretical Rayleigh distribution obtainable in a perfect RC. Due to the hypothetically nonuniform distribution of the resonance strengths, the $\chi^{2}$ test frequently fails in the office environment, but also passes, especially in the workshop. Therefore, it is concluded that even though the analyzed $\mathrm{RE}$ do not behave as a properly working $\mathrm{RC}$, they do posses the reverberant properties, allowing them to be analyzed, modeled, and utilized using the RC tools. 


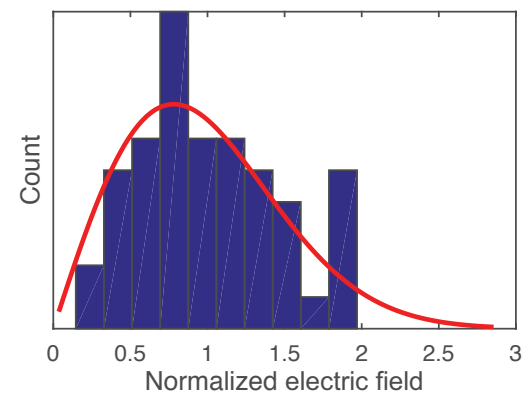

(a) $\mathrm{RC}_{705} \mathrm{MHz}$.

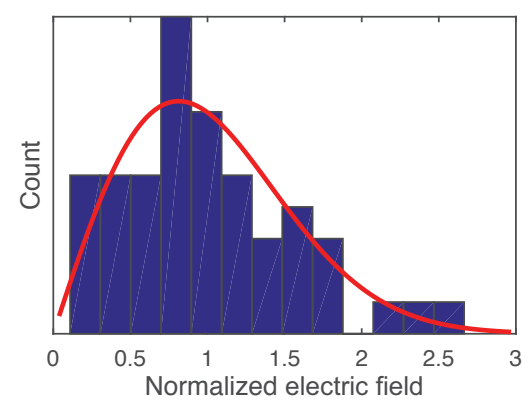

(c) Workshop $705 \mathrm{MHz}$.

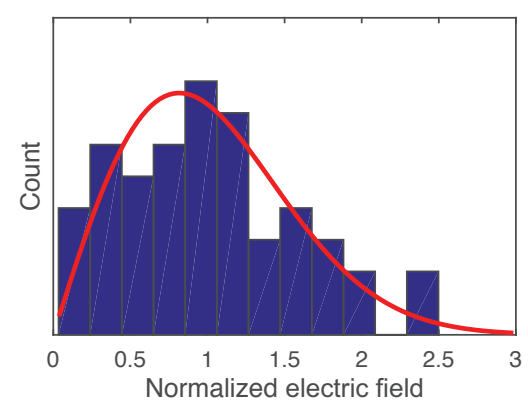

(e) Office $705 \mathrm{MHz}$.

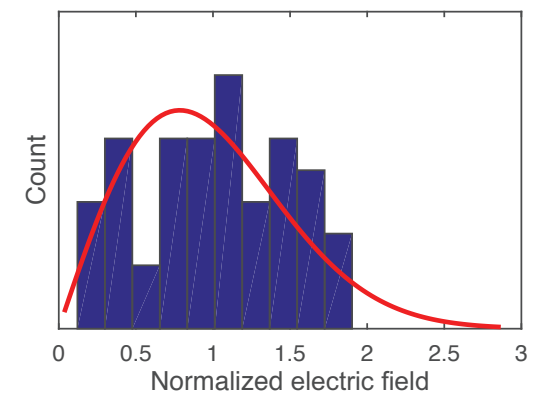

(b) RC $770 \mathrm{MHz}$.

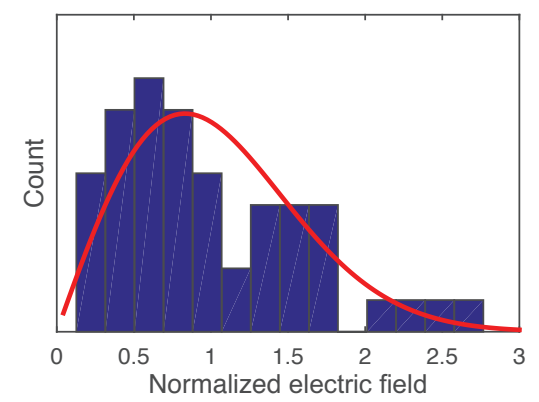

(d) Workshop $770 \mathrm{MHz}$.

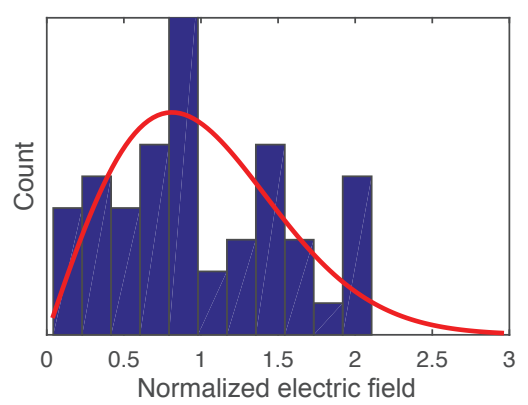

(f) Office $770 \mathrm{MHz}$.

Figure 2.20: Histograms with lower $(c, e)$ and higher $(d, f)$ rejection rates than the $R C(a, b)$. 


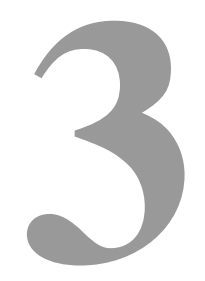

\section{Maximum field strengths}

\subsection{Introduction}

Any multipath environment modifies the injected energy in a way that is best described using statistical, rather than deterministic methods. The very high complexity of the critical parameters affecting the reflections enforces their approximations and utilization of simplified models. These models are usually accurate in the highly repeatable near-average region where decent estimations are possible. However, the outliers such as the maximums of the standing wave pattern are much more sensitive to the variations of the assumed parameters, as well as very difficult to observe due to their low probability. They play a great role in the EMI threat analysis because they not only occur as time transients in a changing environment such as an RC, but, by ergodicity, also as steady hot-spots, i.e. points in space of very high power density. Very strong field can be unpredictably created in any multipath, and especially resonating REs, including airplane fuselages, ships, or even buildings.

The fundamental model of field distribution, commonly recognized by the RC community, is based on the central limit theorem [9]. It states that the quadrature components of the field in a perfect RC are distributed Gaussian with zero mean, therefore the field magnitude in one dimension comes from the Rayleigh distribution. The same results are obtained from the plane wave 
integral representation (PWIR) [35], which states that the field strength in a given point is a summation of waves or modes coming from random directions, having random phases and random polarization. Under those assumptions, the summation of an infinite amount of modes has zero mean and a specified variance. Then, according to the maximum entropy method, it also results in a Gaussian distribution of the quadrature field components [18]. This model provides a reasonable reference, especially accurate in the near-average region, or in cases where strongly limited amounts of samples are obtainable, such as in commercial RC used for EMC testing.

On the other hand, the analysis of the field maximums is a difficult subject due to the very low probability of their occurrence, as well as high sensitivity to the theoretical assumptions. Many publications, e.g. [36], [37], [38], [39], [40], [41], [42], [43], are focused on deriving analytical expressions usable in finding the expected value of the maximum field strengths based on the aforementioned foundations. Although the PWIR is a very simple and powerful tool, improvements have been suggested [44], and deviations from the Rayleigh distributions have been reported to provide a better fit to the empirically obtained data [45]. In [29], the two-parameter Weibull distribution is suggested. It is defined as

$$
f\left(\left|E_{x}\right|\right)=\frac{b}{a}\left(\frac{E_{x}}{a}\right)^{b-1} e^{-\left(\frac{E_{x}}{a}\right)^{b}}
$$

where $\left|E_{x}\right|$ is the electric field strength in a single direction, $a>0$ is the scale parameter, and $b>0$ is the shape parameter. The distribution becomes Rayleigh (2.2) when $a=\sqrt{2} \sigma$ and $b=2$.

The Weibull distribution is even more apparent in case of undermoded chambers [46], although the borderline between the overmoded and undermoded chambers is rather blurred. Applying the Fisher-Tippett theorem [47], as the CLT equivalent for extreme values, led to defining the Generalized Extreme Value (GEV) distributions [48] [49] allowing to extend the parent distribution selection, also based on empirical data fitting.

A maximum is a single observation of the highest value from the given data set, e.g. stirrer rotation. Therefore, from the statistical point of view, it is directly bound to the amount of recorded samples. If this amount is limited to something physically obtainable in an $\mathrm{RC}$, the expected maximum is also a finite number. However, by definition, an infinite summation of finite random variables results in a distribution without the upper limit. Because it is physically impossible to create a field of infinite strength [50], the continuous distributions of the maximums are simply limited by an arbitrarily selected percentile. Being a common practice in statistics, this allows to create a tool that is usable in the vast majority of cases, providing 
highly reliable results. Additionally, apart from numerical simulations, it can be experimentally verified using the available tools, such as a classical RC, unfortunately delivering a strongly limited amount of samples. Although only the extremely improbable tails are removed from the distributions, such an operation does not find its rationale in physics. The equipment is sensitive to the maximum field strength, and once this field exceeds a certain threshold, EMI problems may occur. Given that the maximum field limit does exist, it is possible to find it and protect the equipment appropriately. Combined with high sensitivity to the imperfectly defined models that already include many assumptions, this topic became the main purpose of a deeper experimental analysis in this work.

The two most impactful factors differentiating a real $\mathrm{RC}$ and a theoretical model of a perfect chamber are the finite amount of summed components and the losses. Obviously, in the worst case of the PWIR when all of the incoming components add constructively, the field strength would be directly proportional to their amount. The maximum field is also directly affected by the Q-factor [38], but so is the average, therefore it is preferred to consider the maximum-to-average ratio (max/avg). If a loss mechanism is present, even an infinite summation of a resonating wave with itself losing a part of its energy with each bounce creates a geometrical series, which has an upper limit. If this is true for a single resonance, it is true for all of them. Even though [40] and [43] state that the max/avg does not depend on the environment properties, a counter hypothesis, has been created and aimed for experimental validation [51].

\subsection{Maximum field measurements}

The high amount of work done on the subject of maximum field addressed in the previous section is mainly focused on creating analytical models, utilizing the widely recognized theoretical fundamentals such as the CLT or PWIR. Although proper reasoning is given, the models generally inherit the same, sometimes brave assumptions. In the published results, they are validated only by means of numerical simulations (also having multiple, often the same assumptions) or highly limited experimental measurements. The latter solution, if done properly, is known to provide the information in its purest form, containing all recognized and unrecognized physical phenomena, without any unnecessary assumptions. Apart from any undesirable properties of the measurement equipment used, its greatest bottleneck lies in the capabilities of the measurement setup not being able to provide enough freedom in parameter variability and data collection, especially for statistical analysis. In RC techniques, this is very often the case because the classical chambers provide a 
limited amount of samples per mechanical stirrer rotation. Although demanding, this number can be multiplied by applying e.g. volume sampling, source stirring, or frequency stirring, e.g. as in [49]. In Chapter 2, it has been shown that the VIRC outperformes the classical RC, especially in the most desirable amount of generated samples. Although this number is not specified, it is greater than in the $\mathrm{RC}$, and unbounded by the stirrer rotation, possibly allowing for generation of new positions for a prolonged time under unchanged conditions. Therefore, performing long, 0.5-1 hour measurements became the general purpose of the experimental analysis presented in this section, exploiting the unique capabilities of the VIRC. The measurement campaign was split into three different parts: time dependency, Q-factor dependency, and antenna dependency, all focusing on isolating different parameters.

\subsubsection{Time dependency}

Initially, the behavior of the field over time is analyzed to verify the hypothetical usability of the VIRC for the chosen purpose, as well as provide a better understanding of the behavior of the fields in the creation of extreme values. Therefore, the setup was adjusted to collect highly oversampled data in order to decrease the probability of missing a potential maximum due to limited sampling frequency. At the same time, because the distribution of all data is analyzed, it is necessary to perform the measurement within a large dynamic range. For that, an AD8313 log-amp detector, similar to [52], was used. With very low response times [53] and $70 \mathrm{~dB}$ dynamic range this detector allows to observe anything that is expected to happen in the VIRC. The data was digitized by a 14 bit Data Acquisition (DAQ) system with $1 \mathrm{kHz}$ sampling frequency. A discone antenna was used for transmitting, and a double ridge guide (DRG) horn antenna for receiving, as shown in Figure 3.1. A single frequency of $1 \mathrm{GHz}$ used in this measurement was selected as a reference point, also allowing the $150 \mathrm{~cm} \times 120 \mathrm{~cm} \times 100 \mathrm{~cm}$ VIRC to perform well above the LUF.

Most of the publications on the subject referred to in the model overview section of this chapter relate the obtainable maximum value distributions directly to the amount of available samples. As shown in Chapter 2, the VIRC is potentially capable of creating an environment generating uncorrelated sequences of data indefinitely due to the unpredictable and complex movement of the flexible walls. Although the estimated amount of uncorrelated samples has been shown to be a limited number (using the most reasonable CLT method), it is important to note that, as mentioned before, the outlying values do not necessarily affect this estimation. In other words, extreme samples are considered outliers, and therefore can be created even once the estimation of the mean is saturated. 


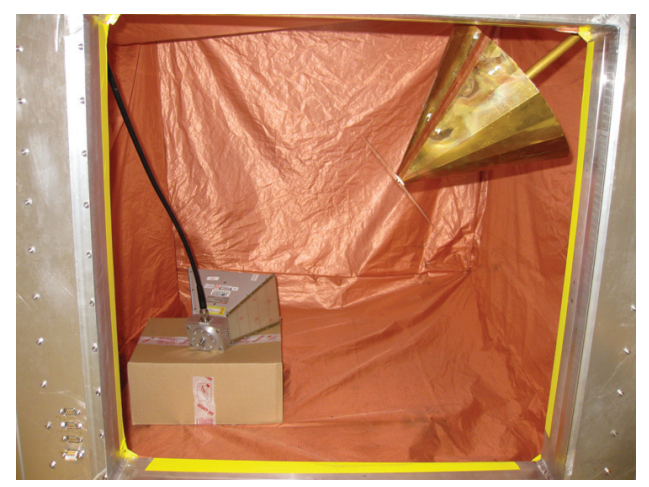

Figure 3.1: Layout of the antennas in the empty VIRC.

The recorded 1-hour electric field results, normalized to the average, are presented in Figure 3.2. It can immediately be observed that no apparent periodicities are present and the spikes representing local maximum values are non-uniformly distributed throughout the measurement. Even more importantly, the occurrence of the strongest spikes is very rare. In Figure 3.3, the behavior of the cumulative normalized maximum value is presented. It can be seen that even if the estimation of the uncorrelated samples using the CLT method saturates after around 100 seconds as shown in Chapter 2, Figure 2.15, the total maximum value is still increasing. The increment is the fastest within the first 200 seconds but does not stop after that point. The strongest field observed during this measurement appeared after 52 minutes, giving a max/avg ratio of 3.97 or around $12 \mathrm{~dB}$, shown in Figure 3.4.

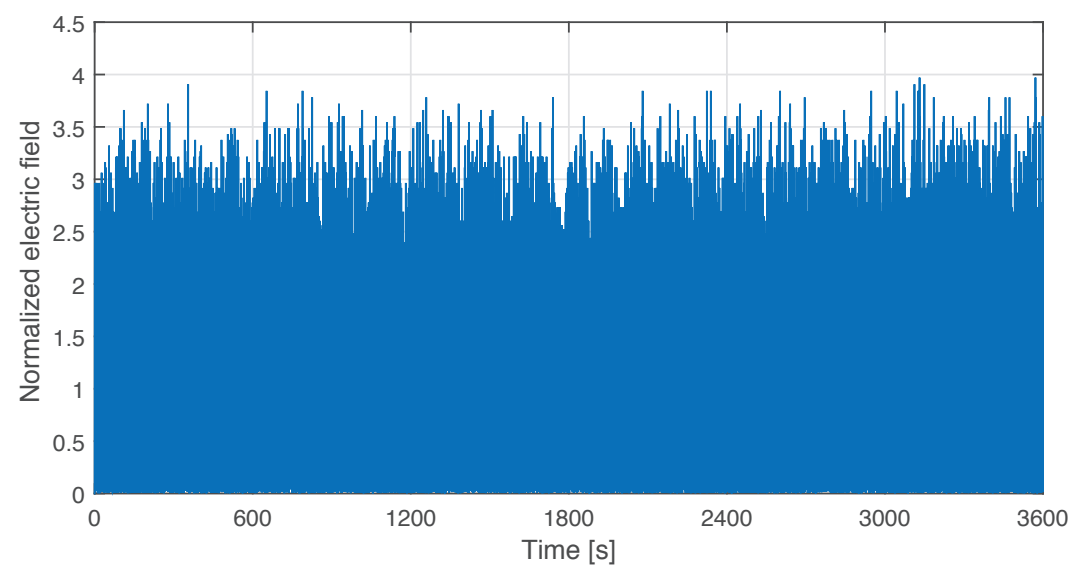

Figure 3.2: Whole 1-hour measurement normalized to the average. 


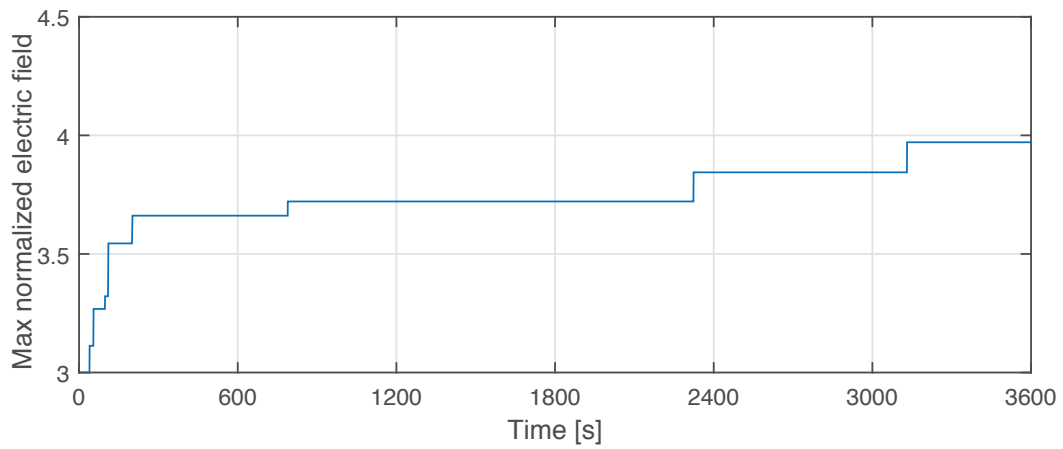

Figure 3.3: Cumulative maximum normalized to the average.

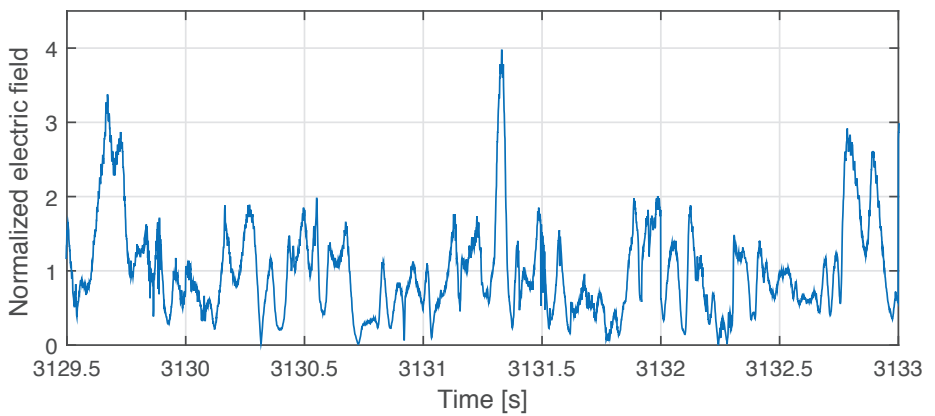

Figure 3.4: Occurrence of the highest value measured.

Although the maximum is expected to increase further with time, the rate of change becomes very low, and even after 30 minutes, a very high repeatability of the maximums is obtainable. Similar results with deeper analysis of another dataset are presented in [54]. It is concluded that this method of measuring the max/avg ratio under different conditions is appropriate.

\subsubsection{Q-factor dependency}

Under the hypothesis regarding the dependency of the max/avg on the losses of the environment, the VIRC setup was modified to allow freedom of varying the Q-factor. Initially, a front hatch with a variable aperture size, as shown in Figure $3.5 \mathrm{a}$, was selected as a solution giving the ability to control the chamber losses in a large range, and with a high resolution (e.g. every $5 \mathrm{~mm}$ ). However, opening the Faraday cage caused the outside noise to couple inside the chamber causing errors in the sensitive measurements. Placing the VIRC 
inside a fully anechoic chamber would be a reasonable solution but it was not available at the time. Although more effortful, giving less control and lower resolution, but commonly done in RC measurements, a method of loading the VIRC with pyramidal foam absorbers, shown in Figure 3.5b, was selected as a method to reliably alter the Q-factor. Pyramidal carbon-loaded foam absorbers are highly effective even if cut into pieces, therefore even only a tiny amount is capable of changing the Q-factor significantly. This solution, along with the variable hatch aperture, represent concentrated losses which are commonly met in typical REs in form of doors, windows. Hypothetically, they affect single modes, leading to an increased spread of the Q-factor distribution, and also affecting the field isotropy. Although better representing the imperfect REs, they are expected to lower the measurement repeatability due to additional variability of the results on the loss positioning. A solution to create distributed losses, i.e. losses affecting all the modes uniformly, was introduced and is presented in Figure 3.5c. Unfortunately, the absorbing foil limited the freedom of movement of the chamber walls, significantly crippling its operation and rendering this method unusable. Therefore, loading the VIRC with variable pyramidal absorbers became the preferred measurement configuration, published in [55].

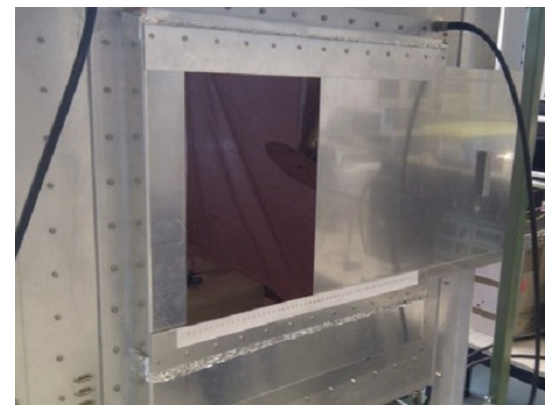

(a) Variable hatch aperture.

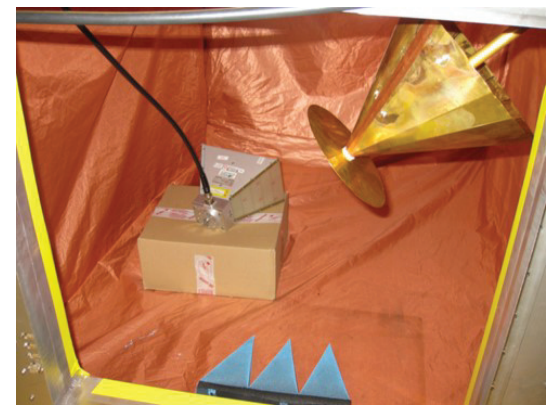

(b) Pyramidal foam absorber loading.

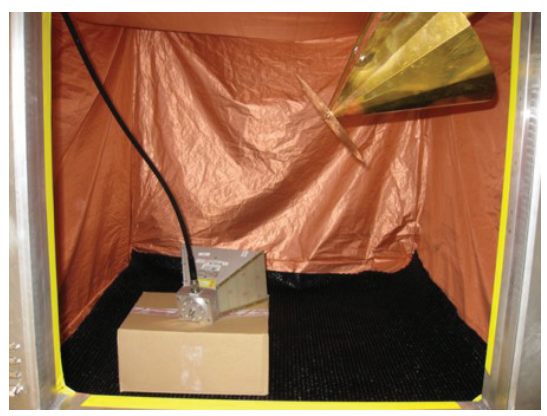

(c) Absorbing plastic foil loading.

Figure 3.5: Solutions to vary the Q-factor of the VIRC. 
Apart from altering the chamber losses, according to Equation 2.5, the Qfactor is also a function of two other parameters: volume (related to physical size) and frequency (related to electrical size). Therefore, the measurements were also conducted in a larger version of the VIRC with dimensions: $5 \mathrm{~m} \times 3 \mathrm{~m} \times 3 \mathrm{~m}$, moved by a single continuously rotating DC motor, creating configuration nr. 2. The materials of which both chambers are made are the same, so assuming that the wall losses are the dominant loss factor, the Q-factor of the empty big VIRC, according to [56], was expected to be as high as 17129. However, due to very poor shielding effectiveness of the improvised entrance to the chamber, certain wall connections, and lack of proper cable interfaces, the measured Q-factor in the empty chamber was 1746. To extend the measurement series even further, in the third configuration the same big VIRC was used at the frequency of $2.5 \mathrm{GHz}$, resulting in the increase of the Q-factor up to 3839. The results from configurations number 2 and 3 have been published in [31]. It is crucial to mention that, according to [57], with every introduced change, the mode density was greatly increasing, which is also related to the amount of summed components in the PWIR model. The empty chambers in the three configurations are compared in Table 3.1. Moreover, because in the VIRC the walls are actually stirring the field, with changing both the size of the chamber and frequency, the number of independent samples was expected to increase [58][25].

Table 3.1: Measurement configurations.

\begin{tabular}{|c|c|c|c|}
\hline & Config. 1 & Config. 2 & Config. 3 \\
\hline Setup & Small VIRC & Big VIRC & Big VIRC \\
\hline Frequency & $1 \mathrm{GHz}$ & $1 \mathrm{GHz}$ & $2.5 \mathrm{GHz}$ \\
\hline Dimensions [m $\times \mathbf{~ m ~ x ~ m ] ~}$ & $1.5 \times 1.2 \times 1$ & $5 \times 3 \times 3$ & $5 \times 3 \times 3$ \\
\hline Measured Q-factor (empty) & 2026 & 1746 & 3839 \\
\hline Mode density [per MHz] & 1.7 & 69.8 & 436.3 \\
\hline
\end{tabular}

In configuration 1 , the amount of inserted losses varied from nothing to one whole block of a cut absorber in 1o steps, as shown in Figure 3.6. In configurations 2 and 3, the chamber was loaded with o to 12 boxes with absorbers in 7 steps each, as in Figure 3.7. This allowed to change the Q-factor from around 125 (in config. 1) up to almost 4000 (in config. 3). The loads were distributed all around the chamber in a random manner, but avoiding the main lobes of both antennas. The equipment and measurement techniques used were the same as in the previous section, totaling in 24 hours' worth of measurements shown in this work. 


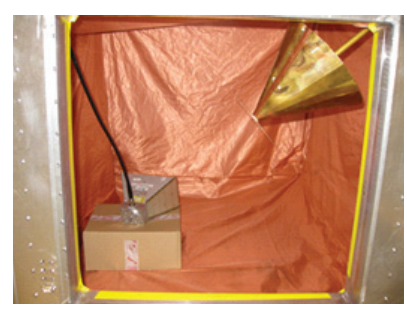

(a)

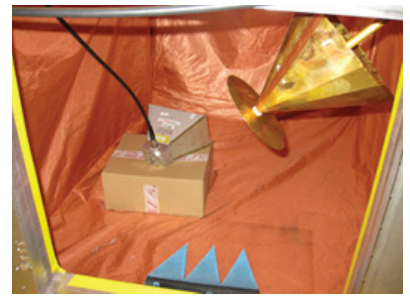

(c)

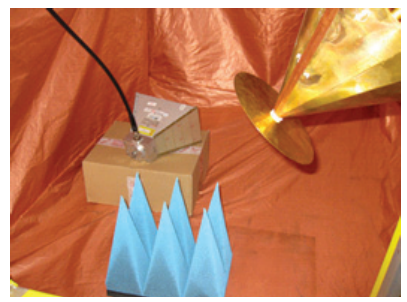

(e)

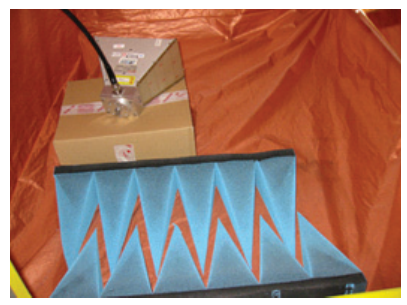

(g)

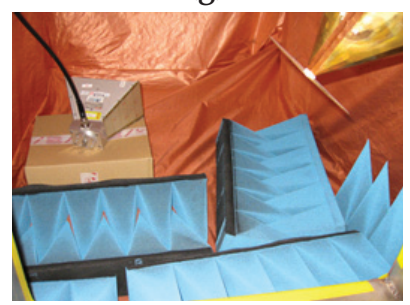

(i)

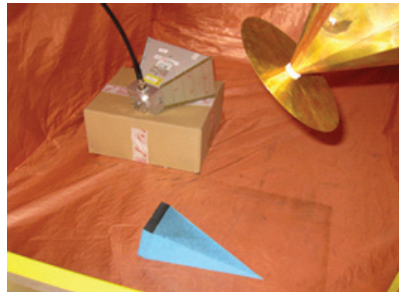

(b)

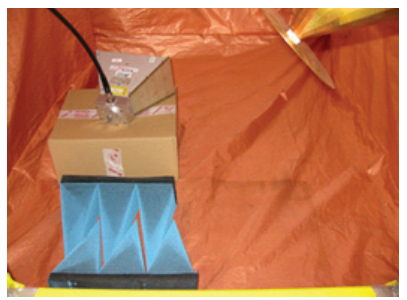

(d)

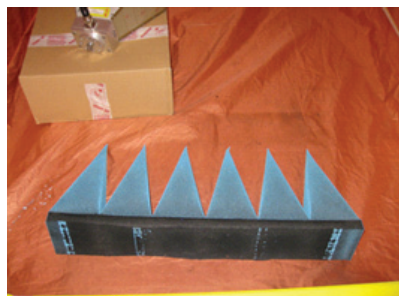

(f)

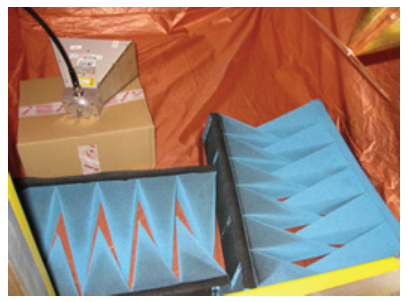

(h)

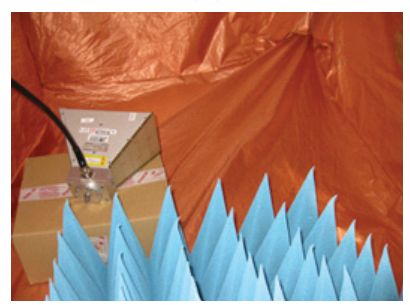

(j)

Figure 3.6: Layout of absorbers inside the VIRC used for config. 1. 


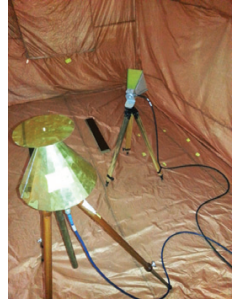

(a)

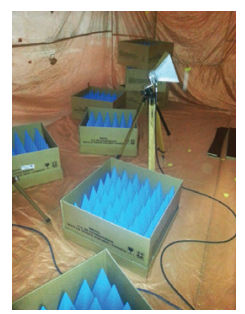

(d)

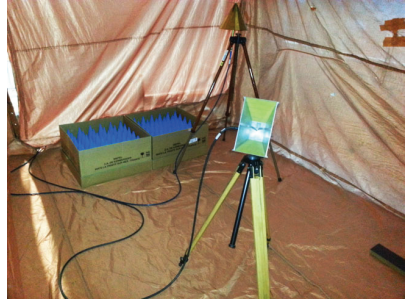

(b)

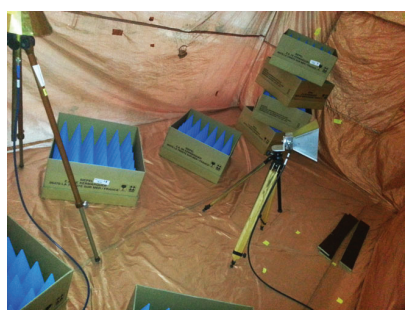

(e)

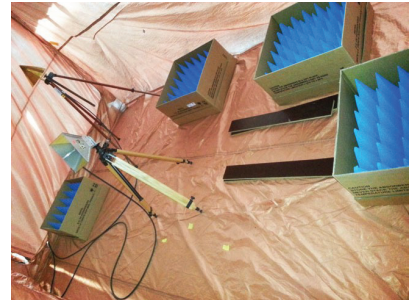

(c)

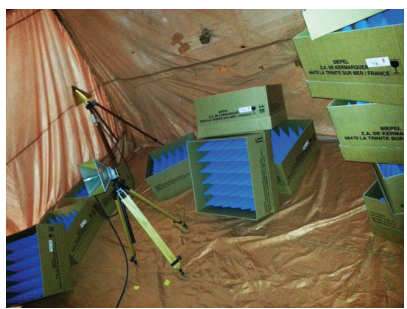

(f)

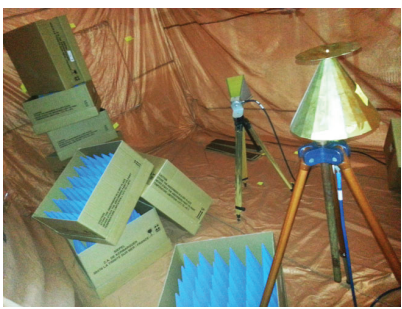

(g)

Figure 3.7: Layout of absorbers inside the big VIRC used for config. 2 and 3.

The very high amounts of absorbers can significantly affect the performances of the chambers. Therefore, to verify the proper operation in every case, $\chi^{2}$ GOF tests were performed on all of the recorded data to check a fit to the theoretical Rayleigh curve. The rejection rates, shown in Figure 3.8, were calculated from 500 picks of 50 random samples from the whole 3.6 Msample datasets. Apparently, and in accordance with [34], the Q-factor changes in the given range do not impair the reverberant properties of the VIRCs, giving constantly low rejection rates around 5\% (considered here as a systematic error of the chosen method), very comparable to the RC results from Figure 2.19 (with Q-factor of around 4000 at $1 \mathrm{Ghz}$ ) in Chapter 2. Curiously, an obvious outlier is present, showing an almost $15 \%$ rejection rate for the highest Q-factor in configuration 1 , which is the empty small VIRC.

The culprit histogram is shown in Figure 3.9b. A closer analysis indicates a negative skew of the data with respect to the theoretical Rayleigh curve, which increases the likelihood of failing the test. These results are compared with 


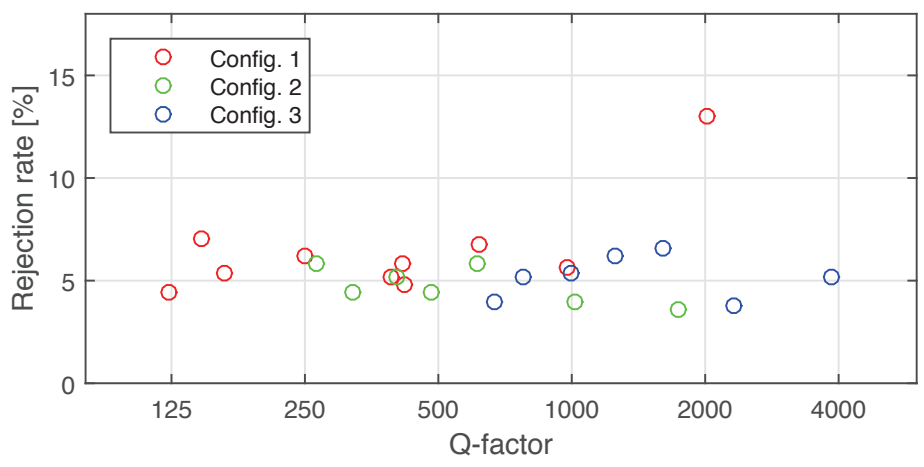

Figure 3.8: Rejection rates of the $\chi^{2}$ GOF test performed on 500 random picks of 50 samples from the 24 1-hour datasets.

similar ones obtained in Chapter 2, using an USRP receiver, shown in Figure 3.9a. A similar skew is present, although it is seemingly less obvious, just like the tail of the distribution. A difficulty of comparing the shown VIRC results with their RC equivalents arises due to the highly limited amount of samples obtained in the latter, thus making the observation less apparent. Loading the chamber even in the smallest degree (here, with a single pyramid of an absorber) causes the skews to vanish, and a very good fit to the assumed theoretical Rayleigh curves is obtained, as seen in corresponding Figures 3.9c and 3.9d. This phenomenon has been targeted and tested e.g. by validating the setup parameters such as detector linearity (by varying the input power), but no differences were observed. A similar issue has been addressed in [59], also referring to [6o], as well as in [61]. Changing the antennas indeed had an effect and is discussed in the next section. On the other hand, in [62] it is implied that such behavior could indicate that the chamber is undermoded. Although possible because the VIRC is rather small (which affects mode density), while having a rather high Q-factor (which affects mode bandwidth), and the mode overlap could indeed be the limiting factor, it is not expected at the frequency of $1 \mathrm{GHz}$, and with such a high repeatability. ew

The results of the calculated max/avg ratios for all 24 datasets are presented in Figure 3.10. A 12-13 dB ratio is obtained uniformly throughout the Q-factor values ranging from 250 up to 4000 , overlapping in all three configurations. The values shown are up to $1 \mathrm{~dB}$ higher than in [31] due to increased sampling rate of the processed data ( $1 \mathrm{kHz}$ here vs. $100 \mathrm{~Hz}$ in the reference). The three outlying results for the lowest end of configuration 1 indicate a $11 \mathrm{~dB}$ max/avg ratio. From the closer analysis of the setup under these conditions it has been concluded that the very high amounts of material placed inside the small VIRC impaired its field stirring abilities by limiting the range and 


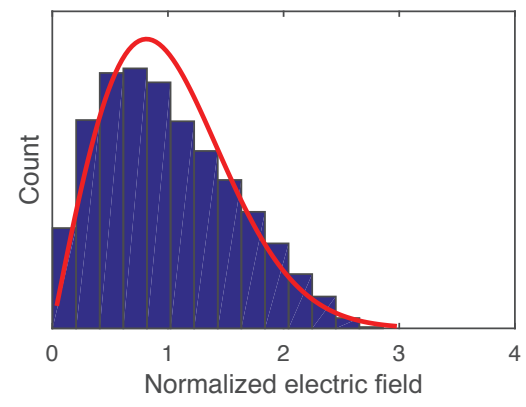

(a) Empty VIRC, Ch.2.

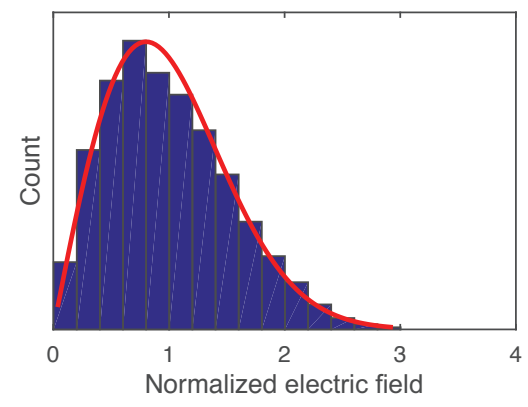

(c) Loaded VIRC, Ch.2.

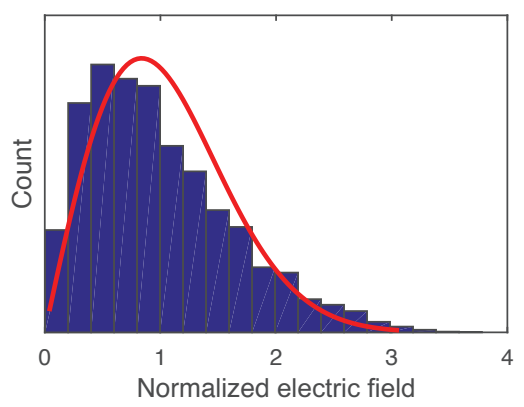

(b) Empty VIRC, Ch.3.

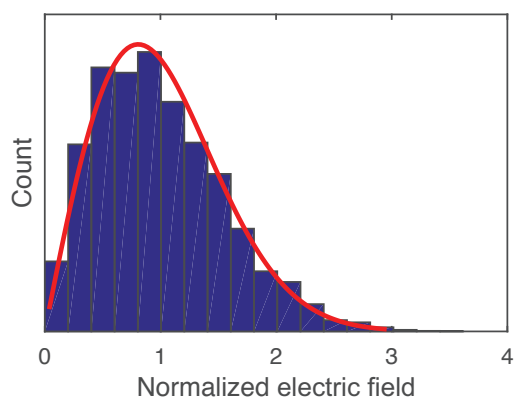

(d) Loaded VIRC, Ch.3.

Figure 3.9: Histograms obtained using the setups from Chapter 2 (USRP, Tx: LPDA, Rx: discone) and Chapter 3 (AD8313, Tx: discone, Rx: DRG).

freedom of movement of the flexible walls, thus providing a smaller amount of new positions. Although the obtained histograms in those cases successfully pass the GOF test, their tails are significantly shorter. Taking the $99^{\text {th }}$ or $95^{\text {th }}$ percentile removes the outliers, therefore lowering the final result, but allows to observe the change in behavior of the data more reliably. The max/avg results are almost flat throughout the whole analyzed range with a single exception. After performing 24 1-hour measurements using the VIRC - a setup dedicated for measuring fast changing fields, optimized for the highest available uncorrelated data generation, it can be stated that the hypothesis tested in this section is rejected. The Q-factor does not influence the max/avg ratio. The three configurations creating environments with very high spread of Q-factors, mode densities, numbers of samples, and general loss mechanisms, yielded the same results, highly comparable with [43], providing a proof for the correctness of [43], saying that the max/avg ratio does not depend on the composite Q-factor of the chamber. However, the aforementioned histogram skew led to a comprehensive investigation including tests with different antennas. From the initially collected data, a new hypothesis was created, 
saying that the max/avg ratio depends on the properties of the receiver, in form of antennas or an EUT. The new hypothesis is addressed in the following section.

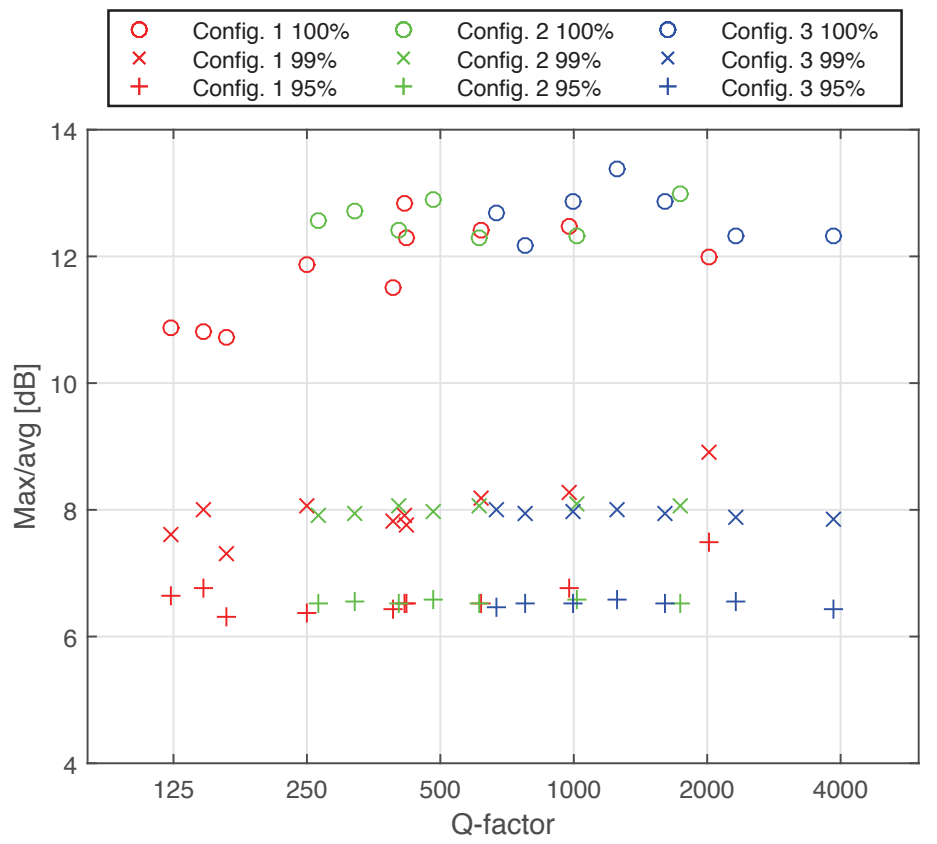

Figure 3.10: Max/avg results for the three configurations described in Table 3.1, for different percentiles.

\subsubsection{Antenna dependency}

Due to the distribution skew of the data measured in the previous section, a necessity arised to carry out an investigation to better understand the reason for the outlying results. The work presented in [6o] and more recent [59] address the issue of obtaining a different distribution than Rayleigh, depending on the selection of antennas. Apart from that, a relation between the antenna aperture and statistics of the recorded field is expected on the basis of a deviation from the theoretical assumptions of a infinitesimal (point) antenna as assumed in the PWIR theory. 


\section{Initial tests}

Initially, the measurements were performed using the same technique and setup as in the previous section, i.e. one hour data recording using the AD8313 detector at $1 \mathrm{GHz}$ frequency, but in the empty chamber and with different antennas. For transmission, the highly directional DRG horn antenna was used instead of the discone to minimize the k-factor, which although very small, could critically affect the distribution shape. For receiving, a monopole antenna with a $50 \mathrm{~mm}$ diameter ground plane, shown in Figure 3.11, was built, allowing to easily change the length of the active element. The selected lengths of the monopoles were: $10 \mathrm{~mm}, 25 \mathrm{~mm}, 50 \mathrm{~mm}, 75 \mathrm{~mm}$ (resonant at $1 \mathrm{GHz}$ ), and $100 \mathrm{~mm}$. Although the Q-factor is expected to decrease in case of matched antennas, this mechanism was not dominant and had no noticeable effect, resulting in very similar Q-factor results throughout the five measurements using different antenna lengths.

The results of the $\chi^{2}$ GOF test performed on 500 random picks of 50 samples from the five 1-hour measurements using different antenna lengths are shown in Figure 3.12. The results cover the range where the observed outlier from Figure 3.8 was present, reaching up to $30 \%$ rejection in case of the shortest, $10 \mathrm{~mm}$ monopole. The best fit of the data to the Rayleigh curve, below $10 \%$ rejection, is obtained for the matched antenna, i.e. $75 \mathrm{~mm}$ monopole. The histograms representing the two cases are shown in Figure 3.13, indicating a reproduction of the previously observed skew. Additionally, it can be seen that the tail of the short monopole histogram is significantly longer than in the resonant case. The calculated max/avg ratios for the 5 analyzed lengths, shown in Figure 3.14, follow a similar trend as the $\chi^{2}$ rejection rate, binding higher max/avg values to the increased deviation from the Rayleigh curve. The lowest max/avg ratio, around $10 \mathrm{~dB}$, is obtained for the matched case. Shortening the monopole leads to a significant increase in the max/avg even by $6 \mathrm{~dB}$ in case of a $10 \mathrm{~mm}$ antenna. Lengthening the monopole has a milder but still visible effect. The shown $99^{\text {th }}$ and $95^{\text {th }}$ percentiles indicate that the distribution is in fact changed in a repeatable manner, especially for the shorter antennas.

\section{Extended tests}

Due to the unexpectedly high variation of the max/avg ratios, the results obtained needed a verification as well as an extension of parameter spread. The previously used monopoles had a rather small ground plane, possibly allowing the connecting cable shield to act a part of the antenna [63]. In this order, an entirely new measurement setup was prepared utilizing different technique and equipment thus eliminating any potentially influential imperfections of 


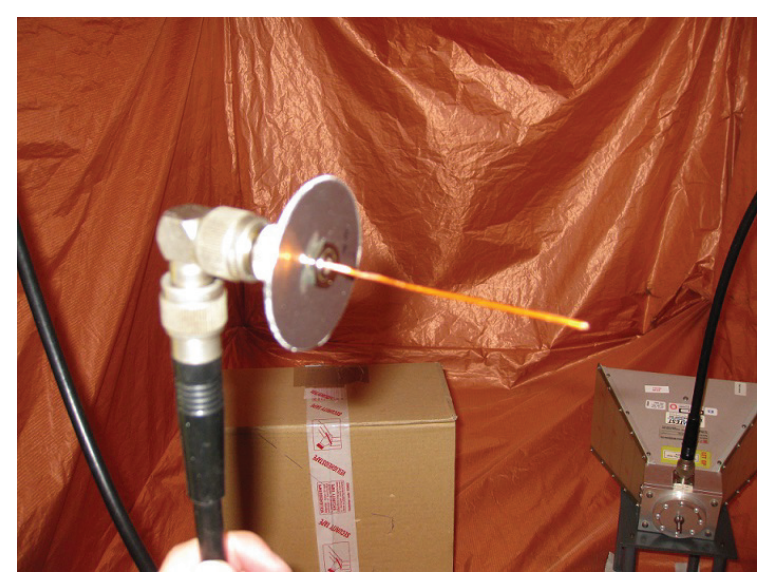

Figure 3.11: Monopole antenna with a $50 \mathrm{~mm}$ diameter ground plane used in the AD8313 setup.

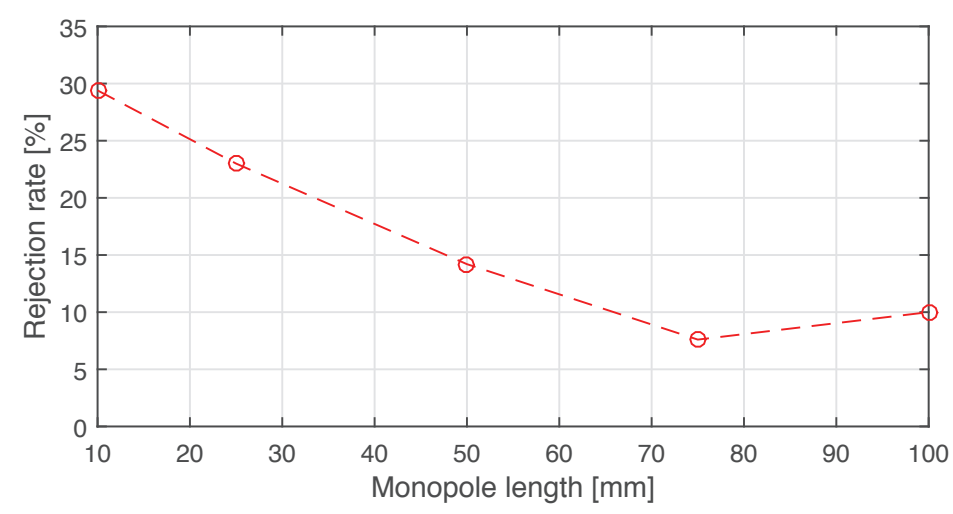

Figure 3.12: Rejection rates of the $\chi^{2}$ GOF test performed on 500 random picks of 50 samples from the five 1 -hour datasets measured using different monopoles (AD8313 setup).

the previous setup. The measurements were still performed in the same VIRC due to its preferred, aforementioned reverberation properties over the classical RC. Based on the results from the previous section, the setup was modified to include the $2.5 \mathrm{GHz}$ frequency point and take measurements in a slightly loaded chamber to minimize the (already unnoticeable) influence of the variable Q-factor due to antenna matching. Instead of the AD8313 detector, a spectrum analyzer (SA) with a tracking generator was used. It was performing $200 \mathrm{~ms}$ sweeps of 401 points in a $60 \mathrm{MHz}$ band around the central frequency, every $300 \mathrm{~ms}$, using sample detector. The SA does not allow 


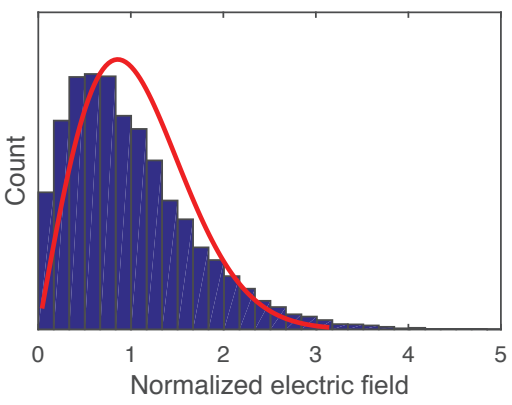

(a) $10 \mathrm{~mm}$ monopole.

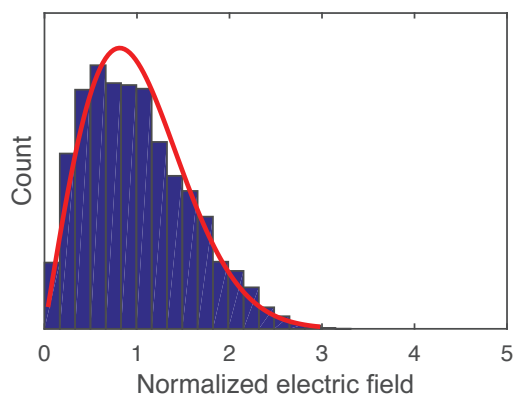

(b) $75 \mathrm{~mm}$ monopole.

Figure 3.13: Histograms obtained using a short (a) and a resonant (b) monopole (AD8313 setup).

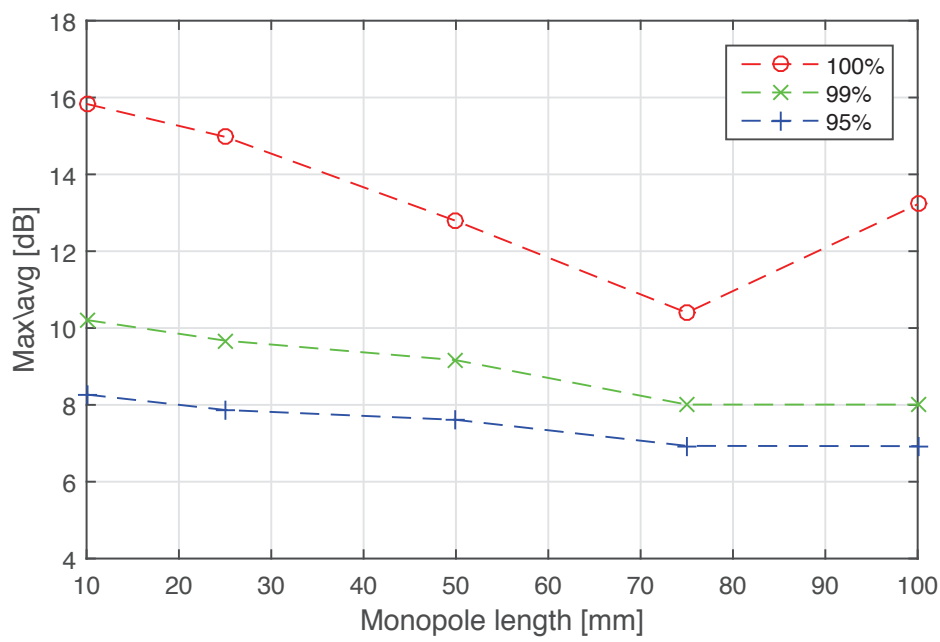

Figure 3.14: Max/avg results for different monopole lengths (AD8313 setup), for different percentiles.

oversampling in the time domain, i.e. recording the envelope of the signal as in Figure 3.4 because the effective sample rate in time is $0.33 \mathrm{~Hz}$. However, because in this investigation, the order of the samples is not as important as the their statistics, such a solution is even preferred as it provides a significantly more data than a single frequency time domain measurement. Therefore, 30-minute measurements were performed. All results were normalized to the average obtained from the 6000 sweeps, individually for each of the 401 frequency points to avoid any variances in the tracking generator and antenna frequency characteristics. Furthermore, to ensure that no nonlinear effects are 
present, the measurements were verified with different output powers but no differences were observed. The antenna lengths were selected to cover a wide range in logarithmically uniform steps, around and including resonant lengths for $1 \mathrm{GHz}$ and $2.5 \mathrm{GHz}$, as shown in Figure 3.15. The monopoles were mounted on a large $350 \mathrm{~mm} \times 350 \mathrm{~mm}$ square ground plane visible in Figure 3.16.

The $\chi^{2}$ GOF tests were performed on 50 samples, randomly selected five times from each of the 401 frequency points in the $60 \mathrm{MHz}$ band, i.e. 2005 times. Figure 3.17 shows that the rejection rates calculated for the $1 \mathrm{GHz}$ case are much greater than the equivalent ones from Figure 3.12 outside of the resonance. The results for $75 \mathrm{~mm}$ are highly comparable with the initial measurements, being rejected below 10\% of the time. As already observed in the previous section, in Figure 3.8, loading the chamber lowers the overall rejection, while the increased frequency results in uniformly low results for all monopole lengths, in both loaded and unloaded cases.

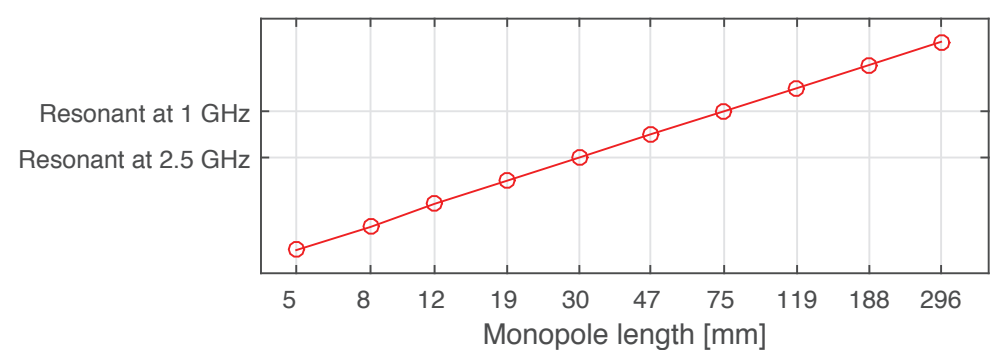

Figure 3.15: Monopole antenna lengths selected for the SA setup.

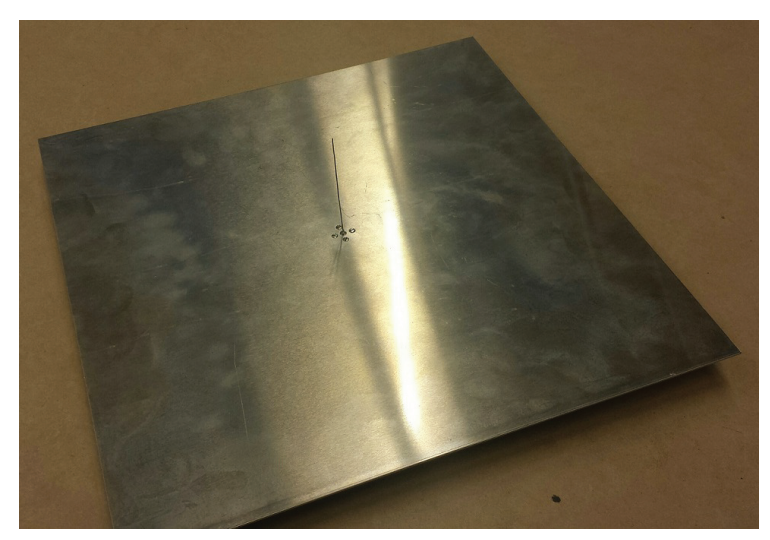

Figure 3.16: Monopole antenna with a $350 \mathrm{~mm}$ x $350 \mathrm{~mm}$ ground plane used in the SA setup. 


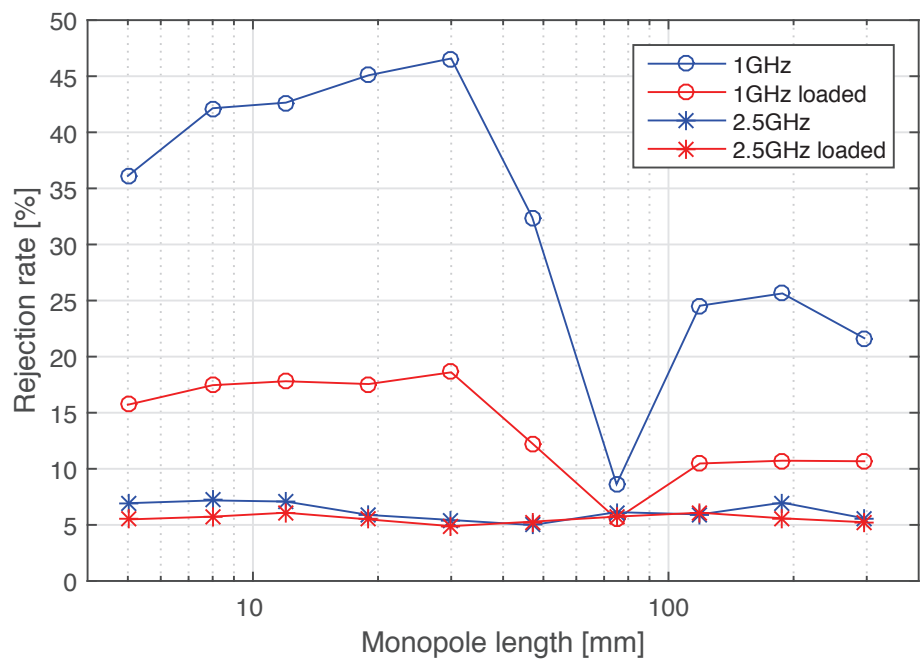

Figure 3.17: Rejection rates of the $\chi^{2}$ GOF test performed on five random picks of 50 samples at 401 frequency points in a $60 \mathrm{MHz}$ band for different monopoles (SA setup).

The max/avg results are shown in Figure 3.18. Similarly to the initial tests, the lowest ratios are obtained for the cases of resonant monopoles. For the $1 \mathrm{GHz}$ frequency, this behavior overlaps with the $\chi^{2}$ results. Although no apparent rejection rate change is visible in the vicinity of the $2.5 \mathrm{GHz}$ resonance (possibly masked by the sensitivity of the $\chi^{2}$ test), the corresponding max/avg ratio is obviously affected, but to a smaller degree than the $1 \mathrm{GHz}$ resonance. The repeatability of both resonances is validated by the similar behavior of the $99^{\text {th }}$ percentile traces. The shown max/avg is the maximum value of all the ratios in the $60 \mathrm{MHz}$ band. In case of a resonant monopole length, a steep change in the frequency characteristic is present, and although normalized to the corresponding average value, the local max/avg exactly at the resonance might be masked by the higher ratios just around it, which leads to an overestimation of the result shown. Outside of the resonance, a very strong increase of the max/avg is observed, especially in the case of short monopoles. The highest ratios are obtained for the $1 \mathrm{GHz}$ frequency, peaking at almost $19 \mathrm{~dB}$. The 2.5 $\mathrm{GHz}$ results are lower, but still above $15 \mathrm{~dB}$ for these antenna lengths. On the other hand, the maximum results of monopoles longer than $100 \mathrm{~mm}$ converge at around $14 \mathrm{~dB}$, although a clear difference in the $99^{\text {th }}$ is present. Assuming that the difference between the $99^{\text {th }}$ percentile and the total max/avg is a result of limited data, it is crucial to mention that as opposed to the matched antenna cases (also analyzed in Section 3.3.2), loading the chamber with an unmatched antenna does affect the max/avg ratio. 


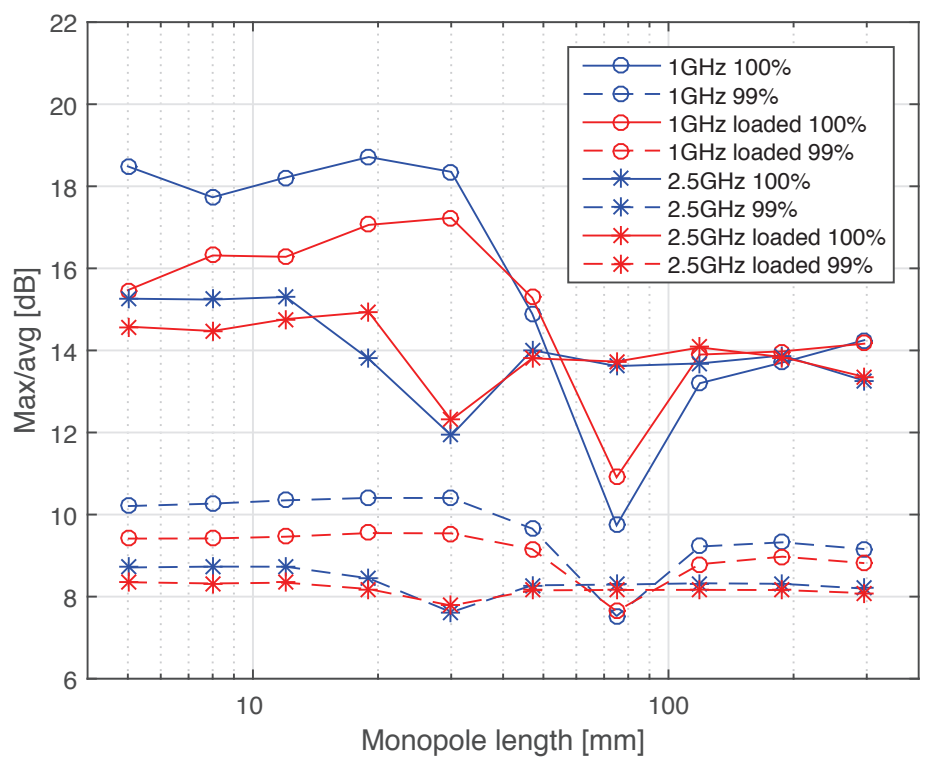

Figure 3.18: Max/avg results for different monopole lengths (SA setup), for different percentiles.

The histograms of the data representing the three most critical points, i.e. the shortest, the resonant, and the longest monopole, obtained in the unloaded chamber at $1 \mathrm{GHz}$ frequency are shown in Figure 3.19. The normalized maximum measured value is marked in Figure 3.19a to help realize the significance of the possible threat. As concluded in [6o], a Weibull PDF is fitted to the data and marked in the same figures. A good fit is obtained not only for the short antenna, but for the long one too, where the skew is also present. In case of a resonant monopole length, the Weibull distribution resembles Rayleigh, and both fit the data decently. Following the results from Figure 3.17, it can be stated that the Weibull distribution is preferred for all lengths except for the resonant case.

The shown results are comparable to the values obtained in the previous section, proving a proper operation of all setups, and validating the observed effects. In almost all cases in this chapter, the data behaved and reacted similarly to the variation of the parameters. The antennas used in the first sections of this chapter were broadband, i.e. matched reasonably well for a wide range of frequencies, and although giving similar results, cannot be directly compared to the cases of matched monopoles with single sharp resonances. It remains unanswered whether the matching or the physical size plays a dominant role in creating the observed phenomena. 


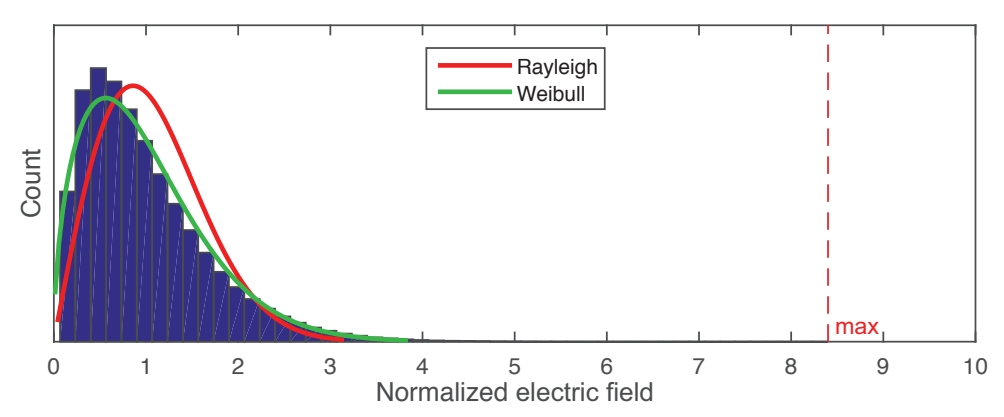

(a) $5 \mathrm{~mm}$ monopole with marked maximum value measured.

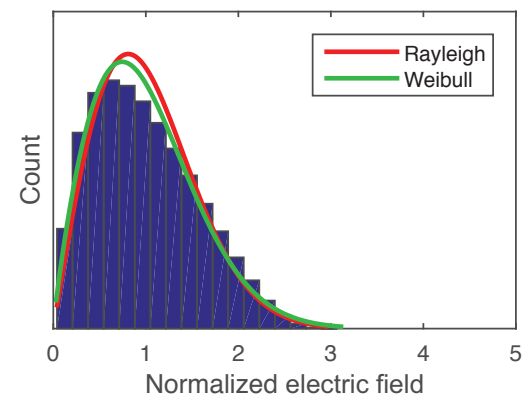

(b) $75 \mathrm{~mm}$ monopole.

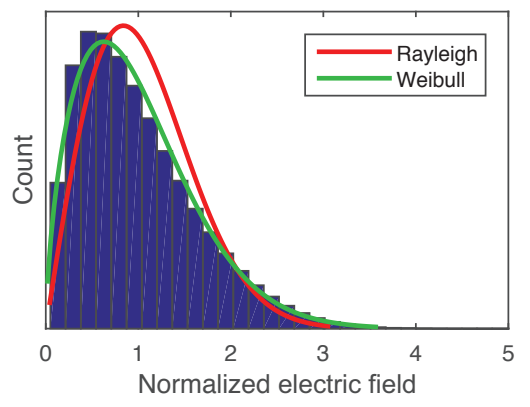

(c) $296 \mathrm{~mm}$ monopole.

Figure 3.19: Histograms obtained using a very short (a), a resonant (b), and a long (c) monopole (SA setup, $1 \mathrm{GHz}$, empty chamber).

\subsection{Summary and discussion}

An empirical analysis of the maximum field strengths has been performed using the setup exploiting the unique capabilities of the VIRC. The unpredictable behavior of the shaking walls supports the generation of new positions and thus more independent samples even after a prolonged time. Using a setup dedicated for recording the envelope of a fast-changing field allowed to observe the creation of strong spikes, i.e. transient occurrences of high electric field values, and their statistical analysis. It has been shown that the prepared setup is capable of continuously delivering the desired independent samples even within a 1-hour measurement, significantly outperforming classical RCs. The technique of performing such long measurements has been used to validate the existing max/avg ratio models by introducing a counter-hypothesis stating that the max/avg ratio is a function of the Q-factor.

The first measurement campaign resulted in 24 1-hour datasets, obtained in three highly different configurations with significantly varying Q-factors 
(altered by means of absorber loading, physical size, and electrical size, i.e. frequency), mode densities, and amounts of independent samples. It has been shown that under most analyzed conditions, the Q-factor does not affect the max/avg ratio, thus rejecting the tested hypothesis.

However, triggered by an outlier in the results obtained, another investigation was performed, aiming at the analysis of max/avg behavior when differently sized receiving antennas are used. The measurements were performed with two entirely different setups, and utilizing different techniques, although both performed in the VIRC. The field distributions and their max/avg ratios were analyzed using the data obtained from measurements performed with 15 different monopoles, long, tuned, as well as in small monopole regime, at two frequency points, and under two loading conditions. It has been shown that the antenna size has a significant impact on the received data distribution. In case of a highly overmoded chamber by means of large volume or high frequency (high mode density) or low Q-factor (high mode bandwidth), the data recorded using a matched antenna fits the Rayleigh distribution very well, giving a consistent max/avg ratio of around 12-13 $\mathrm{dB}$. However, in cases of unmatched monopoles, the recorded data leans towards the Weibull distribution, with significantly increased max/avg ratios. This effect is especially apparent for short monopoles, reaching up to $19 \mathrm{~dB}$, but is also noticeable for long antennas. The effect became less apparent at the higher frequency, giving a $14 \mathrm{~dB}$ max/avg. Furthermore, in the cases of antenna mismatch, a dependency on the Q-factor is evident. The Weibull distributed data becomes Rayleigh if the Q-factor is lowered, which is expected in actual REs. The final judgement of the initial hypothesis regarding the max/avg and Q-factor dependence remains unresolved and requires further research. Additional work should also include a similar analysis of the transmitting antenna effects.

Despite the very high repeatability of the results shown, it is not entirely clear how the antenna size affects the obtainable maximum. A mismatch at the antenna port increases the reflection coefficient, resulting in decreased received power. However, the coefficient is the same for any value, whether it is around the average or a maximum. On the other hand, an increased mismatch could be related to the enhanced backscatter effect [64].

Even without the theoretical explanation, the empirically obtained results remain practically usable, even as warning sign regarding the possible EMI threats in REs. The dimensions of an EUT and especially its critical coupling interfaces should be considered. Additionally, the shown results are applicable to field probes which usually have short monopole antennas, which can lead to higher maximum measured field values when compared to a matched antenna, e.g. as used in RC validation according to [2]. 


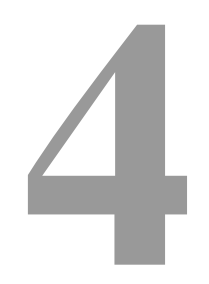

\section{Radiated emissions in reverberant environments}

\subsection{Introduction}

Radiated emission measurements are normally performed in accredited EMC laboratory facilities, such as an open area test site (OATS), semi-anechoic chamber (SAC), or RC. OATS and SAC are designed to mimic free space conditions, with an extra ground reflection, while RCs utilize the multipath and resonant properties of the environment, and deal with them in a statistical way. Those two kinds of solutions represent two opposing extreme approaches of managing reflections - either by eliminating, or by boosting them. However, certain large installations cannot be brought to an EMC chamber and measurements have to be performed on site. Those sites usually do not belong to any of the aforementioned facilities, and their reverberant properties are usually unknown and lie somewhere between free space and multipath conditions. One option could be to bring a VIRC to the test site [65]. The conventional approach used by many manufacturers of large apparatus is to perform EMI measurements on-site at many places around it, and assuming free space conditions, due to the lack of any alternative. However, the presence of reflections can cause errors in estimating the line of sight component, even if it is dominant over the scattered components [66]. Additionally, due to the many 
carefully selected measurement positions, it is also a very time-consuming activity. The access to those points can also be limited by other equipment present at the test site. It has been shown that semi-enclosed environments, e.g. ships, airplanes, or factories, create multipath environments similar in behavior to those of RCs [6], [66], [67], also addressed in Chapter 2 of this thesis. On the other hand, the standard on-site EMC testing, according to [33], is performed by using methods based on OATS or SAC, utilizing the line of sight component. In this chapter, the classification of REs is analyzed in order to find the most suitable solutions for performing on-site emissions testing.

\subsection{Site classification}

A simplified site classification has been introduced in Table 2.1 in Chapter 2, with only two opposite extremes defined in terms of reflection utilization, namely an AC and an RC. As concluded in that chapter, typical industrial REs do not belong to the RC category, although display possibly high reverberant capabilities. For the same reason, they cannot belong to the AC category. Furthermore, it is difficult or sometimes even impossible to validate the industrial sites according to RC or AC standards because of their differences from laboratory conditions. However, the "grey area" unclassified into any of the aforementioned categories can be split into subcategories, depending on their reverberant properties, e.g. Q-factors or IL, as shown in Table 4.1.

The two introduced categories, anechoic test site (ATS) and scattering test site (STS), are briefly compared to their corresponding standardized equivalents in the following sections.

\subsubsection{AC and ATS}

An AC is a Faraday cage, whose walls are lined with absorbing materials attenuating any reflections, thus minimizing the presence of standing waves affecting the field uniformity (used as a validation technique [68]), as well isolating the environment from the outside noise. The very high effectiveness of the absorbers, typically providing reflectivity of around $-40 \mathrm{~dB}$, allows to obtain conditions similar to free space. In EMC, SACs with reflective floor are used to mimic the original OATS conditions, allowing for a single, controlled reflection. The emission measurement is performed by rotating the EUT acting as a transmitting apparatus (Tx) directly in front of the receiving antenna ( $\mathrm{Rx})$ at a given distance, and measuring the LOS and the ground reflected components. This is equivalent to moving the $\mathrm{Rx}$ in a circle around the $\mathrm{Tx}$, as shown in Figure 4.1. At each position, the elevation of the $\mathrm{Rx}$ is additionally changed to find the peak (constructive interference) of the summed rays. The measured 
Table 4.1: Extended site classification.

\begin{tabular}{|c|c|c|c|}
\hline \multicolumn{2}{|c|}{$\leftarrow$ Lower $\mathbf{Q}$} & \multicolumn{2}{|c|}{ Higher $Q \rightarrow$} \\
\hline \multicolumn{2}{|c|}{ Q threshold } & Q threshold & Q threshold \\
\hline $\begin{array}{c}\text { Anechoic Chamber } \\
\text { (AC) }\end{array}$ & $\begin{array}{c}\text { Anechoic Test Site } \\
\text { (ATS) }\end{array}$ & $\begin{array}{l}\text { Scattering Test Site } \\
\text { (STS) }\end{array}$ & $\begin{array}{l}\text { Reverberation } \\
\text { Chamber (RC) }\end{array}$ \\
\hline $\begin{array}{l}\text { - Fulfills the } \\
\text { requirements from } \\
\text { an AC standard. } \\
\text { - Measurement } \\
\text { procedure } \\
\text { according to the AC } \\
\text { standard. }\end{array}$ & $\begin{array}{l}\text { - Possibly fulfills } \\
\text { the requirements } \\
\text { from an AC } \\
\text { standard. } \\
\text { - Does not fulfill the } \\
\text { requirements from } \\
\text { an RC standard. } \\
\text { - Environment not } \\
\text { reflective enough } \\
\text { for proper } \\
\text { multipath } \\
\text { operation. } \\
\text { - Measurement } \\
\text { procedure based on } \\
\text { the AC standard } \\
\text { (direct illumination, } \\
\text { line of sight). } \\
\text { - Result correction } \\
\text { and error } \\
\text { estimation depends } \\
\text { on the deviation } \\
\text { from the AC } \\
\text { standard. }\end{array}$ & $\begin{array}{l}\text { - Almost fulfills the } \\
\text { requirements from } \\
\text { an RC standard. } \\
\text { - Does not fulfill the } \\
\text { requirements from } \\
\text { an AC standard. } \\
\text { - Environment } \\
\text { reflective enough } \\
\text { for proper } \\
\text { multipath } \\
\text { operation. } \\
\text { - Measurement } \\
\text { procedure based on } \\
\text { the RC standard } \\
\text { (multipath, no line } \\
\text { of sight). } \\
\text { - Result correction } \\
\text { and error } \\
\text { estimation depends } \\
\text { on the deviation } \\
\text { from the RC } \\
\text { standard. }\end{array}$ & $\begin{array}{l}\text { - Fulfills the } \\
\text { requirements from } \\
\text { an RC standard. } \\
\text { - Measurement } \\
\text { procedure according } \\
\text { to the RC standard. }\end{array}$ \\
\hline
\end{tabular}


values depend on the radiation pattern of the EUT, therefore the measurement is performed for multiple angles. Although time-consuming, this method allows to perform measurements with a presumably high repeatability because of the assumed deterministic nature of the test setup.

In case of a real, imperfectly anechoic environment, stronger reflections from the surrounding walls or objects are expected, and the floor is not necessarily reflective. Due to other objects present on site or the vicinity of the walls near the immovable apparatus, it is not granted that every position around it is available. Applying the very same method of performing the measurement as in the AC but only at single positions, as recommended by [33], can result in very high errors [66]. The field received by the $R x$ is a summation of the deterministic LOS component with components scattered unpredictably from the surroundings as shown in Figure 4.2, expected to come from the Rice distribution [69]. In case of using high gain Rx antenna, as well as poor reverberant properties of the site, the k-factor is high, and the distribution resembles Gaussian (with the mean around the value of the LOS component). In order to separate the desired LOS components, it is here suggested to introduce a form of averaging, e.g. volume sampling or using a portable stirrer. In the former case, due to the dependency of the LOS component on the radiation characteristic of the apparatus, it is crucial to sample the far field along the line starting in its middle, i.e. changing distance only. Similarly to small scale fading in radio communications, approximating the data with an exponential curve, using even a very low amount of measurement points, can significantly lower the error with respect to a single measurement. Further approximations, also related to the limited amount of measurement angles and the estimation of the radiation pattern, can be made using the unintentional radiator principle [70]. However, due to the high amount of simplifications and mistake possibilities, the modification suggested here requires more attention and deeper analysis. It is worth mentioning that the big advantage of the ATS method is the utilization of the strong LOS component, as opposed to only using scattered components, used in the STS described in the next section, that are more prone to disappear below the noise floor.

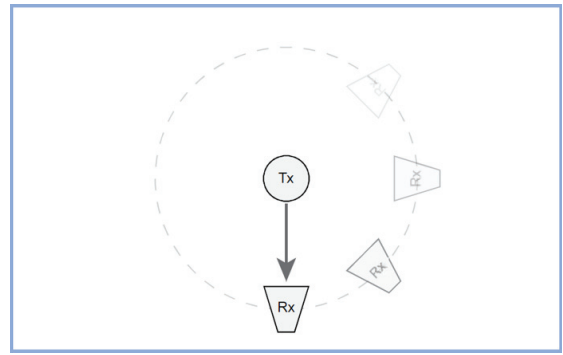

Figure 4.1: Anechoic chamber.

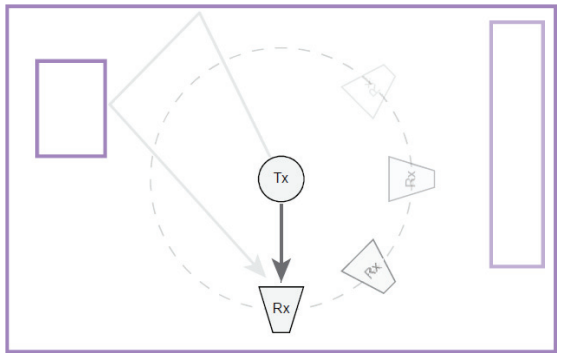

Figure 4.2: Anechoic test site. 


\subsubsection{RC and STS}

In contrast to an $\mathrm{AC}$, an $\mathrm{RC}$ is a Faraday cage with highly reflective walls, maximizing the strength of reflections, creating a resonating field. As already described in Chapter 1 and 2, due to the amount of reflections, field complexity, and high sensitivity to any changes in the boundary conditions causes the field parameters to be estimated in a statistical way. The data necessary for statistical analysis is typically collected by altering the modal structure by changing the boundary conditions using a mechanical stirrer [2], as shown in Figure 4.3. Removing the unstirred components, e.g. by orienting the directional $\mathrm{Rx}$ antenna away from the source, the radiation pattern of the measured apparatus becomes irrelevant in the isotropic and statistically uniform field, therefore its position in the working volume is arbitrary. The high statistical repeatability of the collected data ensures proper estimation of the TRP.

As described in Chapter 2, an imperfect industrial RE can resemble an RC in terms of managing reflections, thus becoming an STS. Although strong deviations are possible, the emission measurement technique can be applied in an almost unchanged state. Due to no LOS component measurement, the volume sampling method can be used by placing the $\mathrm{Rx}$ antenna in almost any available spatial point, avoiding the other neighboring objects, as shown in Figure 4.4. Although the environment is not expected to always provide a good fit to the theoretical data distributions (as shown in Figure 2.19 in Chapter 2), the statistical repeatability of the data recorded using a reference transmitter used for calibration, and the actual apparatus is expected to be reasonably high. The major concern lies in the potentially low power of the scattered field, possibly being masked by the ambient noise in the unshielded environment, even in case of strong direct radiation.

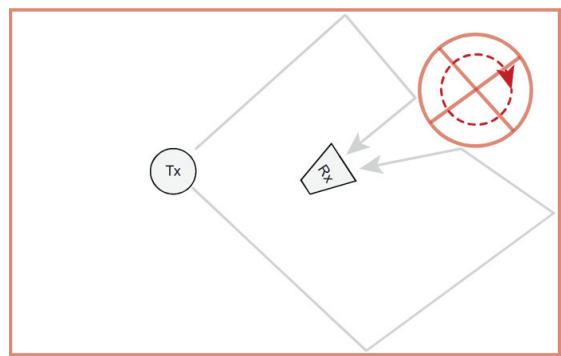

Figure 4.3: Reverberation chamber.

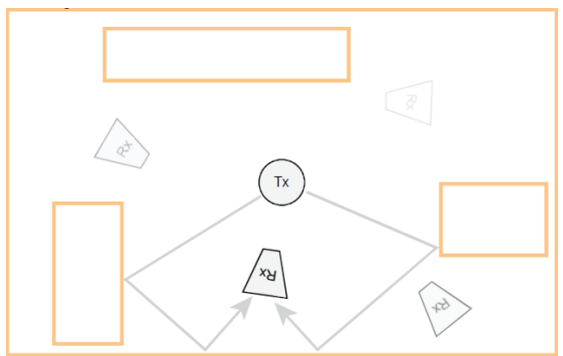

Figure 4.4: Scattering test site. 


\subsection{Experimental STS evaluation}

From the two categories introduced in the previous section, usable for performing emission tests of immovable objects, ATS and STS, the second one was selected as more efficient and promising, as well as better fitting the scope of this thesis. Therefore, in this section, the STS method is evaluated experimentally. When dealing with environments operating outside of overmoded conditions typical for RCs, it is difficult to utilize the theoretical probability distribution functions based on perfect multipath models. Due to the high spread of the quality factors of the excited modes, the distributions of recorded samples can vary significantly. However, the standard radiated emission measurement technique uses only single values, averages or maximums of the collected data. As long as the repeatability of those is reasonably high and the uncertainties in their estimations are low, the test can still be performed successfully, even if the field distribution is far from the perfect RC. In this section, the measurement errors and uncertainties when performing measurements on site rather than in a standard RC are investigated. The tests are based on the IEC 61000 4-21 radiated emissions technique [2]. Measurements were performed in two different industrial environments, previously analyzed in Chapter 2: a typical office and an industrial workshop. A comb generator was used as an example apparatus. All the results obtained on-site are compared with the ones obtained in a standard RC. The uncertainties were calculated by applying a simplified, but generalized method, applicable to imperfect environments, critical for making the decision about passing the emission test. The major contributors to the uncertainties are traced and discussed. Furthermore, the results are compared to the current on-site emission measurement standard by using the unintentional antenna model [71]. Eventually, a test site IL threshold is defined for apparatus of different sizes.

\subsubsection{Setup description}

The emission measurements were performed in a manner similar to RE analysis from Chapter 2. This time though, the transmitting antenna was replaced by a comb generator acting as an actual, immovable apparatus, placed in a fixed position. The previously transmitting discone antenna was always present, terminated with a 50 Ohm load. A receiving PICA antenna was connected directly to a portable SA, working in an EMI receiver mode with a $120 \mathrm{kHz}$ resolution bandwidth (RBW) filter. Twenty one (21) frequency points overlapping with the comb generator peaks were uniformly selected in the frequency range between $300 \mathrm{MHz}$ and $1.3 \mathrm{GHz}$. The samples necessary for the statistical processing were obtained using the same volume sampling technique as described in the RE analysis, giving 50 independent power 
measurements per frequency point. Additionally, the noise floor levels were recorded with the comb generator turned off.

\subsubsection{Results}

The data recorded according to the methods described in the previous section are presented in Figure 4.5. Obviously, the average and maximum received power are the highest in case of a high Q-factor $\mathrm{RC}$, and is always at least $20 \mathrm{~dB}$ over the average $-85 \mathrm{dBm}$ noise floor. The power levels recorded in the other two environments are significantly lower and thus become more affected by the vicinity of the noise. The TRP calculation is based on [2], although, as the analyzed case is not a calibrated RC, it has been modified and optimized for use in imperfect REs. In [2], it is recommended to use the average values due to high repeatability and accuracy, although the usage of maximum readings is also possible. The latter method requires calculation of the parameters obtained in the unloaded chamber, which is impossible in case of immovable apparatus. However, assuming that the max/avg ratio does not change between unloaded and mildly loaded conditions, as concluded in Chapter 3, it can be shown that the TRP can also be calculated this way to benefit from increased measurement sensitivity and thus decreased error associated with low signal-to-noise ratio (SNR). In this work, both approaches are used and compared. As opposed to the maximum power, being just a single highest result, with at least a couple of $\mathrm{dB}$ above the noise, the average power is calculated from all the 50 measured points, most of which are very close to the noise. The TRP can be calculated using the equations 4.1 and 4.2, depending on whether the maximum $P_{\max }$ (with minimum insertion loss $\mathrm{IL}_{\text {min }}$ ) or average $P_{\text {avg }}$ power (with average insertion loss $\mathrm{IL}_{\mathrm{avg}}$ ) is used.

$$
\begin{aligned}
& \mathrm{TRP}=\frac{P_{\max } \times \mathrm{IL}_{\min }}{\eta_{R x}} \\
& \mathrm{TRP}=\frac{P_{\mathrm{avg}} \times \mathrm{IL}_{\mathrm{avg}}}{\eta_{R x}}
\end{aligned}
$$

where $\eta_{R x}$ is the total efficiency of the receiving antenna.

Each result is calculated using a single interpolated IL value between the two points closest to the frequency of interest. The total radiated efficiencies incorporate measured mismatch losses, while the radiation efficiencies of the antennas were assumed to be 0.9, based on [2]. The results are shown in Figure 4.6. 


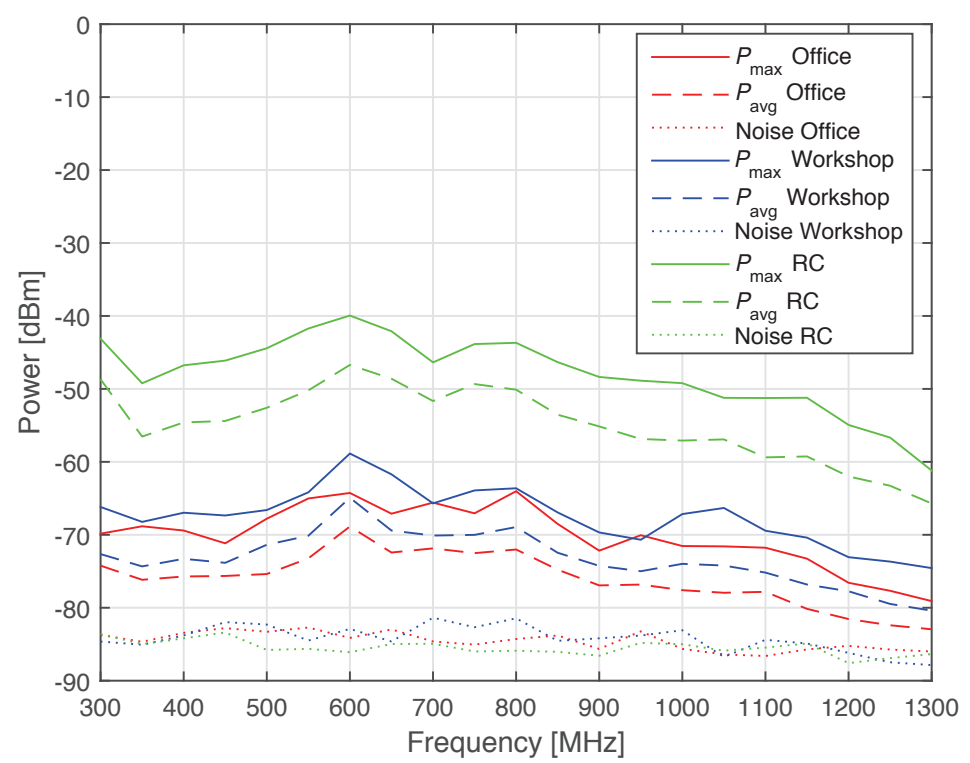

Figure 4.5: Raw maximum and average received power measurements, as well as the noise floors of the setups, before applying corrections.

\subsubsection{Error and uncertainty estimation}

As mentioned in the IEC 61000-4-21 standard [2], the highest measurement accuracy is reached when the average readings (both IL and raw power) are used. Also, in [38], it is stated that the uncertainties of such emission measurements performed in a properly operating RC are very low, around 1-2 $\mathrm{dB}$. Therefore, the TRP calculated using the averages in RC is considered to be the reference level for the calculation of the error performed in the workshop and office environments. This error, expressed as a ratio of the TRP results, is shown in Figure 4.7. It is very important to note that the error is mainly positive and grows with frequency up to around 10-15 $\mathrm{dB}$ beyond $1 \mathrm{GHz}$. The error is also higher in the office environment than in the workshop.

In this section, it is analyzed whether the main contribution to this error comes from the poor reverberation, resulting in low statistical field uniformity and repeatability of statistics, or perhaps from the vicinity of the noise floor and imperfections of the measurement equipment. 


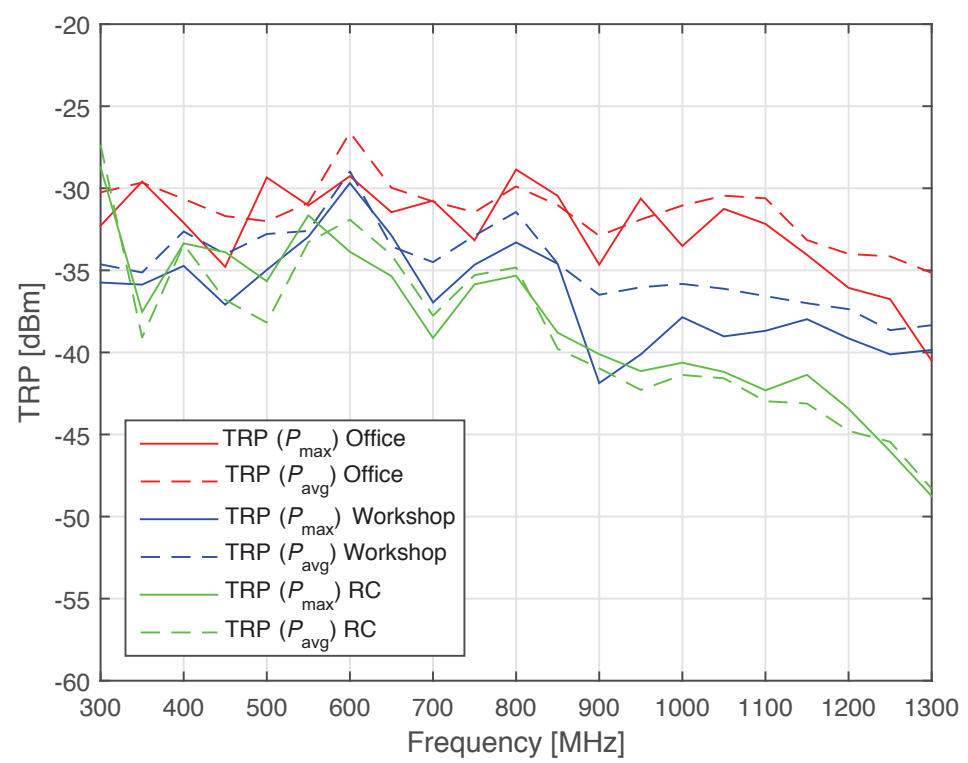

Figure 4.6: Total radiated power results.

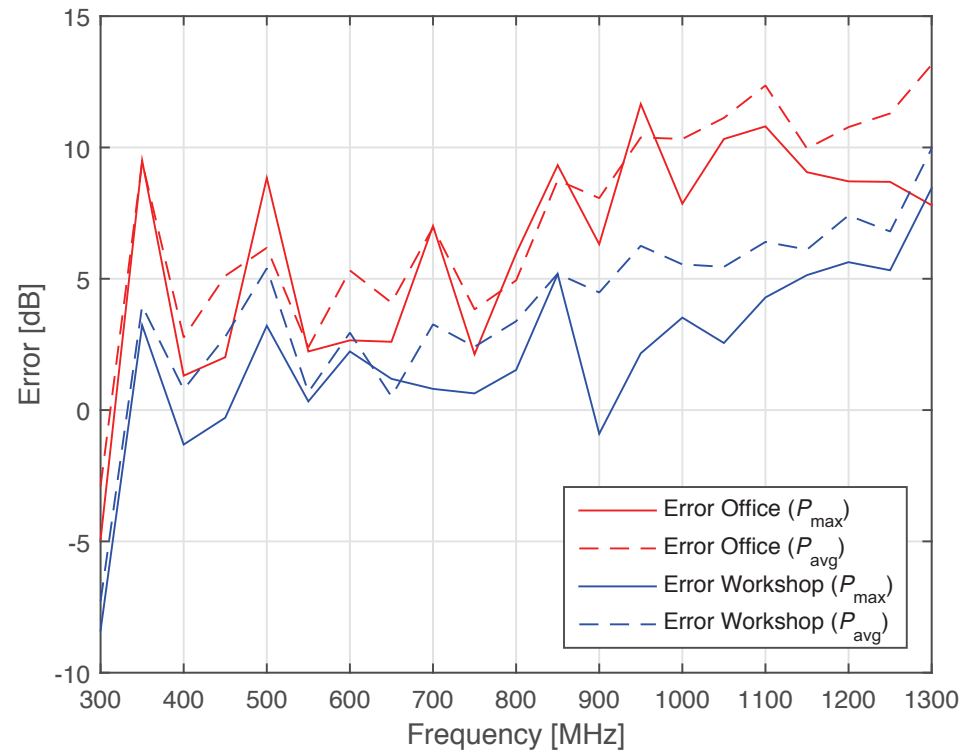

Figure 4.7: Errors in office and workshop with respect to the RC results. 


\section{Field uniformity}

Although comparable, but inconsistent similarity of REs and RCs, as concluded in Chapter 2, the uncertainty analysis presented here does not utilize the theoretical model of a perfect RC. The presented analysis is entirely empirical, and although the obtained uncertainty levels may be overestimated and serious simplifications are made, it is applicable to any environment, or as a worst case scenario. No assumptions regarding the distribution of measured samples are made. Only independent distributions of averages and maximums are considered.

To properly estimate the emission levels, the statistical field uniformity is the most important parameter. The repeatability of behavior in measuring the calibration data, IL, and the received raw power levels ensure proper estimation of the TRP.

The estimations of the true means and obtainable maximums are both functions of number of recorded samples [54]. However, even if this distribution is unknown, the distributions of its parameters can be anticipated. According to the CLT, the average values come from a Gaussian distribution, while the obtainable maximums come from a GEV distribution [48]. The GEV distribution can be similar in shape to the Gaussian distribution, especially when comparing the data histograms consisting of a low amount of samples. It is the case in this work, therefore the distribution of the maximums is simplified to be Gaussian. This simplification does not cause an error higher than the error associated to the distribution fitting and resulting estimation of the intervals. The obtained data also does not fail the GOF test for normality, supporting this decision. This simplification allows to use symmetrical confidence intervals.

Each set of 50 measurements gives only a single maximum and a single average, but in order to perform a statistical analysis, more samples can be obtained by applying the frequency stirring technique [66]. It is commonly used in RC, and allows to increase the amount of obtainable samples. The frequency stirring was performed in a bandwidth of $50 \mathrm{MHz}$. The frequency separation of two uncorrelated points is $\Delta f>f / Q$ [66], which in case of very low Q-factors in office and workshop environments, is at least $10 \mathrm{MHz}$ and $5 \mathrm{MHz}$, respectively. However, in order to increase the very low number of samples to a reasonable amount, the data was sampled every $2.5 \mathrm{MHz}$, giving 20 points per frequency stirring band. Although it seems to be a rather drastic violation, it is shown later that, in fact, it is not. From each set of 20 samples, the upper and lower 95\% confidence intervals (CI) and prediction intervals (PI) can be calculated using the t-Student distribution [72].

The frequency stirring technique is based on the assumption of ergodicity around the frequency of interest, which is commonly met in case of over- 
moded, passive chambers. In the analyzed case, where the parent distribution might change due to high Q-factor variations, additional simplifications are necessary. Assuming that the points at neighboring frequencies come from similar distributions, but with different scale factors due to differences in the composite Q-factors, the IL data were first normalized to a smoothed mean using a $50 \mathrm{MHz}$ moving average filter to calculate the standard deviations in each frequency stirring band. The obtained standard deviations were then normalized to the mean of each band. Not performing such a normalization greatly increases their overestimation. The result of such an operation is depicted in Figure 4.8.

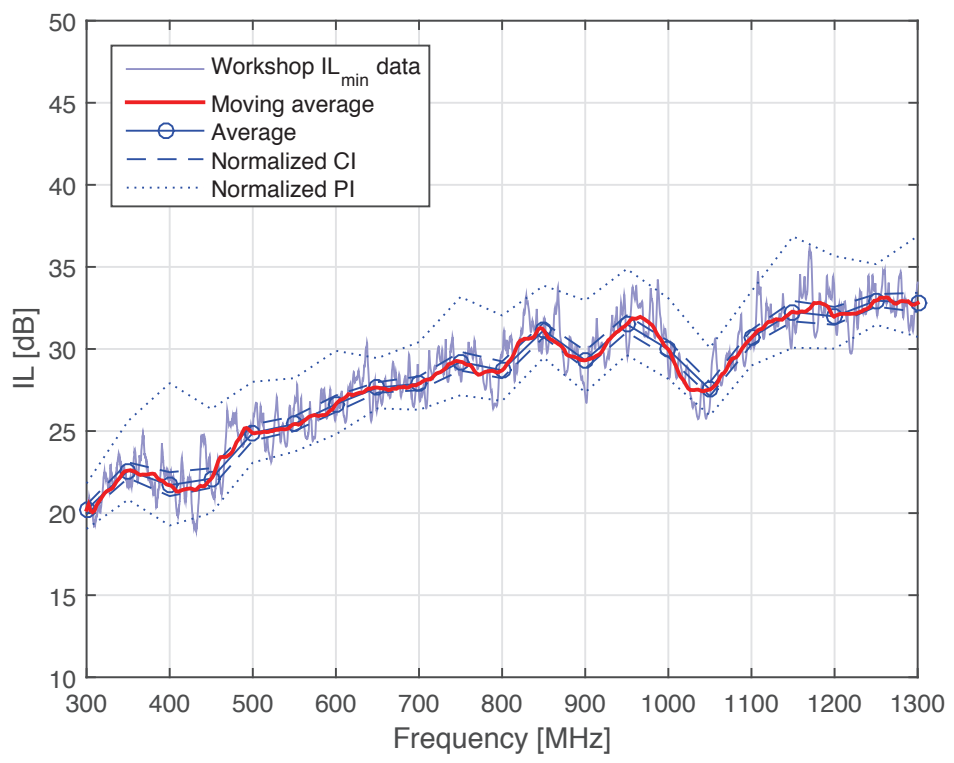

Figure 4.8: IL data is first normalized to the smoothed (red) trace to calculate the standard deviation before calculating the intervals.

In a chamber with a certain Q-factor, it can be shown that the standard deviation of the obtained distribution, e.g. of averages, is proportional to its mean. Therefore, both the CI and PI calculated from the IL data can be scaled to any other value recorded in the same environment. It was additionally observed that the normalized standard deviations, and therefore CI and PI normalized to their means, are almost identical in all three environments, and also in the whole analyzed frequency range. Averaging the normalized intervals allowed then to find a single CI and PI, scalable to any level. Averaged and normalized intervals somewhat neglect the breach related to previously mentioned possible oversampling. It is difficult to talk about statistics of a 
single power point (average or maximum), but it is possible to guesstimate the CI of means of distributions (of averages or maximums) that the sample could possibly come from. Knowing that a PI defines the boundaries around the average where the next point could be with a $95 \%$ confidence, it can also be stated that the means of two extreme possible distributions, from which that point might come, lie within the $\pm|C I+P I|$ range from that point. If that point is an actual power measurement average or maximum, and CI and PI normalized to that point are used, it is possible to estimate an interval around that point where $95 \%$ of possible means are. This guesstimation is depicted in Figure 4.9. Furthermore, the TRP calculation from 4.1 and 4.2 include values with both uncertainties: related to unknown distributions of power points $P_{\max }$ and $P_{\mathrm{avg}}$, as well as the $\mathrm{IL}_{\min }$ and $\mathrm{IL}_{\mathrm{avg}}$ uncertainty. The total TRP uncertainty due to field uniformity was calculated using a root sum squares error propagation method, combining all uncertainties calculated from intervals normalized to appropriate averages, as shown in Figure 4.9.

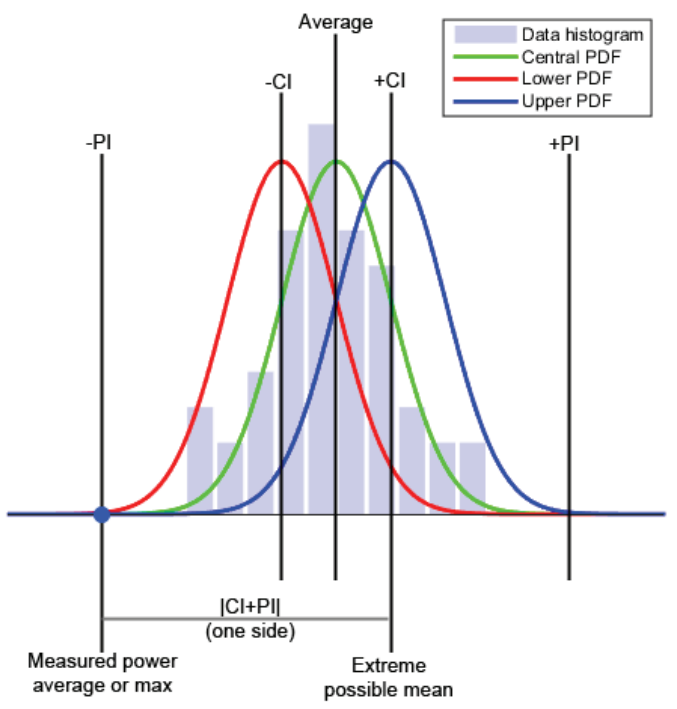

Figure 4.9: Single power point average or maximum belongs a distribution with the mean up to $\pm|\mathrm{CI}+\mathrm{PI}|$ (normalized to the average) away from it. 


\section{Noise and nonlinearities}

Low SNR is known to have a potentially devastating effect on any measurement results. It is possible to eliminate the influence of the noise from the relative measurements, such as $S_{21}$ and resulting IL, simply by increasing the output power, but it is an inevitable factor playing a major role in the on-site emission measurements, especially with no LOS component present. The error associated with low SNR can vary significantly and depends on the parameters of the receiver, particularly the RBW filter, the detector type, and the inherent noise floor of the equipment. The requirements regarding the filter and detector for EMI measuring receivers are optimized for laboratory environments and might not be suitable for external testing. Additionally, proper, high quality measurement equipment is usually not compact enough, therefore portable receivers are preferred. Unfortunately, the latter ones often might not provide the required quality, have an elevated noise floor, and could bottleneck the discussed method. Additionally, the imperfect equipment can introduce harmful nonlinearities, especially if a preamplifier is used, which is often the case in low SNR measurements.

The magnitude of this error was estimated by performing a measurement by directly connecting the output of a generator to the input of the used receiver with the same settings as during the actual emission measurement, at a constant frequency of $800 \mathrm{MHz}$. The results shown in Figure 4.10 indicate that the error represented as the difference between output signal power and measured power increases for low powers. Such a correction deals with three factors: equipment noise presence, receiver nonlinearity, and cable loss. Note that this correction does not take care of the ambient noise possibly present at the test site (not in this case), which should be additionally corrected for.

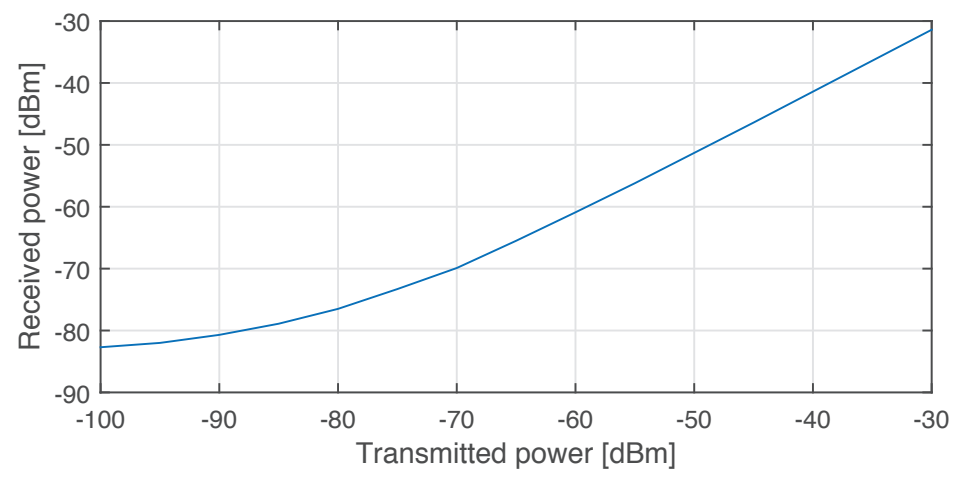

Figure 4.10: The nonlinearity of the receiver in the vicinity of the internal noise floor, measured at the center of the analyzed frequency band, $800 \mathrm{MHz}$. 


\section{Corrected results}

The total radiated power results along with their uncertainties calculated in the previous section, with incorporated all mentioned receiver corrections, are presented in Figures 4.11 and 4.12, respectively. A significant improvement with respect to the results shown in Figure 4.7 can be observed in Figure 4.13, lowering the error to $3-4 \mathrm{~dB}$ in case of the workshop, and 5-10 $\mathrm{dB}$ in the office, which is surprisingly decent for on-site measurements.

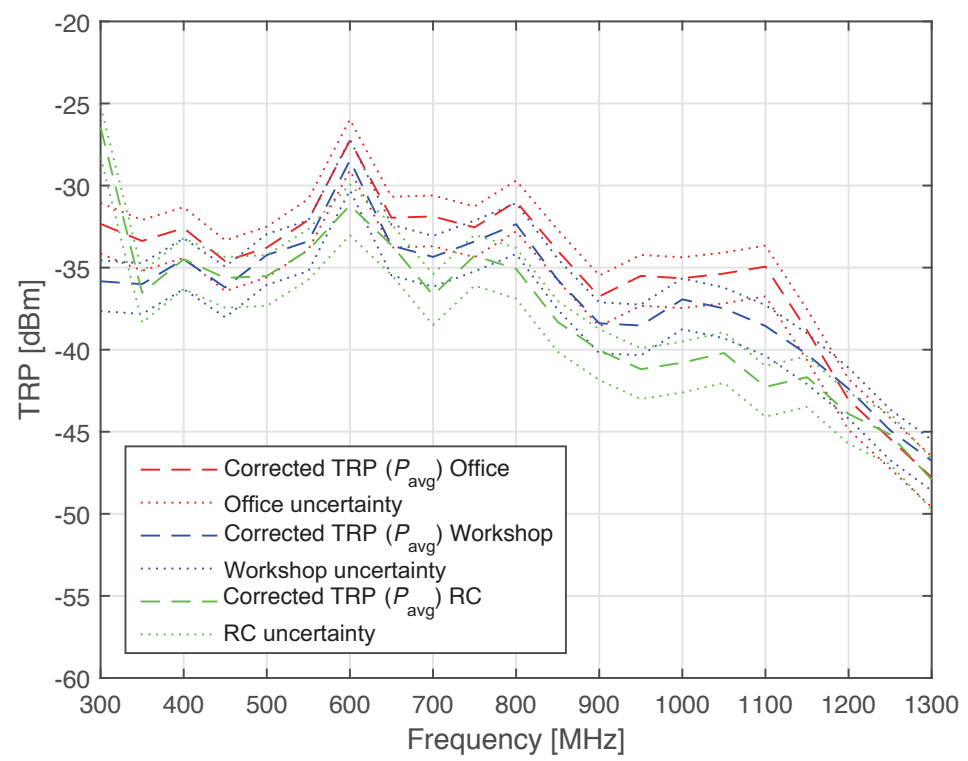

Figure 4.11: TRP calculated from average power readings.

\subsubsection{Insertion loss threshold}

The results shown in this analysis indicate that the total radiated power measurement campaign, performed in the example workshop and office environments, can be successful. In this case, at least the peaks of the measured power shown in Figure 4.5 are above the noise. However, this might not always be the case. It is possible to define an IL threshold, above which the applied measurement technique does not work anymore because the theoretical TRP calculated from the receiving equipment noise floor itself already fails the test by exceeding the limit line. The TRP measurements based on the RC techniques do not deliver the information about the radiation pattern of the EUT. Therefore, in order to compare the TRP against a standard limit line, 


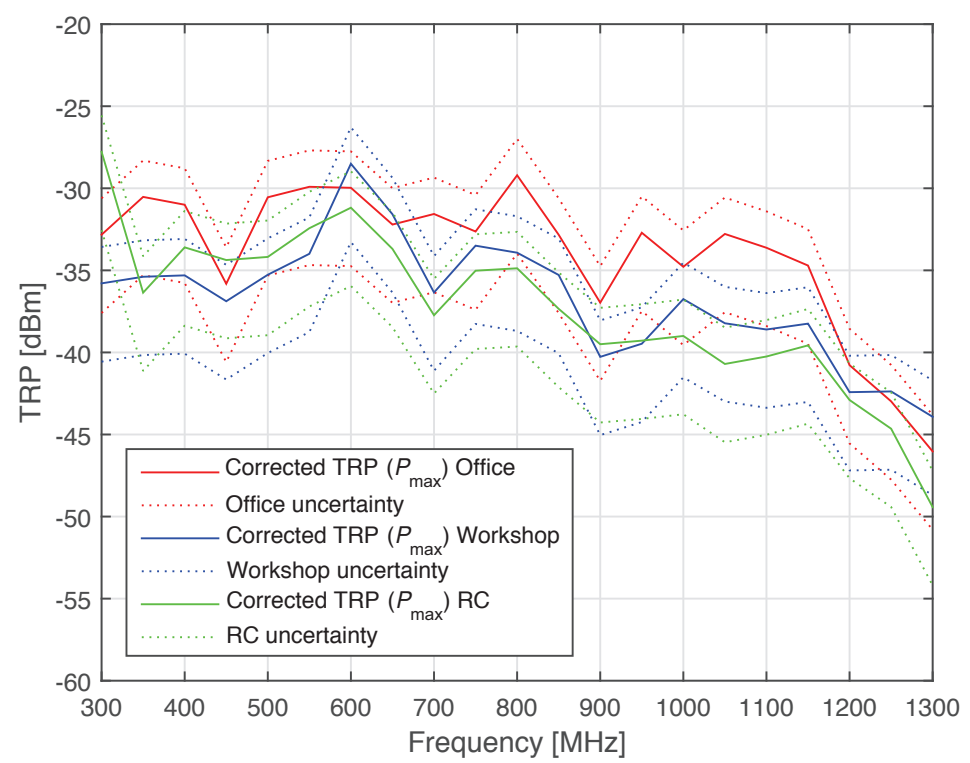

Figure 4.12: TRP calculated from maximum power readings.

defined as a maximum allowed electric field strength at a given distance, an estimation using the unintentional radiator principle [71] can be applied. The results of the emission levels estimated at a $10 \mathrm{~m}$ distance are shown in Figure 4.14 along with the results obtained at an OATS and the IEC 61000-6-4 limit line [33]. From the OATS measurement, it is known that the measured apparatus does not pass the emissions test, which is also the result of the on-site measurements. However, the first important question is whether this method even allows to measure emission levels that would be below the limit line, considering the possibly very low SNR. The sensitivities, i.e. the electric field emissions estimated from the $-85 \mathrm{dBm}$ receiver noise floor itself are shown in Figure 4.15. Unlike the RC, which provides IL low enough to create at least a $20 \mathrm{~dB}$ margin, the workshop, and especially the office site results lie very close to the limit, and even exceed it. Using the results calculated from the maximum values allows to stay under the limit, therefore gives a chance to successfully perform and pass the test, even with wider uncertainty values compared to the average ones. To further generalize this observation, the IL thresholds, for different EUT sizes, are shown in Figure 4.16. Exceeding the shown thresholds renders the STS method immediately unusable, and the ATS method should be used instead. Furthermore, the measurement uncertainty has to be considered before judging if a test is passed or failed. 


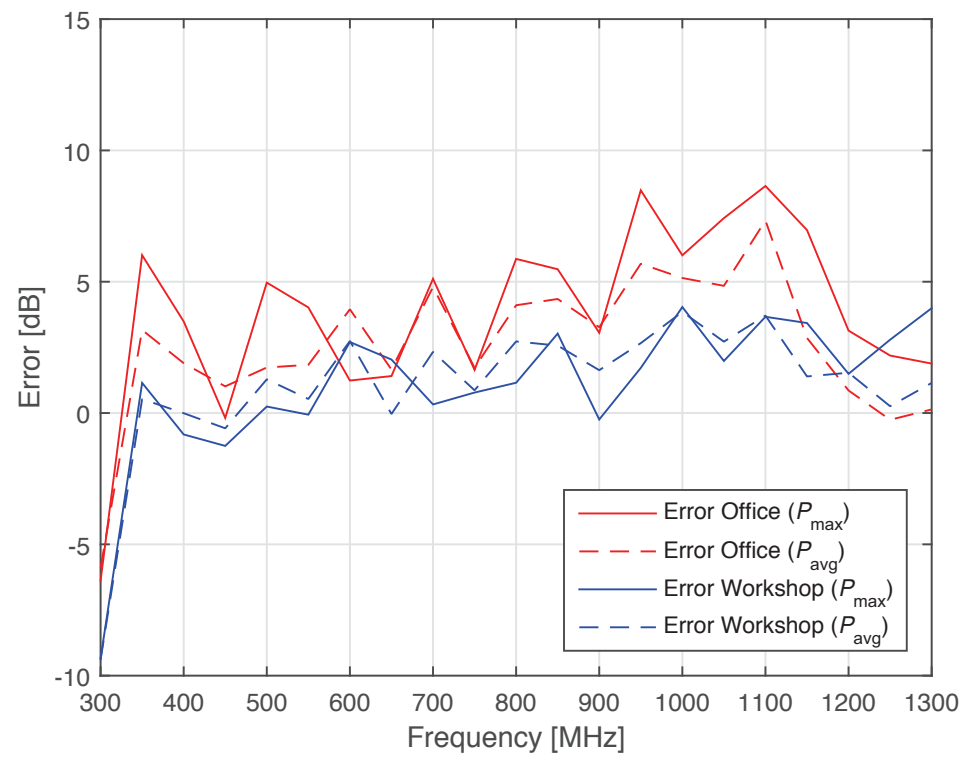

Figure 4.13: Errors in office and workshop with respect to the RC results, with corrections.

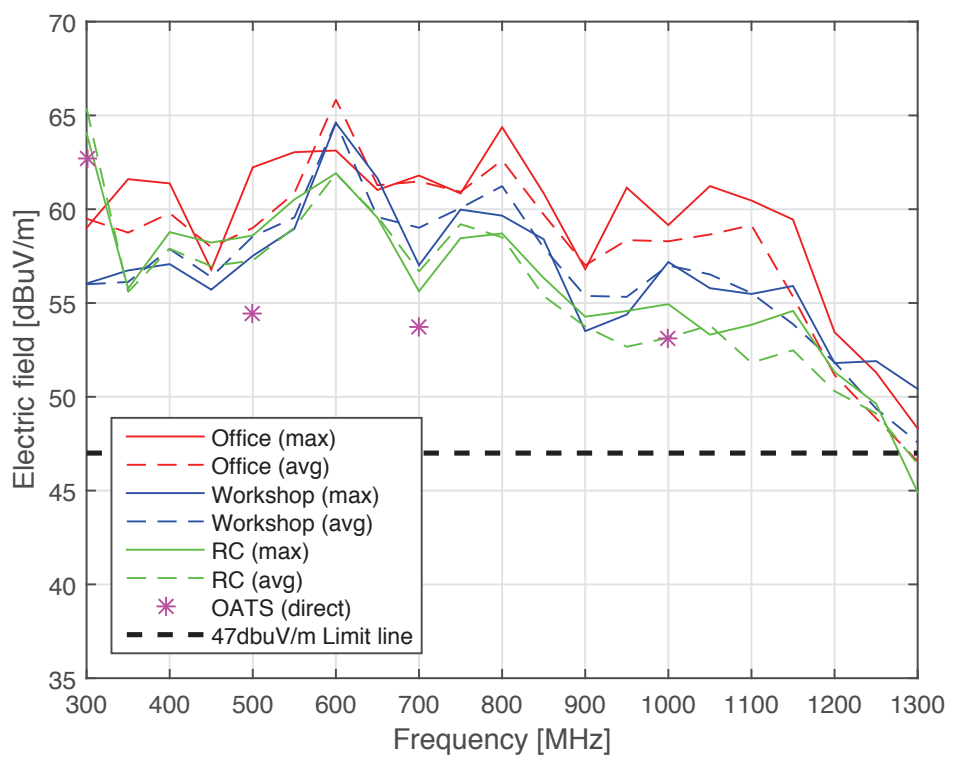

Figure 4.14: Calculated E field from an unintentional radiator at a $10 \mathrm{~m}$ distance. 


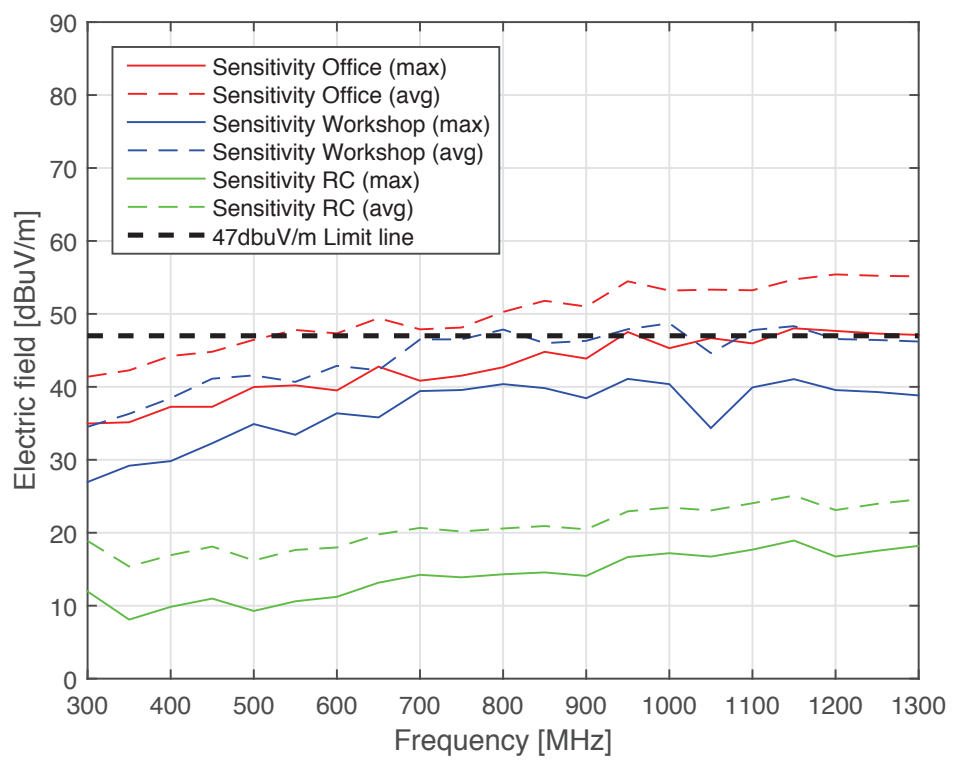

Figure 4.15: Sensitivity (minimum measured E, calculated from the noise floor) and the limit line.

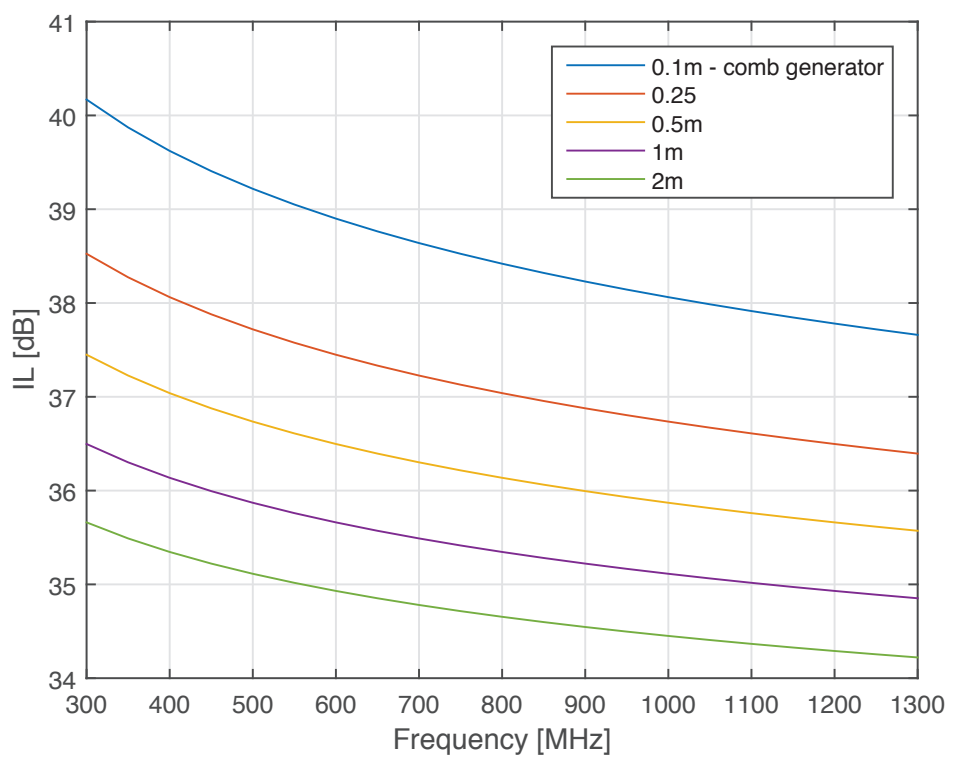

Figure 4.16: Maximum IL to keep $-85 \mathrm{dBm}$ noise floor under the limit line for different sizes of unintentional radiators. 


\subsection{Summary and discussion}

Moving heavy, industrial apparatus to an EMC laboratory can sometimes be impossible or inefficient, therefore the radiated emissions testing has to be performed on-site. Due to the imperfect conditions of those environments, they can be classified as neither (S)AC nor RC, and alternative methods have to be used. The ATS and STS categories introduced in this chapter, allow to improve the measurement accuracy with respect to the currently recommended method [33].

The STS method has been evaluated by performing emission measurements in the imperfect REs. A classical office and a more industrial workshop environment have been used for the experiments. It has been shown that it is possible to perform on-site measurements with an unexpectedly low error. It has been shown that this error can be less than $5 \mathrm{~dB}$ to $10 \mathrm{~dB}$ in the performed experiments. The discussed method is fast and allows to perform emission measurements on a site, even with a very limited space for line of sight measurements, which would normally require much more precise scanning around the apparatus. The IL is a very simple but critical indicator, telling if the emission test can be performed on site. It is also used as a calibration factor, and additionally allows to estimate the uncertainties of the measurements.

The used method of predicting the two extreme distributions of averages or maximums does not depend on the parent distributions, therefore is suitable for imperfect REs. However, it was found that the uncertainties calculated this way, in this analysis, are almost exactly the same in cases of the office, the workshop, and the RC.

Because the intervals of on-site measurements do not always overlap with the presumably correct RC results, it is concluded that there is another source of error, possibly related to the anisotropy of the environment. It has been shown that the correction for the receiver nonlinearity when operating in the vicinity of the noise floor plays a significant role in the calculation of the TRP. This simple measurement can lower the error by around $5 \mathrm{~dB}$ and should always be performed in cases of low SNR. Using maximum readings increases the uncertainty of the measurement, leading to a higher margin when compared with the limit line, but increases the sensitivity of the setup by around 10 $\mathrm{dB}$, thus lowering the error associated with the vicinity of the noise floor.

An IL threshold calculation has been presented, which allows to easily estimate whether the STS method can be used. Otherwise, the ATS method is preferred due to its utilization of the LOS component, which is expected to be stronger than the weak scattered field. 
Moreover, modifications of the test site are possible to improve or degrade its reverberant properties, such as placing metallic plates or RF absorbers in critical locations, depending whether the STS or the ATS method is preferred. Furthermore, a portable VIRC [73] can be used to additionally isolate the apparatus from the outside noise as well as increase the reverberant properties of the setup. 


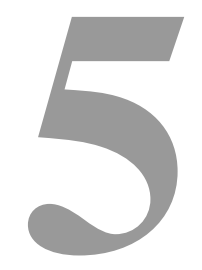

\section{Field-to-cable coupling in reverberant environments}

\subsection{Introduction}

The main objective of EMC is to ensure that all pieces of electronic equipment can operate properly together [74]. The equipment has to go through numerous tests of its emission and immunity levels, most often performed in standardized laboratory environment, following strict rules. It is known from EMI theory that some of the most effective coupling mechanisms between conducted and radiated energy are loops and resonating conductors. So it happens that in real equipment those unwanted antennas can be printed circuit board tracks, grounding loops, wired connections, and even supply cables. As much as the inner, often electrically short lines can successfully be measured/calculated/simulated without significant errors and with decent repeatability, the electrically long connections may become a challenge to analyze due to their highly variable impact on and of the environment. Examples of such cases can be found in modeling complex structures like the cable network in an airplane. The analysis of the electromagnetic field coupling mechanisms to and from single- and multi-conductor transmission lines is a very important aspect of EMC and has been a pursued topic for a rather long time [75]. There are many successful studies performed on plane waves cou- 
pling to uniform (straight) lines [76], which are based on fundamental models, overviewed in [77], although they are not always suitable for real life situations. In the real world, outside of the laboratory, both the electromagnetic fields and transmission line routing can become unknown, random. The exact structure of the exciting field or the geometry of the lines are not deterministic. Furthermore, numerical and analytical solutions of complex systems using the fundamental models would become rather difficult and computationally demanding. Therefore, additional studies have been improving the models by means of statistical analysis to better resemble the real environments, and being optimized for performing calculations within a reasonable time [78]. Random field coupling to uniform transmission lines has been studied in e.g. [79][8o][81][82][83][84]. Further investigation includes coupling of deterministic plane waves into randomly routed (non-uniform) lines [85][86] and the analysis of random field coupling to non-uniform transmission lines [87]. All the aforementioned approaches are shown in Table 5.1 with the corresponding case numbering used throughout this chapter. The plane wave excitation environment was created in a gigahertz transverse electromagnetic (GTEM) cell, and the random field in a VIRC. An issue, common for all numerical and analytical models, is the large amount of variable parameters of varying sensitivity, e.g. radiation resistance [88], leading to a necessity of applying certain simplifications. On the other hand, the experimental approach sacrifices the ability to obtain a large amount of samples required for the statistical study, but includes all the real, rather than assumed, properties.

Most models focus on finding exact and accurate approximations, but is this accuracy always really required? In real life applications, which very often act as REs, the randomness of the field is combined with the randomness of the transmission line geometry, which can then lead to a significant increase of the result dynamics. In EMC, the worst case scenario is usually the desired parameter and the standards are based on general limits and thresholds, often with a hefty error margin. Simplified rules of the thumb are frequently used. This work, being an experimental extension of the work

Table 5.1: General field-to-line coupling cases.

\begin{tabular}{|c|c|c|}
\hline & $\begin{array}{c}\text { Uniform } \\
\text { transmission line }\end{array}$ & $\begin{array}{c}\text { Non-uniform } \\
\text { transmission line }\end{array}$ \\
\hline $\begin{array}{c}\text { Plane wave } \\
\text { (GTEM) }\end{array}$ & Case 1 & Case 2 \\
\hline $\begin{array}{c}\text { Random feld } \\
\text { (VIRC) }\end{array}$ & Case 3 & Case 4 \\
\hline
\end{tabular}


presented in [85], aims at comparing the four cases presented in Table 5.1 and analyzing the similarities and differences, possibly allowing for the creation of a simplified macro-parameter-based model usable as a quick "rule of the thumb" solution. The example macro-parameters are easy to obtain and include line length, distance between the connectors, and average height above the ground plane. Then, the error is considered when simplifications are made, i.e. approximating an unknown line with a uniform one.

\subsection{Measurement setups}

The measurement setup configurations are described in this section in two categories. The first one defines the different line geometries used in the experiment, and the second category is about the line excitation mechanisms.

\subsubsection{Line geometry}

The transmission line configurations are based on an improved test setup presented in [85]. All the lines have been built from a copper wire mounted above a $45 \mathrm{~cm} \times 35 \mathrm{~cm}$ ground plane, soldered to N-type connectors located $30 \mathrm{~cm}$ away from each other. Every line configuration begins and ends with a $4 \mathrm{~cm}$ riser, i.e. a wire perpendicular to the ground plane. In cases of the uniform transmission lines, the wire was always located $4 \mathrm{~cm}$ above the ground, therefore the total wire length was $38 \mathrm{~cm}$. For every case of non-uniform transmission lines, a $45 \mathrm{~cm}$ wire was used. To create different samples, the same wire was being randomly bent in the plane perpendicular to the ground plane, i.e. with variable height, but always averaging to $4 \mathrm{~cm}$. In total, a set of six non-uniform lines has been measured in the GTEM cell, and five lines in the reverberation chamber. Only the aforementioned macroparameters have been stored because, for the purpose of the experiment, the exact line geometries are assumed to be random, independent, and unknown. The photographs of example line configurations can be found in Table 5.2. The power was measured on one of the ports while the other one was being terminated with a short circuit.

\subsubsection{Line excitation}

As shown in Table 5.1, all the transmission lines were subjected to two different kinds of illumination: plane wave and random field, described below. 
Table 5.2: Example transmission line geometries.

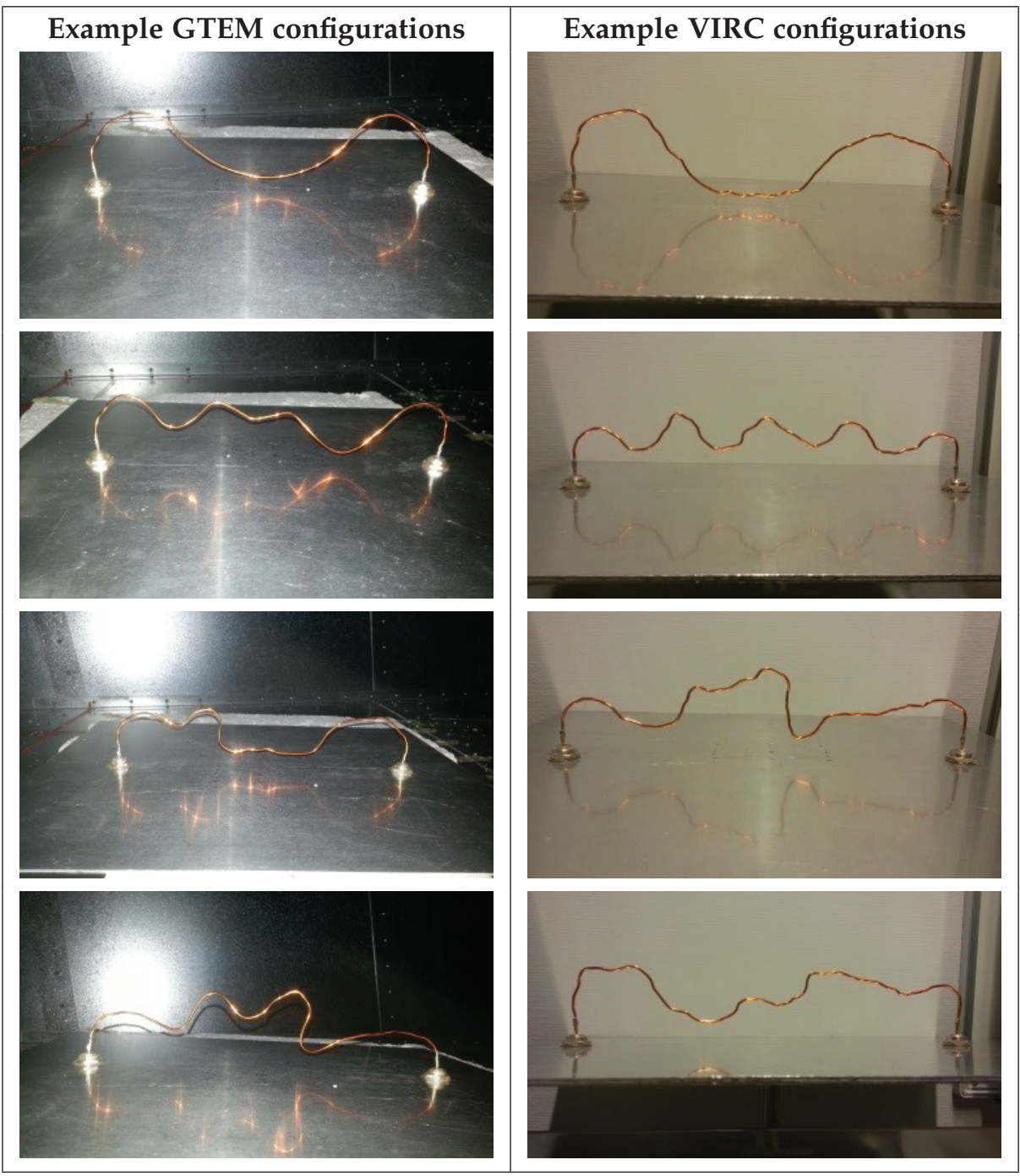




\section{Plane wave}

First, the illumination of the transmission lines by a plane wave was performed by placing them inside a GTEM cell. The measurements were performed in three different spatial orientations of the lines, causing broadside, sidefire, and endfire excitations, as defined in [76]. For each position, the $\left|S_{21}\right|$ parameters have been stored in the frequency range between $200 \mathrm{MHz}$ and $3 \mathrm{GHz}$. The selected frequency range covers the first resonance of the selected lines, as well as a region where the lines become electrically long. The used vector network analyzer had been calibrated to give results independent of the part of the setup other than the GTEM cell and the transmission line themselves. Assuming that the behavior of the GTEM cell does not change with frequency in this range, no further corrections have been made.

\section{Random field}

By subsequently placing the transmission lines inside the small VIRC, they were being illuminated by many different plane waves coming from different directions, with different amplitudes, phases, and polarizations. Due to the multipath nature of this environment, there is no need to place the transmission lines in more than a single spatial configuration. The $\left|S_{21}\right|$ parameters have been recorded over the frequency range between $200 \mathrm{MHz}$ and $3 \mathrm{GHz}$. The vector network analyzer had been calibrated between the transmitting antenna and the output port of the transmission line, therefore the results had to be compensated for the transmitting discone antenna mismatch. 500 samples have been obtained for each frequency point for each line.

\subsection{Experimental results}

The collective received power results for the non-uniform lines, calculated from the $\left|S_{21}\right|$ parameters with the output power set to $0 \mathrm{dBm}$, obtained for Case 1 and Case 2, i.e. plane wave illumination, are presented in Figures 5.1-5.3, corresponding to broadside, sidefire, and endfire excitations respectively. It can be easily observed that the changes in the geometry of the line have a significant impact on the dynamics of the received power, although there are certain similarities in the maximum levels of all the lines. The resonance frequencies vary only slightly among the non-uniform lines. Of course, the lines resonate differently for individual illumination angles due to very different coupling mechanisms. The average received power, calculated from the 500 sweeps in the VIRC, is presented in Figure 5.4. 


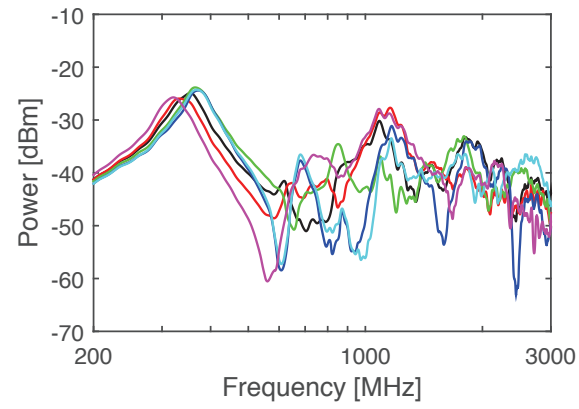

Figure 5.1: GTEM broadside

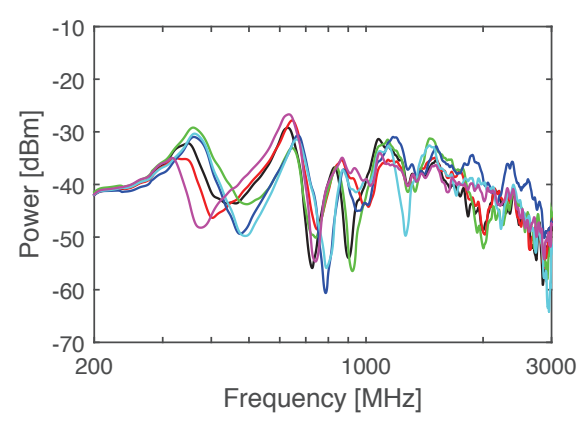

Figure 5.3: GTEM endfire illumination of the non-uniform lines.

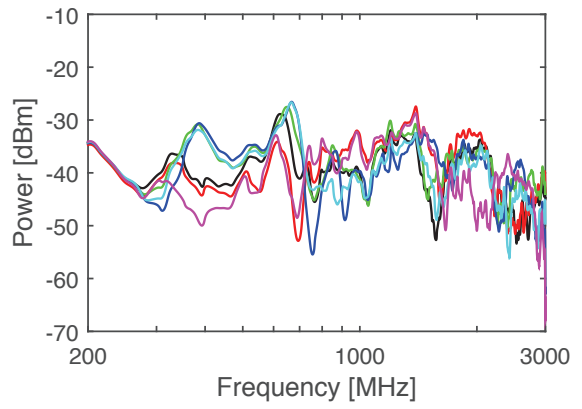

Figure 5.2: GTEM sidefire

illumination of the non-uniform lines. illumination of the non-uniform lines.

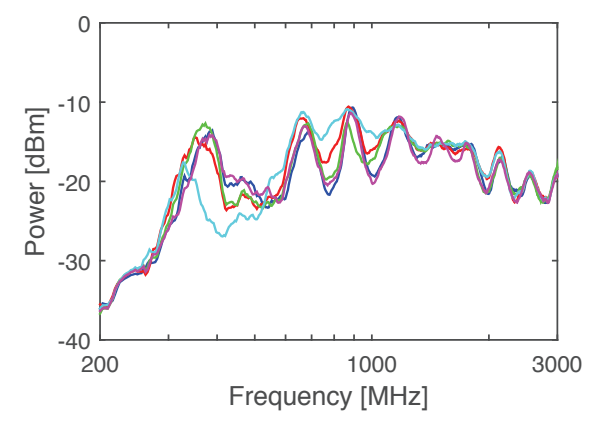

Figure 5.4: VIRC average results for the non-uniform lines.

Although the LUF of this chamber is around $300 \mathrm{MHz}$, the main focus is placed on the higher frequency range. In this figure, it can immediately be observed that the maximum level differences between the lines are rather low. Furthermore, although repeated resonance peaks are present, the overall frequency response of the lines is more flat than in previous cases due to overlapping resonances in the multipath environment (all of the coupling mechanisms are active, including broadside, sidefire, and endire). The repeatability of the results is expressed using the standard deviation $(\sigma)$ of the powers received by the non-uniform lines, shown in Figure 5.5. It can be observed that the $\sigma$ of the lines illuminated from the three directions independently in the GTEM cell (Case 2) is significantly higher than the $\sigma$ of the lines measured in the VIRC (Case 4). Interestingly, while the $\sigma$ in Case 2 becomes larger with frequency due to the amount of different resonances, the opposite effect can be observed in Case 4. In the latter case, all of the measured transmission lines begin to act in a very similar manner, resulting in a decrease in the standard deviation, implying that the line geometry becomes gradually irrelevant. 
Since the measurements conducted in the VIRC are expected to give the worst case result, i.e. highest coupling, they have additionally been compared with the total field coupling result obtained in the GTEM cell, calculated using the 3-point vectorial sum obtained by the means of broadside, sidefire, and endfire excitations, as described in [89] and [90], for each frequency point. Although this result should be comparable to the VIRC result, it is not, meaning that more measurement angles should be taken in the GTEM to obtain the results with a similarly high accuracy to the VIRC. Similar $\sigma$ results have been obtained when the lines were terminated with a $50 \mathrm{Ohm}$ load, shown in Figure 5.6.

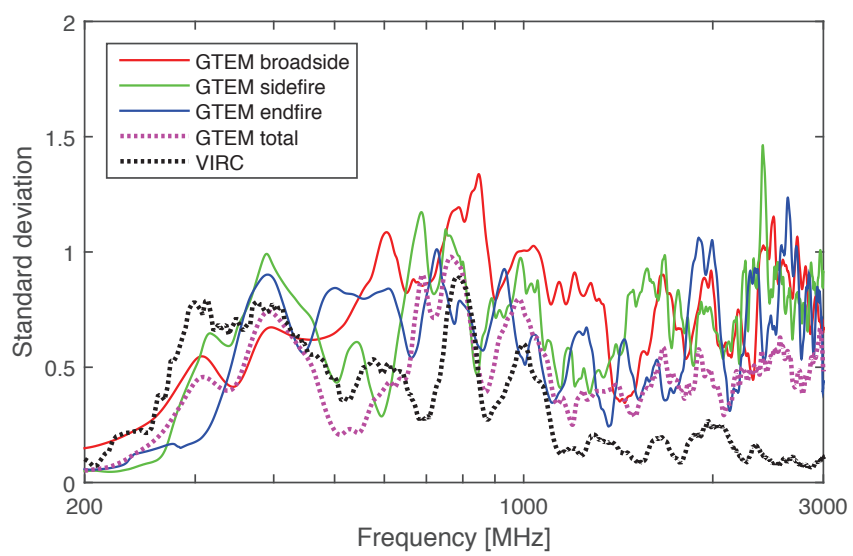

Figure 5.5: Standard deviations for short line terminations.

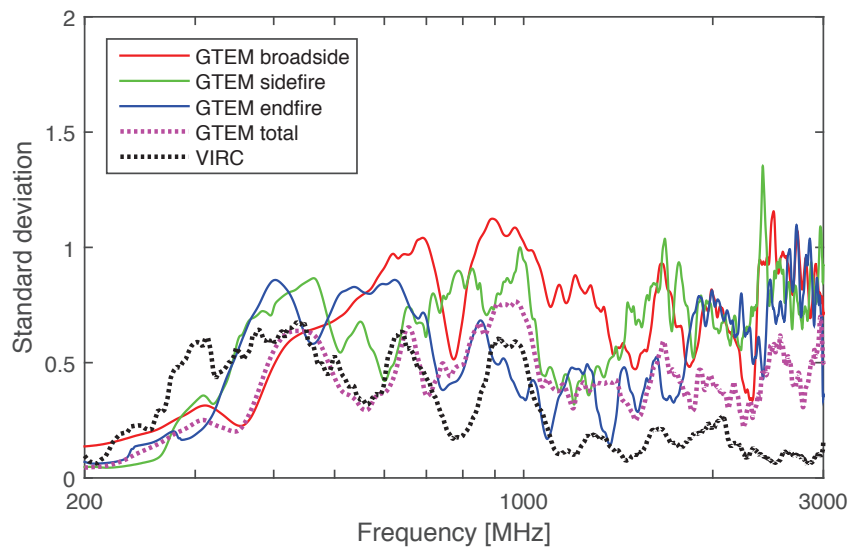

Figure 5.6: Standard deviations for 50 Ohm line terminations. 


\subsection{Simplifications towards macro-parameters for unstructured transmission lines}

A simple macro-parameter-based analysis is more desirable than "accurate but unpredictable" solutions from the EMC's point of view. This unpredictable feeling exists in industry where after weeks of simulation the worst case situation cannot be determined using numerical tools, because the exact configuration of wiring and fields has to be known. Following the observation regarding the converging results in Case 4 from the previous section, i.e. the similarities between coupling to non-uniform lines placed in random fields, this section focuses on analyzing the error when a strong simplification further affecting the line geometry is made. It is desired to create a macro-parameter model predicting the absolute coupling levels to non-uniform transmission lines, with incorporated expected distributions of parameters due to line geometries, as in [85]. The initial, practical step, is to use the simplest possible solution, a uniform line. This model, as a subject of Case 1 and Case 3, has been thoroughly studied and is most attractive for both analytical and numerical analysis. Similarly to the approach adopted in the previous chapters, the deviations from the perfect (uniform line) case, caused by the imperfections of reality, are presented here.

The measured power coupled into the uniform line are plotted on top of the maximum and average powers received by the non-uniform lines in Figures 5.7 - 5.10, for the GTEM broadside, sidefire, endfire, and the VIRC, respectively.

For each frequency point, the results follow a certain distribution caused by the variations of the line geometry, e.g. Gamma, as suggested in [85]. Although only a very limited amount of different line geometries was measured here, the obtained difference between the maximum and average power is consistent in the GTEM cases. Such a consistency could possibly give some space for a max/avg model of predicting the maximum coupling, similar to the one used in Chapter 3. However, this is not the case in the multipath environment, where the two traces converge in the higher frequency range, as already indicated by the $\sigma$ analyzed in the previous section. The uniform line measurements performed in the GTEM (Case 1), plotted in the same figures, provide highly different results compared to the non-uniform lines (Case 2). These differences between the maximum and average non-uniform lines and the uniform line are shown in Figure 5.11 and 5.12, respectively. The difference is highest for the broadside excitation, up to $30 \mathrm{~dB}$, and up to $10-20 \mathrm{~dB}$ for sidefire and endfire excitations. The results are about 2-3 dB smaller when comparing the uniform line to the average power of the non-uniform lines. However, the error associated with such a strong simplification, obtained in the multipath environment of the VIRC, is significantly smaller, consistently 


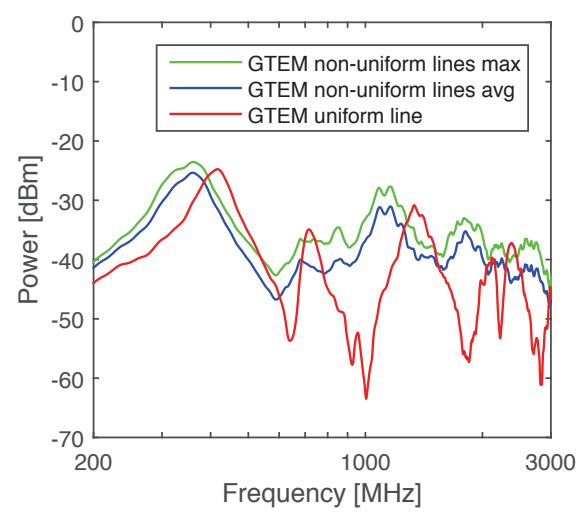

Figure 5.7: GTEM broadside excitation, short termination.

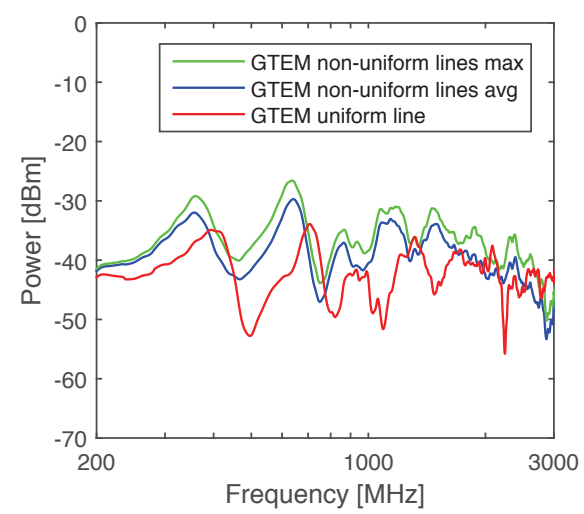

Figure 5.9: GTEM endfire excitation, short termination.

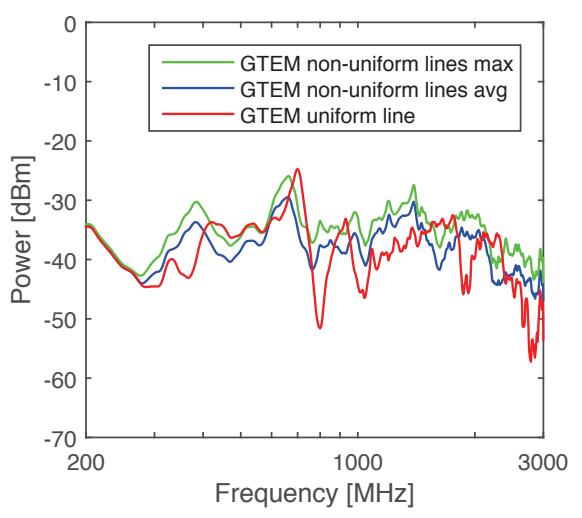

Figure 5.8: GTEM sidefire excitation, short termination.

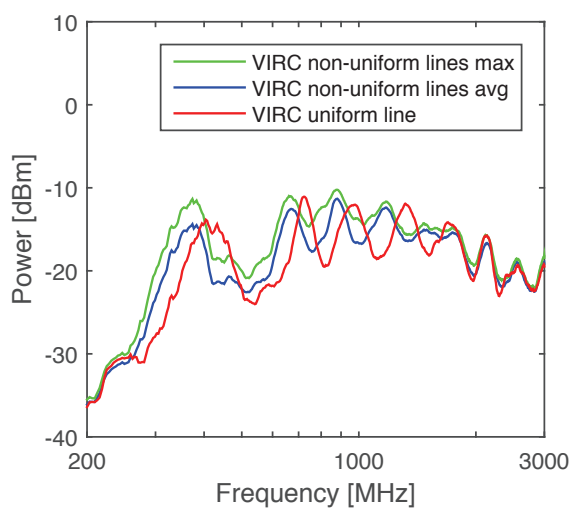

Figure 5.10: VIRC average results, short termination.

below $10 \mathrm{~dB}$, and approaching $0 \mathrm{~dB}$ with increased frequency. Although slightly lower when calculated with respect to the average power, the peaks shown in Figure 5.10 are on a level close to the peaks of the maximums of the non-uniform lines. The apparent frequency shift below $2 \mathrm{GHz}$ is caused by the differences in the line lengths. If the measured lines were designed with a constant length rather than distance from the ports, the results would overlap better. Then, it would be possible to predict the risk of maximum coupling to non-uniform lines using a uniform one with only a very small error. On the other hand, the maximum coupling to the random lines does not exhibit as strong resonant behavior as in case of the uniform line, i.e. it is more flat. Considering the results from [85], it can be assumed that the 
predicted maximum levels do not inherit the resonant behavior, therefore a decent estimation could be obtained by connecting the peaks of the uniform line above $700 \mathrm{MHz}$. However, due to a very strong convergence of the data above $2 \mathrm{GHz}$, it can be safely stated that the line geometry becomes irrelevant due to the highly overlapping line resonances. The results obtained for a 50 Ohm line termination, shown in Figures 5.13 and 5.14, indicate an even better fit.

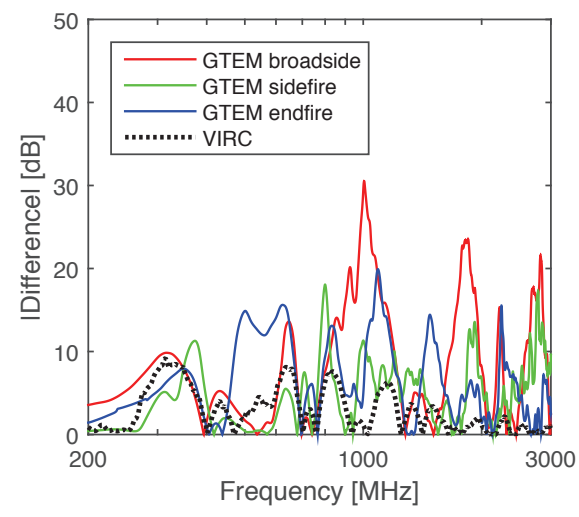

Figure 5.11: Difference between the uniform line and the maximum of the non-uniform lines, short termination.

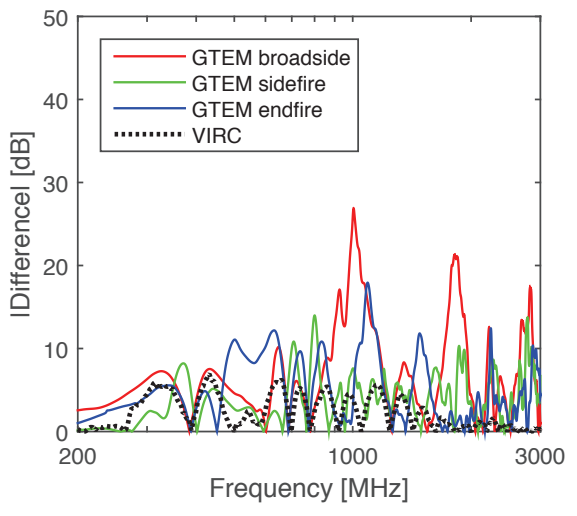

Figure 5.12: Difference between the uniform line and the average of the non-uniform lines, short termination.

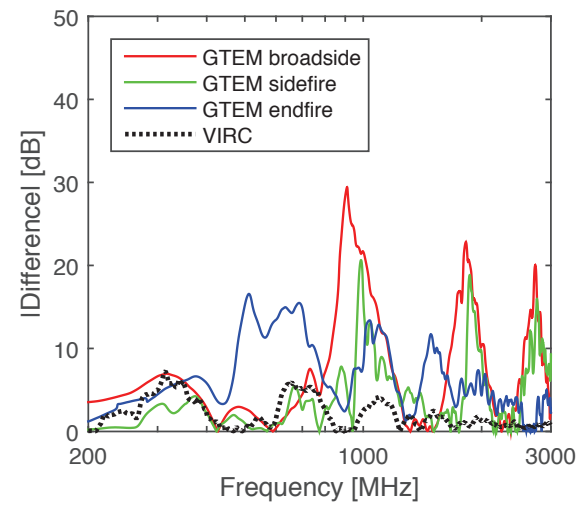

Figure 5.13: Difference between the uniform line and the maximum of the non-uniform lines, $50 \mathrm{Ohm}$ termination.

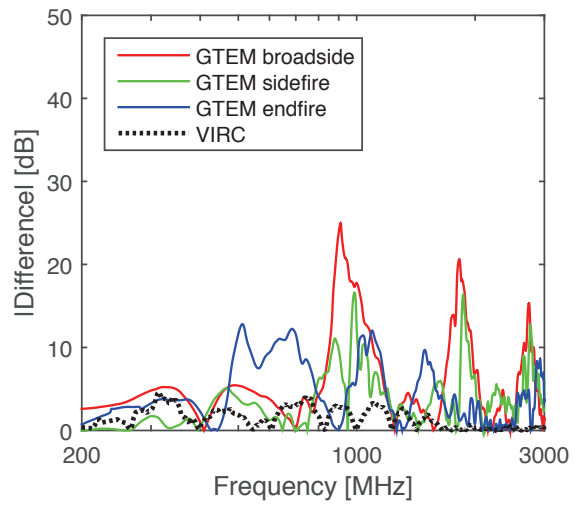

Figure 5.14: Difference between the uniform line and the average of the non-uniform lines, $50 \mathrm{Ohm}$ termination. 


\subsection{Summary and discussion}

In this chapter, 4 cases of field coupling into transmission lines have been experimentally analyzed. A small set of randomly bent, non-uniform lines has been measured in a GTEM cell and in the VIRC. All the built transmission lines are described using only basic macro-parameters: line length, distance between the connectors, and average height above the ground plane. The analysis is mainly addressed at finding the differences between the given configurations, and exposing the simplifications caused by the randomness of both the line geometry and the coupled field. The analysis of a further simplification, using a uniform line instead of non-uniform ones, is aimed at finding the maximum threat by using only easy to model and well established solutions.

From the measurements performed in the GTEM cell, it is concluded that a significant spread of results can be expected depending on the wire geometry and excitation direction, leading to large variations in both resonance frequencies and received power levels. To avoid the difficulties of modeling a geometrically complex line, as well as eliminating the high sensitivity to the assumed parameters, a statistical approach utilizing the multipath response is preferred.

The total coupling can be calculated by performing a set of three measurements for each line but the process is quite time-consuming and does not even provide the results with the accuracy of the VIRC. In the latter case, total (multipath) coupling can be obtained in a much faster and simpler way, as only one spatial position is required.

In real REs, both the line and the excitation are random. Instead of multiplying the complexities of analytical or numerical models, a simplified model can be created. It has been shown that in the multipath environment, the differences between the non-uniform lines with the same macro-parameters become smaller, especially in the higher frequency range. The coupling of the overmoded field from various directions causes many overlapping resonances on the line. From the experimental analysis presented here, the results indicate differences between o and $10 \mathrm{~dB}$ when simplifying the complex geometrical structures with the uniform line, especially when comparing lines of similar lengths rather than distances from the ports. 


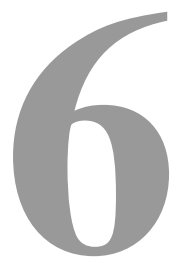

\section{Conclusions and future work}

In deterministic EM models, where with increasingly specified parameters the result is more precise, its accuracy depends largely on the quality of the input data. In the area of statistical EM, many parameters are assumed to be unknown, which gives a certain degree of freedom. This freedom allows to bypass the unreasonable or even currently impossible tasks, such as e.g. exact modeling of a reverberant environment, broadening the spectrum of applicable solutions. The resulting simplification is the key to making complex things usable. However, "everything should be made as simple as possible, but not simpler". Therefore, it is of crucial importance to find and evaluate the link between the established models and the explored possibilities. This approach has been adopted throughout this work utilizing experimental measurements to validate, extend, or simplify the existing models, providing suggestions for usable solutions, as well as a fresh view on the subject.

\section{Chapter 2}

In Chapter 2, the definition of REs has been introduced as environments unintentionally possessing certain capabilities to create resonating fields, resembling those inside a dedicated RC. Affected by the significant imperfections with respect to the laboratory facilities, such as poor shielding, lossy objects, non-uniform distribution of reflectors and apertures, the field statistics can vary significantly. However, depending on the conditions in a given RE, they 
can be successfully described using the RC models, inheriting the practically useful features allowing to predict the distributions with high repeatability, as well as create potential EMI threats in the resonant field.

The experimental analysis has been performed on two examples of REs: a typical office environment and an industrial workshop, additionally referred to a classical RC. The obtained Q-factor and IL values, as well as the results of a GOF test were compared, indicating a large spread of results, also with a strong frequency dependency. It has been concluded that the analyzed REs, especially the workshop, can act similarly to the RC. The results obtained in the lower Q-factor office expressed a stronger deviation from the theoretical model, although still possessing obvious reverberant properties. Furthermore, due to the difficulty of collecting large amounts of data and parameter isolation in the REs, two chambers have been analyzed as candidates allowing to simulate an RE in laboratory conditions. By the means of Q-factor, k-factor, GOF, as well as number of samples, related to field repeatability, it has been shown that the VIRC provides substantially better conditions than a classical RC for the given purpose.

Because only two REs were analyzed in this work, it would be desirable to perform additional measurements in different environments, possibly collecting more data. A larger measurement campaign could allow to point out the critical parameters differentiating the REs, as well as define a specified threshold when RC techniques can be applied.

\section{Chapter 3}

Following the results obtained in Chapter 2, Chapter 3 focused on exploiting the unique capabilities of the VIRC to deliver large amounts of uncorrelated data, in search of the maximum realistically obtainable field strengths in reverberant environments. Such a knowledge would allow to ensure the prevention of possible EMI problems. The analysis of the local maximum occurrence in the oversampled data indicated that the initial 1-hour long measurements are reasonable, showing that new field configurations creating those maximums appear within such a long time, and with high repeatability. In order to modify the existing max/avg models, and also validate them for use in imperfect REs, the measurements were performed in variable conditions. Altering the Q-factor from around 125 in a heavily loaded small VIRC up to 4000 in the big VIRC, all with very different mode densities, did not allow to successfully prove the stated hypothesis that the max/avg ratio is a function of the Q-factor. The results obtained in those cases showed a consistent value of around 12-13 $\mathrm{dB}$ max/avg electric field ratio with no effect due to the altered conditions. 
Because a single measurement result obtained in the empty VIRC indicated a skew in the PDF, an investigation aimed at understanding the origin of this artifact led to another series of measurements with variable receiving monopole antenna dimensions. The measurements were performed using two entirely different setups and techniques, but leading to the same results. A strong dependency of the max/avg ratio on the receiving antenna has been observed, giving almost $20 \mathrm{~dB}$ max/avg for short, and $14 \mathrm{~dB}$ for long monopoles. On the other hand, a resonant monopole lowered the ratio down to $10 \mathrm{~dB}$. It was found out that such a drastic difference in the results is related to the skew of the distribution when operating outside of the resonance, leaning towards a Weibull distribution. Furthermore, loading the chamber with an absorber shifts the distribution back towards Rayleigh, although affecting the max/avg ratio mostly for antennas below the resonant length.

Although the measurements were performed twice with overlapping conclusions, it is recommended to validate the initial observations by the means of more extensive measurements or numerical simulations, as well as formulation of an analytical expression describing the observed effect. The highly variable max/avg ratio and its dependency on the antenna length is of a very high importance for both RC measurements, where maximum values are commonly used, as well as estimating the risk of EMI in REs. Additionally, the effects of the transmitting antenna properties should be addressed in the same way.

\section{Chapter 4}

The analysis of alternative methods of measuring radiated emissions on-site has been performed in Chapter 4. Because of the better fit to the scope of this thesis, a stronger focus, including experimental evaluation, was placed on the STS as a solution applicable in REs. The measurements were performed in the two environments, previously analyzed in Chapter 2 (an office and a workshop), utilizing the obtained IL data for the purpose of both calibrating the sites to get the TRP results, as well as calculating the uncertainty levels. The actual radiated emission measurements were performed using a comb generator to simulate an immovable apparatus, and a portable SA as the receiver. The measurement was based on the IEC 61000-4-21 standard and the utilizing the volume sampling technique. Although not as accurate as laboratory tests, it has been shown that it is possible to perform on-site measurements with only an unexpectedly low error of $5 \mathrm{~dB}$ to $10 \mathrm{~dB}$ in the performed experiments. Corrections for the receiver nonlinearity when measuring low SNR signals are necessary. The discussed method is fast and allows to perform emission measurements on a site even with a very limited space for line of sight measurements, without the necessity to perform much 
more precise scanning around the apparatus. Although using maximum readings increases the measurement uncertainty due to lower repeatability, it provides higher SNR, thus sensitivity, by around 10 dB. On the other hand, using the more repeatable average values leads to the risk of losing the signal in the noise floor, especially malicious in the unshielded REs where other equipment is present.

Although the presented results are very promising, the presented STS method is only usable for measuring strong emissions in quiet environments because only the highly attenuated scattered components are received. Otherwise, the suggested ATS method could be of use, due to the utilization of the strong LOS component.

\section{Chapter 5}

Four cases, plane wave or random field coupled to uniform or non-uniform transmission lines, have been discussed in Chapter 5. An experimental analysis of a small set of non-uniform lines, randomly bent, exposed to plane wave as well as random field illumination was performed in a GTEM cell and the VIRC, respectively. The search of any similarities and possible simplifications was the chosen method of creating a simplified model based only on basic macroparameters: line length, distance between the connectors, and average height above the ground plane. The model could be used to predict the EMI threat in cases where the exact line geometry is unknown, and the environment is undefined, therefore assumed to be (semi)reverberant. The randomness of the line structure combined with the randomness of the coupled field allows to neglect the details, normally critical in analytical calculations. It has been shown that all the non-uniform lines behave similarly, especially at higher frequencies, where the line geometry becomes irrelevant. Then, a further simplification was introduced - substituting the non-uniform lines with a uniform one, in order to predict the maximum coupling. From the experimental analysis presented, the results indicated no major differences when simplifying the complex geometrical structures with the uniform line, especially when comparing lines of similar lengths rather than distances from ports.

Although the presented experimental work was limited to only a couple of samples, a decent base for future research has been established. Future work should include an increased number of samples allowing a deeper statistical analysis, along with a greater variation of the macro parameters and the analysis of their impact on the final outcome. 


\section{Bibliography}

[1] H. Mendes, "A new approach to electromagnetic field strength measurements in shielded enclosures", in Wescon Technical Papers, Western Electronic Show and Convention, Los Angeles, 1968.

[2] Reverberation chamber test methods. IEC 61000-4-21, 2011.

[3] HIRF-SE project. [Online]. Available: http://hirf se.axessim.eu/.

[4] PEM project. [Online]. Available: http://cordis . europa.eu/project/ $\mathrm{rcn} / 85118$ en.html.

[5] EMRP project. [Online]. Available: https://www. emc-industry . com/.

[6] R. E. Richardson, "Reverberant microwave propagation", Dahlgren Division Naval Surface Warfare Center, Virginia, Tech. Rep. NSWCDD/TR08/127, 2008.

[7] A. Pomianek, K. Staniec, and Z. Jóskiewicz, "Practical remarks on measurement and simulation methods to emulate the wireless channel in the reverberation chamber", Progress in Electromagnetics Research-pier PROG ELECTROMAGN RES., vol. 105, pp. 49-69, 2010.

[8] A. F. Molish, Wireless Communications. John Wiley \& Sons, Inc., 2011.

[9] J. G. Kostas and B. Boverie, "Statistical model for a mode-stirred chamber", IEEE Transactions on Electromagnetic Compatibility, vol. 33, no. 4, 1991.

[10] D. M. Pozar, Microwave Engineering, 3rd Edit. John Wiley \& Sons, Inc., 2005.

[11] A. Cozza, "The role of losses in the definition of the overmoded condition for reverberation chambers and their statistics", IEEE Transactions on Electromagnetic Compatibility, vol. 53, no. 2, 2011.

[12] R. Serra, "Introduction of randomness in deterministic, physicallyconsistent descriptions of reverberation chambers and experimental verification", PhD thesis, Politecnico di Torino, Turin, Italy, 2009. 
[13] S. van de Beek, K. A. Remley, C. L. Holloway, J. M. Ladbury, and F. Leferink, "Characterizing large-form-factor devices in a reverberation chamber", in International Symposium on Electromagnetic Compatibility, EMC EUROPE 2013, Brugge, 2013.

[14] R. Vogt-Ardatjew, S. van de Beek, and F. Leferink, "Wide-band antennas for reverberation chambers", in International Symposium on Electromagnetic Compatibility, EMC EUROPE 2012, Rome, 2012.

[15] F. Leferink, J.-C. Boudenot, and W. van Etten, “Experimental results obtained in the vibrating intrinsic reverberation chamber", in IEEE International Symposium on Electromagnetic Compatibility, Washington, 2000.

[16] A. Ubin, R. Vogt-Ardatjew, F. Leferink, M. Zarar, M. Jenu, and S. van de Beek, "Statistical analysis of three different stirrer designs in a reverberation chamber", in Asia-Pacific Symposium on Electromagnetic Compatibility, APEMC 2015, Taipei, 2015.

[17] R. Serra and F. Leferink, "Statistical measurements of fast changing electromagnetic fields", in International Symposium on Electromagnetic Compatibility, EMC EUROPE 2010, Wrocław, 2010.

[18] D. Hill, Electromagnetic Fields in Cavities. John Wiley \& Sons, Inc., 2009.

[19] E. Genender, C. L. Holloway, K. A. Remley, J. M. Ladbury, and G. Koepke, "Simulating the multipath channel with a reverberation chamber: application to bit error rate measurements", IEEE Transactions on Electromagnetic Compatibility, vol. 52, no. 4, 2010.

[20] H. G. Krauthauser, "On the measurement of total radiated power in uncalibrated reverberation chambers", IEEE Transactions on Electromagnetic Compatibility, vol. 49, no. 2, 2007.

[21] L. R. Arnaut and G. Gradoni, "Probability distribution of the quality factor of a mode-stirred reverberation chamber", IEEE Transactions on Electromagnetic Compatibility, vol. 55, no. 1, 2013.

[22] C. L. Holloway, D. A. Hill, J. M. Ladbury, P. F. Wilson, G. Koepke, and J. Coder, "On the use of reverberation chambers to simulate a rician radio environment for the testing of wireless devices", IEEE Transactions on Antennas and Propagation, vol. 54, no. 11, 2006.

[23] D. Hill, "Spatial correlation function in RC", IEEE Transactions on Electromagnetic Compatibility, vol. 37, no. 1, 1995.

[24] F. Monsef, R. Serra, and A. Cozza, "Goodness-of-fit tests in reverberation chambers: is sample independence necessary?", IEEE Transactions on Electromagnetic Compatibility, vol. 57, no. 6, 2015. 
[25] P. Hallbjörner, "A model for the number of independent samples in reverberation chambers", Microwave and Optical Technology Letters, vol. 33, no. 1, 2002.

[26] - "Estimating the number of independent samples in reverberation chamber measurements from sample differences", IEEE Transactions on Electromagnetic Compatibility, vol. 48, no. 2, 2006.

[27] C. Lemoine, P. Besnier, and M. Drissi, "Estimating the effective sample size to select independent measurements in a reverberation chamber", IEEE Transactions on Electromagnetic Compatibility, vol. 50, no. 2, 2008.

[28] S. Pfennig, "A general method for determining the independent stirrer positions in reverberation chambers: adjusting the correlation threshold", IEEE Transactions on Electromagnetic Compatibility, vol. 58, no. 4, 2016.

[29] C. Lemoine, P. Besnier, and M. Drissi, "Investigation of reverberation chamber measurements through high-power goodness-of-fit tests", IEEE Transactions on Electromagnetic Compatibility, vol. 49, no. 4, 2007.

[30] V. M. Primiani and F. Moglie, "Numerical simulation of reverberation chamber parameters affecting the received power statistics", IEEE Transactions on Electromagnetic Compatibility, vol. 54, no. 3, 2012.

[31] R. Vogt-Ardatjew, S. van de Beek, and F. Leferink, “Experimental extreme field strength investigation in reverberant enclosures", in International Symposium on Electromagnetic Compatibility, EMC EUROPE 2014, Gothenburg, 2014.

[32] F. Monsef and A. Cozza, "Average number of significant modes excited in a mode-stirred reverberation chamber", IEEE Transactions on Electromagnetic Compatibility, vol. 56, no. 2, 2014.

[33] Emission Standard for Industrial Environments. IEC 61000-6-4, 2006.

[34] C. L. Holloway, D. A. Hill, J. M. Ladbury, and G. Koepke, "Requirements for an effective reverberation chamber: unloaded or loaded", IEEE Transactions on Electromagnetic Compatibility, vol. 48, no. 1, 2006.

[35] D. Hill, "Plane wave integral representation for fields in reverberation chambers", IEEE Transactions on Electromagnetic Compatibility, vol. 40, no. 3, 1998.

[36] T. Lehmann and G. Freyer, "Characterization of the maximum test level in a reverberation chamber", in IEEE International Symposium on Electromagnetic Compatibility, Austin, 1997.

[37] J. Ladbury, G. Koepke, and D. Camell, "Evaluation of the NASA Langley mode-stirred facility”, NIST, Tech. Rep. 1508, 1999.

[38] L. R. Arnaut, "Measurement uncertainty in reverberation chambers sample statistics", NPL, Tech. Rep. TQE 2, 2008. 
[39] Z. Daming, S. K. Yak, and K. W. Jin, "Prediction of maximum electric field with given independent sampling points in a reverberation chamber", in 17th International Zurich Symposium on Electromagnetic Compatibility, Zurich, 2006.

[40] M. Höijer, "Maximum power available to stress onto the critical component in the equipment under test when performing a radiated susceptibility test in the reverberation chamber", IEEE Transactions on Electromagnetic Compatibility, vol. 48, no. 2, 2006.

[41] - "Maximum levels inside an electromagnetic shielded cavity", in IEEE International Symposium on Electromagnetic Compatibility, Fort Lauderdale, 2010.

[42] M. Höijer, L. Kroon, and O. Lundén, "A tool to calculate the worst electromagnetic threat in cavities", in International Symposium on Electromagnetic Compatibility, EMC EUROPE 2012, Rome, 2012.

[43] A. Gifuni, "Deterministic approach to estimate the upper bound of the electric field in a reverberation chamber", IEEE Transactions on Electromagnetic Compatibility, vol. 53, no. 3, 2011.

[44] R. Serra, "Evanescent reverberation", in International Symposium on Electromagnetic Compatibility, EMC EUROPE 2012, Rome, 2012.

[45] R. Serra and F. G. Canavero, "Bivariate statistical approach for good-butimperfect electromagnetic reverberation", IEEE Transactions on Electromagnetic Compatibility, vol. 53, no. 3, 2011.

[46] G. Orjubin, E. Richalot, S. Mengué, and O. Picon, "Statistical model of an undermoded reverberation chamber", IEEE Transactions on Electromagnetic Compatibility, vol. 48, no. 1, 2006.

[47] R. A. Fisher and L. H. C. Tippett, "Limiting forms of the frequency distribution of the largest or smallest member of a sample", Mathematical Proceedings of the Cambridge Philosophical Society, vol. 24, no. 02, 1928.

[48] G. Orjubin, "Maximum field inside a reverberation chamber modeled by the generalized extreme value distribution", IEEE Transactions on Electromagnetic Compatibility, vol. 49, no. 1, 2007.

[49] C. E. Hager, J. D. Rison, and G. B. Tait, "Electromagnetic probabilityof-effect assessment tool for high-power susceptibility testing", IEEE Transactions on Electromagnetic Compatibility, vol. 58, no. 4, 2016.

[50] N. Wellander, O. Lunden, and M. Backstrom, "The maximum value distribution in a reverberation chamber", in IEEE International Symposium on Electromagnetic Compatibility, Montreal, 2001.

[51] F. Leferink, "Maximum and average field strength in enclosed environments", in International Symposium on Electromagnetic Compatibility, EMC EUROPE 2010, Wrocław, 2010. 
[52] —, "Fast, broadband, and high-dynamic range 3-D field strength probe", IEEE Transactions on Electromagnetic Compatibility, vol. 55, no. 6, 2013.

[53] R. Vogt-Ardatjew, R. Serra, L. G. Hiltz, and F. Leferink, "Response time of electromagnetic field strength probes", in Asia-Pacific Symposium on Electromagnetic Compatibility, APEMC 2013, Melbourne, 2013.

[54] R. Vogt-Ardatjew and F. Leferink, "Observation of maximal and average field values in a reverberation chamber", in International Symposium on Electromagnetic Compatibility, EMC EUROPE 2013, Brugge, 2013.

[55] R. Vogt-Ardatjew, S. van de Beek, and F. Leferink, "Influence of reverberation chamber loading on extreme field strength", in International Symposium on Electromagnetic Compatibility, Tokyo, 2014.

[56] D. A. Hill, "A reflection coefficient derivation for the $Q$ of a reverberation chamber", IEEE Transactions on Electromagnetic Compatibility, vol. 38, no. 4, 1996.

[57] B. H. Liu, D. C. Chang, and M. T. Ma, "Eigenmodes and the composite quality factor of a reverberating chamber", NBS, Tech. Rep. 1066, 1983.

[58] S. Pfennig and H. G. Krauthäuser, "Comparison of methods for determining the number of independent stirrer positions in reverberation chambers", in International Symposium on Electromagnetic Compatibility, EMC EUROPE 2013, Brugge, 2013.

[59] J. B. Gros, U. Kuhl, O. Legrand, and F. Mortessagne, "Lossy chaotic electromagnetic reverberation chambers: universal statistical behavior of the vectorial field", Physical Review E - Statistical, Nonlinear, and Soft Matter Physics, vol. 93, no. 3, 2016.

[6o] C. Lemoine, P. Besnier, and M. Drissi, "Effect of the size of the antenna on measurements distribution in reverberation chamber", in 18th International Zurich Symposium on Electromagnetic Compatibility, Zurich, 2007.

[61] H. Siasi, "Validation of the vibrating intrinsic reverberation chamber", Thales, Hengelo, Tech. Rep., 2001.

[62] J. F. Dawson, T. Konefal, M. P. Robinson, A. C. Marvin, S. J. Porter, and L. C. Chirwa, "Field statistics in an enclosure with an aperture effect of Q-factor and number of modes", in International Symposium on Electromagnetic Compatibility, EMC 2005, Chicago, 2005.

[63] R. Vogt-Ardatjew, A. E. Sowa, and F. Leferink, "The effect of magnetodielectric absorbing coating on unsymmetrical antenna cables", in AsiaPacific Symposium on Electromagnetic Compatibility, APEMC 2015, Taipei, 2015. 
[64] J. M. Ladbury and D. A. Hill, "Enhanced backscatter in a reverberation chamber: inside every complex problem is a simple solution struggling to get out", in IEEE International Symposium on Electromagnetic Compatibility, EMC 2007, Zurich, 2007.

[65] F. Leferink, "Optimal utilization of a reverberation chamber", in 4th European Symposium on Electromagnetic Compatibility, Brugge, 2000.

[66] G. B. Tait and M. B. Slocum, "Random-walk technique for measuring the electromagnetic environment in electrically large reflective spaces", IEEE Transactions on Instrumentation and Measurement, vol. 60, no. 3, 2011.

[67] G. B. Tait, C. E. Hager IV, T. T. Baseler, and M. B. Slocum, “Ambient power density and electric field from broadband wireless emissions in a reverberant space", IEEE Transactions on Electromagnetic Compatibility, vol. 58, no. 1, 2016.

[68] Radiated, radio-frequency, electromagnetic field immunity test. IEC 61000-4-3, 2006.

[69] Y. Lustmann and D. Porrat, "Indoor channel spectral statistics, k-factor and reverberation distance", IEEE Transactions on Antennas and Propagation, vol. 58, no. 11, 2010.

[70] B. Menssen, H. Brech, and H. Garbe, "Measurement validation for the extension of emission measurements in alternative test sites above 1 $\mathrm{GHz}^{\prime \prime}$, IEEE Transactions on Electromagnetic Compatibility, vol. 58, no. 4, 2016.

[71] P. F. Wilson, D. A. Hill, and C. L. Holloway, "On determining the maximum emissions from electrically large sources", IEEE Transactions on Electromagnetic Compatibility, vol. 44, no. 1, 2002.

[72] T. P. Ryan, Modern Engineering Statistics. John Wiley \& Sons, Inc., 2007.

[73] F. Leferink, D. J. G. Boerle, and F. A. G. Sogtoen, "In-situ EMI measurements using a vibrating intrinsic reverberation chamber", in 14th International Symposium and Technical Exhibition on Electromagnetic Compatibility, Zurich, 2001.

[74] C. R. Paul, Introduction to Electromagnetic Compatibility, 2nd Edit. John Wiley \& Sons, Inc., 2006.

[75] A. K. Agrawal, H. J. Price, and S. H. Gurbaxani, “Transient response of multiconductor transimssion lines excited by a nonuniform electromagetic field", IEEE Transactions on Electromagnetic Compatibility, vol. 22, no. 2, 1980 .

[76] C. Paul, Analysis of multiconductor transmission lines. John Wiley \& Sons, Inc., 1994 . 
[77] F. Rachidi, "A review of field-to-transmission line coupling models with special emphasis to lightning-induced voltages on overhead lines", IEEE Transactions on Electromagnetic Compatibility, vol. 54, no. 4, 2012.

[78] M. Ridel and J. P. Parmantier, "Determination of EM coupling on an electrical wiring interconnection system - application of condensation approaches on cable models", in International Symposium on Electromagnetic Compatibility, Tokyo, 2014.

[79] M. Magdowski and R. Vick, "Closed-form formulas for the stochastic electromagnetic field coupling to a transmission line with arbitrary loads", IEEE Transactions on Electromagnetic Compatibility, vol. 54, no. 5, 2012.

[8o] M. Magdowski, S. V. Tkachenko, and R. Vick, "Coupling of stochastic electromagnetic fields to a transmission line in a reverberation chamber", IEEE Transactions on Electromagnetic Compatibility, vol. 53, no. 2, 2011.

[81] H. Zhang, X. Zhao, L. Yan, C. Liu, and K. Huang, "An alternative semianalytical/analytical solution to field-to-wire coupling in an electrically large cavity", IEEE Transactions on Electromagnetic Compatibility, vol. 54, no. 1, 2012.

[82] D. Pissoort, F. Vanhee, B. Boesman, J. Catrysse, G. Vandenbosch, and G. Gielen, "High-frequency coupling to cables for plane wave and random wave conditions", in ESA Workshop on Aerospace EMC, Venice, 2012.

[83] I. Junqua, J.-P. Parmantier, and P. Degauque, "Field-to-wire coupling in an electrically large cavity: a semianalytic solution", IEEE Transactions on Electromagnetic Compatibility, vol. 52, no. 4, 2010.

[84] P. Manfredi and F. G. Canavero, "Polynomial chaos for random field coupling to transmission lines", IEEE Transactions on Electromagnetic Compatibility, vol. 54, no. 3, 2012.

[85] F. Diouf, F. Canavero, F. Leferink, and F. Buesink, "Field coupling to randomly-routed wires: theoretical and experimental comparison", in International Symposium on Electromagnetic Compatibility, EMC EUROPE 2010, Wrocław, 2010.

[86] O. O. Sy, J. A. H. M. Vaessen, M. C. van Beurden, B. L. Michielsen, A. G. Tijhuis, A. P. M. Zwamborn, and J. Groot, "Experimental validation of the stochastic model of a randomly fluctuating transmission-line", in 38th European Microwave Conference, EuMC 2008, Amsterdam, 2008.

[87] O. O. Sy, M. C. van Beurden, and B. L. Michielsen, "Analysis of stochastic resonances in electromagnetic couplings to transmission lines", in 2oth International Zurich Symposium on Electromagnetic Compatibility, Zurich, 2009. 
[88] A. Cozza and F. Canavero, "High-frequency extension of the transmissionline theory for an open line", in CEMo4, Toulouse, 2004.

[89] T. E. Harrington, “Total-radiated-power-based OATS-equivalent emissions testing in reverberation chambers and GTEM cells", in IEEE International Symposium on Electromagnetic Compatibility, Washington, 2000.

[9o] Emission and immunity testing in transverse electromagnetic (TEM) waveguides. IEC 61000-4-20, 2010. 


\section{List of publications}

\section{Peer-reviewed journal papers:}

1. R. Vogt-Ardatjew, U. Lundgren, S. F. Romero, and F. Leferink, "On-site radiated emissions measurements in semireverberant environments," IEEE Transactions on Electromagnetic Compatibility, vol. 59, no. 3, 2017.

2. R. Vogt-Ardatjew and A. E. Sowa, "Accurate experimental field mapping in the close vicinity of a pyramidal absorber using the monostatic optically modulated scatterer technique," IEEE Transactions on Electromagnetic Compatibility, vol. 57, no. 6, 2015.

\section{Selected peer-reviewed conference papers:}

1. A. E. Sowa and R. Vogt-Ardatjew, "Evaluation of the loading effect on the optically modulated scatterer," in International Symposium on Electromagnetic Compatibility, EMC EUROPE 2016, Wroclaw, 2016.

2. R. Vogt-Ardatjew and F. Leferink, "Experimental plane wave and random field coupling to uniform and non-uniform transmission lines," in International Symposium on Electromagnetic Compatibility, EMC EUROPE 2015, Dresden, 2015.

3. A. Ubin, R. Vogt-Ardatjew, F. Leferink, M. Zarar, M. Jenu, and S. van de Beek, "Statistical analysis of three different stirrer designs in a reverberation chamber," in Asia-Pacific Symposium on Electromagnetic Compatibility, APEMC 2015, Taipei, 2015.

4. R. Vogt-Ardatjew, A. E. Sowa, and F. Leferink, "The effect of magnetodielectric absorbing coating on unsymmetrical antenna cables," in AsiaPacific Symposium on Electromagnetic Compatibility, APEMC 2015, Taipei, 2015.

5. R. Vogt-Ardatjew, S. van de Beek, and F. Leferink, "Experimental extreme field strength investigation in reverberant enclosures," in International Symposium on Electromagnetic Compatibility, EMC EUROPE 2014, Gothenburg, 2014. 
6. R. Vogt-Ardatjew, S. van de Beek, and F. Leferink, "Influence of reverberation chamber loading on extreme field strength," in International Symposium on Electromagnetic Compatibility, Tokyo, 2014.

7. R. Vogt-Ardatjew and A. E. Sowa, "The influence of the scattering probe on the measurement results of electromagnetic fields by the monostatic modulated scatterer technique," in International Symposium on Electromagnetic Compatibility, Tokyo, 2014.

8. R. Vogt-Ardatjew and F. Leferink, "Observation of maximal and average field values in a reverberation chamber," in International Symposium on Electromagnetic Compatibility, EMC EUROPE 2013, Brugge, 2013.

9. R. Serra, R. Vogt-Ardatjew, and F. Leferink, "Design and performance evaluation of fast electromagnetic field probes," in International Symposium on Electromagnetic Compatibility, EMC EUROPE 2013, Brugge, 2013.

10. R. Vogt-Ardatjew, R. Serra, L. G. Hiltz, and F. Leferink, "Response time of electromagnetic field strength probes," in Asia-Pacific Symposium on Electromagnetic Compatibility, APEMC 2013, Melbourne, 2013.

11. R. Vogt-Ardatjew, S. van de Beek, and F. Leferink, "Wide-band antennas for reverberation chambers," in International Symposium on Electromagnetic Compatibility, EMC EUROPE 2012, Rome, 2012.

12. R. Vogt-Ardatjew and A. E. Sowa, "Experimental field mapping in direct vicinity of a pyramidal absorber using modulated scatterer technique," in International Symposium on Electromagnetic Compatibility, EMC EUROPE 2012, Rome, 2012.

13. S. van de Beek, R. Vogt-Ardatjew, H. Schipper, and F. Leferink, "Vibrating intrinsic reverberation chambers for shielding effectiveness measurements," in International Symposium on Electromagnetic Compatibility, EMC EUROPE 2012, Rome, 2012. 